
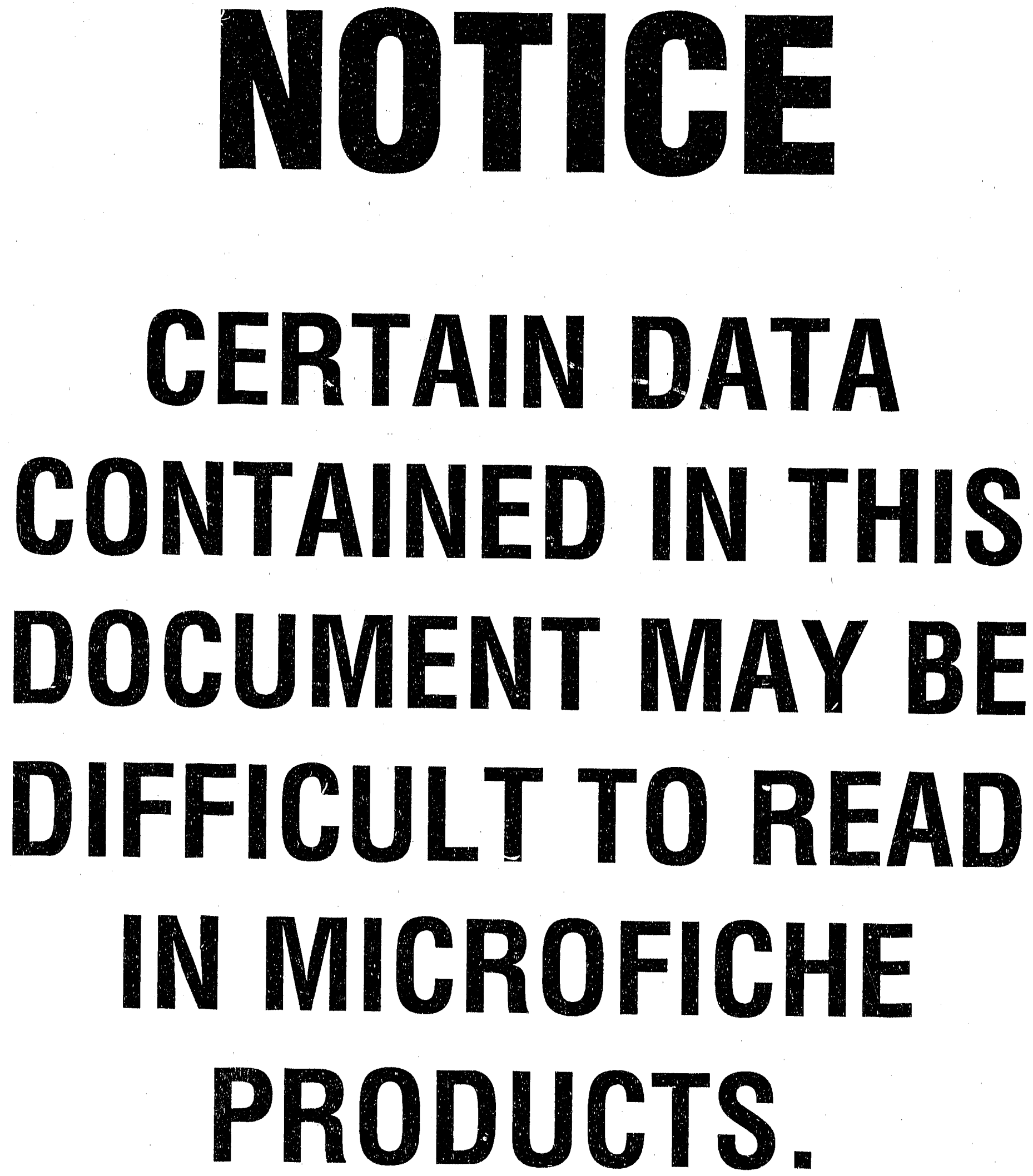
UCRL-ID- -103856

DE91 002542

\title{
An Interactive Program for the Analysis and Design of Advanced Laser Targets*
}

\author{
Robert Thoe \\ Lawrence Livermore National Laboratory \\ Livermore, CA 94550 \\ University of California
}

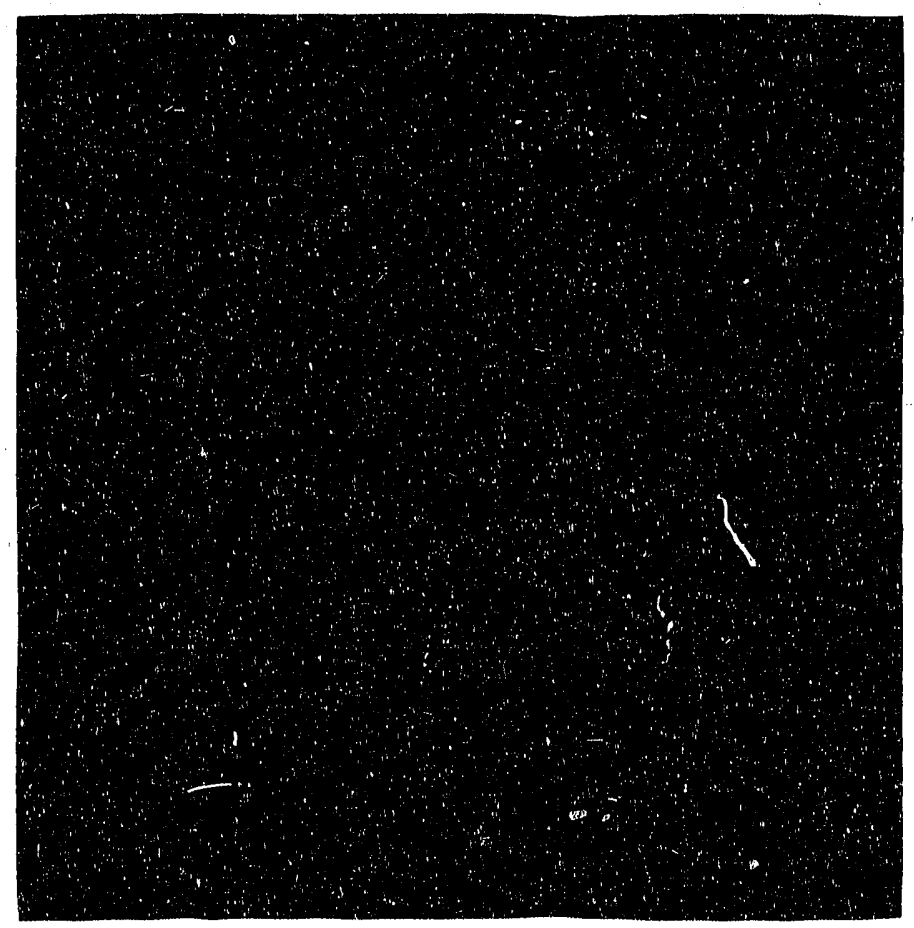

*This work was performed under the auspices of the U.S. Department of Energy by Lawrence Livermore National Laboratory under contract No. W-7405-ENG-48. 


\section{Introduction}

Experiments are currently in progress testing the optical quality of cryogenic targets made using the $\beta$-layering process. This process uses the heat liberated from decaying tritium to sublimate thick parts of the DT layer. This sublimated DT is subsequently condensed on a thinner part of the shell thereby tending to equalize the layer thickness. The uniformity and stability of the ice layer formed on the inside of these capsules is of fundamental importance.

The experiments now being performed simply photograph a capsule through a pair of transparent windows in the dewar as the temperature is lowered below the triple point and finally the sublimation point of the DT mixture. The analysis of these photographs is made difficult by the strong refraction and multiple reflections of the light by the sapphire shell (index of refraction 1.8) and the relatively weak refraction at the vacuum solid DT interface (index of refraction 1.1). Photographs thus far obtained show very little difference between an empty shell and a shell which is believed to be uniformly layered with hydrogen ice. The difficulty in interpreting these data has led to the establishment of an effort to simulate these measurements by direct computational methods.

The code which is the subject of this report is a simple ray tracing routine which allows the operator the freedom to encer as many as 50 different objects - light sources, opaque solids or surfaces, or transparent objects, placed anywhere in space. The program is written in Absoft Fortran for the Amiga, but the source code (except for the graphics subroutines) should compile without any major changes and run on any Macintosh.

The next section gives a brief outline of how the calculation proceeds. This is followed by a presentation of some of the results obtained and a description of some additional features being implemented for future use. A listing of the source deck is added as an appendix. This source can be downloaded from the LEAF where it resides in my file space.

\section{How it Works and What it Does}

The code is divided into three main sections.

1) Geometry definition and bookkeeping 
2) Transport

3) Calculation of polarization and intensity

The geometry can be defined interactively or through a user supplied input file. In either case, the user must specify the size of the picture to be generated in pixels. The screen on the Amiga made the maximum size of $300 \times 500$ convenient, but larger arrays may be specified if the corresponding dimension is increased. This part was made interactive so that the user may obtain a quick low resolution look at the calculation. Next the user must supply the actual format of the picture and the distance from the origin along the $\mathrm{z}$ - axis. For example, the pictures I had made at TID as a check on these calculations were taken with the target $450 \mathrm{~mm}$ from the camera. Since the shell is about $1.6 \mathrm{~cm}$ in diameter, a rectangular screen $20 \times 20 \mathrm{~mm}$ at $450 \mathrm{~mm}$ was used in these simulations.

Next the code queries as to the polarization of the light source and/or detector. Finally, the user is requested to input a file name for determining the objects. Alternatively, the user may input objects at the console.

Objects are specified as a ten element vector. The meaning of each of the ten elements is dependent on the specific object: but, for all objects, the first eight elements specify the geometry, the ninth element is tine relative (outside relative to interior) index of refraction, and the tenth is the brightness (for sources).

With the objects specified, the calculation cornmences. The calculation proceeds with the execution of a pair of nested do loops starting two statements above the statement labeled 97. Initially, the code calculates the direction cosines for a given pixel for a ray starting at the origin. For polarized light the code also calculates the correct polarization for this ray.

Control is then passed to subroutine transport to see which object, if any, the ray intercepts first. This subroutine solves analytically for the intersection of the ray witis each object and checks to see which has the shortest path. It then checks to see if the object is a source. If it is, it returns to the main program and adds the intensity to the intensity of that pixel. If the object was not a source, the new direction cosines of both the refracted and reflected ray are calculated along with the angle of incidence and angle of refraction and passed back to the main program. In the main program, if the object was not a source or a sink, the subroutine fresnel is called to calculate the intensities of the refracted and reflected rays. 
Fresnel calculates the reflectivity and the transmission by calculating the fresnel coefficients and squaring them. In order to accomplish this, the $s$ and $p$ polarization directions must first be determined. Once these directions are determined, the $s$ and $p$ intensity is determined and hence the amount of $s$ and $p$ reflected and refracted intensity are calculated. These intensities are all determined for incoherent light.

After fresnel, the intensities of the refracted and reflected beams are check, and a decision is made. If either reflected or refracted ray is less than .001 its original intensity, that beam is dropped. If both are still brighter than this limit, a pointer is incremented so that the refracted ray will be traced at a later time. Then the reflected ray is sent to transport and the process is repeated until the reflected intensity is weaker than the above limit or the reflected ray hits a source. At this point control is passed to statement label 98. This part of the program picks up the next refracted ray and sends it through transport. As it does, the code still follows only the reflected rays but keeps track of any of the refracted rays that are sufficiently bright. Eventually, the intensity of all the rays that don't hit a source or sink will reach an intensity level lower then .001 . If this doesn't happen before the index reaches 200, the loop is automatically terminated.

Finally, if a trace flag were set, a file is opened which keeps track of all the reflected and refracted rays and a graphics window is opened that plots these rays as viewed in the $x-z$ plane. This routine is for diagnostic purposes.

\section{Resivite}

Figures 1 - 3 show the results of three measurements and the corresponding calculations. The measurements were taken at a studio at TID using an $f / 194 \times 5$ camera with $t_{\max } 1000$ film. Unfortunately, the wedge made for these exposures was considerably over exposed and useless for calibration purposes. Therefore, wedges from NSCS were used to get exposure levels. The three figures were made with different sized backlighters. In each case, the distance from camera to target was $45 \mathrm{~cm}$ and the backlighter was $8.5 \mathrm{~cm}$ behind the target. The backlighter consisted of a black piece of cardboard with a circular aperture cut into it. Behind this cardboard was placed a white sheet of paper which was 
illuminated with a studio light. Considerable care was taken in order to keep the backlighter as flat as possible.

The calculations were made with a resolution of $300 \times 300$ pixels. The photographs were digitized with a $12 \mu \mathrm{m}$ square slit. The calculated image was sent $o$ the LEAF where it was stretched (IDL congrid) to the same size as the digitized photograph. The digitized photograph had been adjusted in exposure so that the background was at the same intensity $a s$ the calculated image. No other normalizations were done.

The first three figures show not only these images, but also horizontal lineouts at corresponding locations. The only difference in these three pictures is the size of the backlighter - 25, 50, and $100 \mathrm{~mm}$ diameter for Figs. 1 - 3, respectively. Inspections of these images reveal reasonable agreement between calculations and data - especially in the limb of the target. Many of the features in the photographs appear to be reproduced extremely well by the calculation. A discrepancy between calculation and data exists primarily in the region of the sphere directly inside the dark band. The photographs show a smooth increase in intensity from the dark band to the bright center. The calculations show a region of increased brightness due to some reflections between the inner wall and the outer wall followed by a noticeable decrease in intensity and finally a monotonic increase in intensity to a maximum at the center. The sudden decrease in intensity corresponds to imaging the edge of the backlighter. A trace of this region was done to show that this is indeed the case. The results of this trace are presented in Figs. 4 and 5. These two figures show the rays as seen in the $x-z$ plane, and represent two rays that are separated by only a single pixel. Appendix $B$ is a printout of the positions and intensities of each ray at each surface. It is not known at this time why the data disagree so strongly with the calculation. It may be the result of nonspecular reflection smoothing out the sharp image, but this is pure speculation. It would point out that I assembled these targets myself and did not do a very good job. The hemishells were adhered to each other with optical grease and fingerprints were deposited on both the inside and outside surfaces.

Several other calculations were done. In order to try to optimize the measurements and determine how much anisotropy could be detected, the calculations shown in Figs. $6-11$ were done. Figu. \& 6 is a lineout of a sapphire shell which a $1 \mathrm{~mm}$ thick layer of hydrogen ice inside it. The shell 
is $375 \mathrm{~mm}$ from the origin and the backlighter is at $410 \mathrm{~mm}$. The diameter that the backlighter is $50 \mathrm{~mm}$. Figures 7 and 8 are similar to Fig. 6 except that the backlighter is $20 \mathrm{~mm}$ and $250 \mathrm{~mm}$ in diameter, respectively. Figures 9 and 10 are lineouts of a geometry identical to Fig. 6 except that the ice is geometry similar to Fig. 7 and the ice offset by $250 \mu \mathrm{m}$.

Finally Figs. 12 and 13 are calculations for geometry similar to that of Fig. 6, except that the sapphire was replaced by quartz $(n=1.56)$ and LiF ( $n$ 1.42), respectively. These calculations lead me to believe that probably the sapphire gives the best contrast for observing the DT ice layer and that irregularities as small as 100 should be very easy to spot.

\section{Conclusions}

A code capable of synthesizing the images obtained from backlighting transparent shells has been written and appears to $e$ in moderate agreement with actual photographs. This code, however, neglects birefringence and has no method of introducing scattering. Subroutines are currently being written which will allow the introduction of different geometries-cylinders and prisms with spherical voids which may offer better diagnostics for the $\beta$-layering process. 


\section{Eiraune Captions}

1-3) Photographs and simulations of an empty sapphire shell backlight withis a 1050 and 50 mm diameter backlight. The shell-camera distance was $45.0 \mathrm{~cm}$ and the camera-backlight distance was $53.5 \mathrm{~cm}$.

4) "Trace" of the region around coll. 103, i.e., $r=3 \mathrm{~mm}$. This figure shows how each ray branches into reflected and refracted components. In this figure, the light source is off the edge of the plot so rays that would have intercepted it were not plotted.

5a) Traces similar to Fig. 4 but with a much expanded scale. Two rays are shown intercepting the source.

5b) Same as Fig. 5a, except the incident ray is shifted by one pixel. Now only one ray hits the source. A complete printout of this trace is in Appendix B.

6) Lineout of a full sapphire shell. Index of refraction $n=1.8,65<r<7.6$, $=1.1,5.35<\mathrm{r}<6.35, \mathrm{n}=1, \mathrm{r}<5.35$. The scale is $8 \mathrm{~mm}=500$ pixels. Backlighter is a disk $\mathrm{r}=50 \mathrm{~mm}$ at $\mathrm{z}=410$, Shell is $375 \mathrm{~mm}$ from argon.

7) Similar to Fig. 6 except backlighter radius is $20 \mathrm{~mm}$.

8) Similar to Fig. 6 except backlighter radius is $250 \mathrm{~mm}$.

9) Similar to Fig. 6 except DT $(n=1.1)$ is centered at $z=410, y=0, x=0.1$ m m.

10) Similar to Fig. 6 except DT is centered at $z=410, y=0, x=0.25 \mathrm{~mm}$.

11) Similar to Fig. 7 except DT is centered at $z=410, y=0, z=0.25 \mathrm{~mm}$.

12) Similar to Fig. 6 except that quartz $(n=1.56)$ was used instead of sapphire.

13) Similar to Fig. 6 except that $\operatorname{LiF}(n=1.42)$ was used instead of sapphire. 


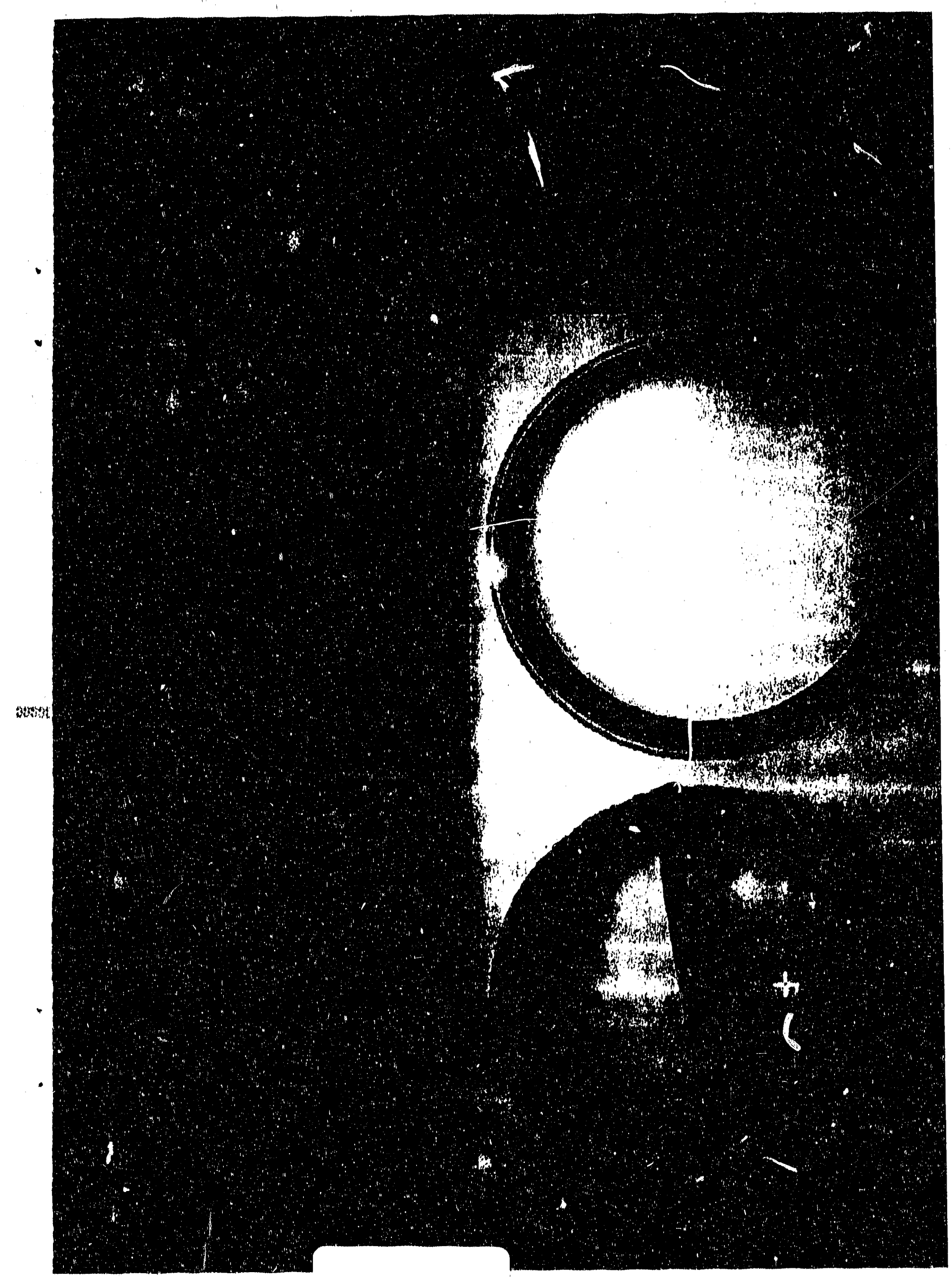

Figure 2 


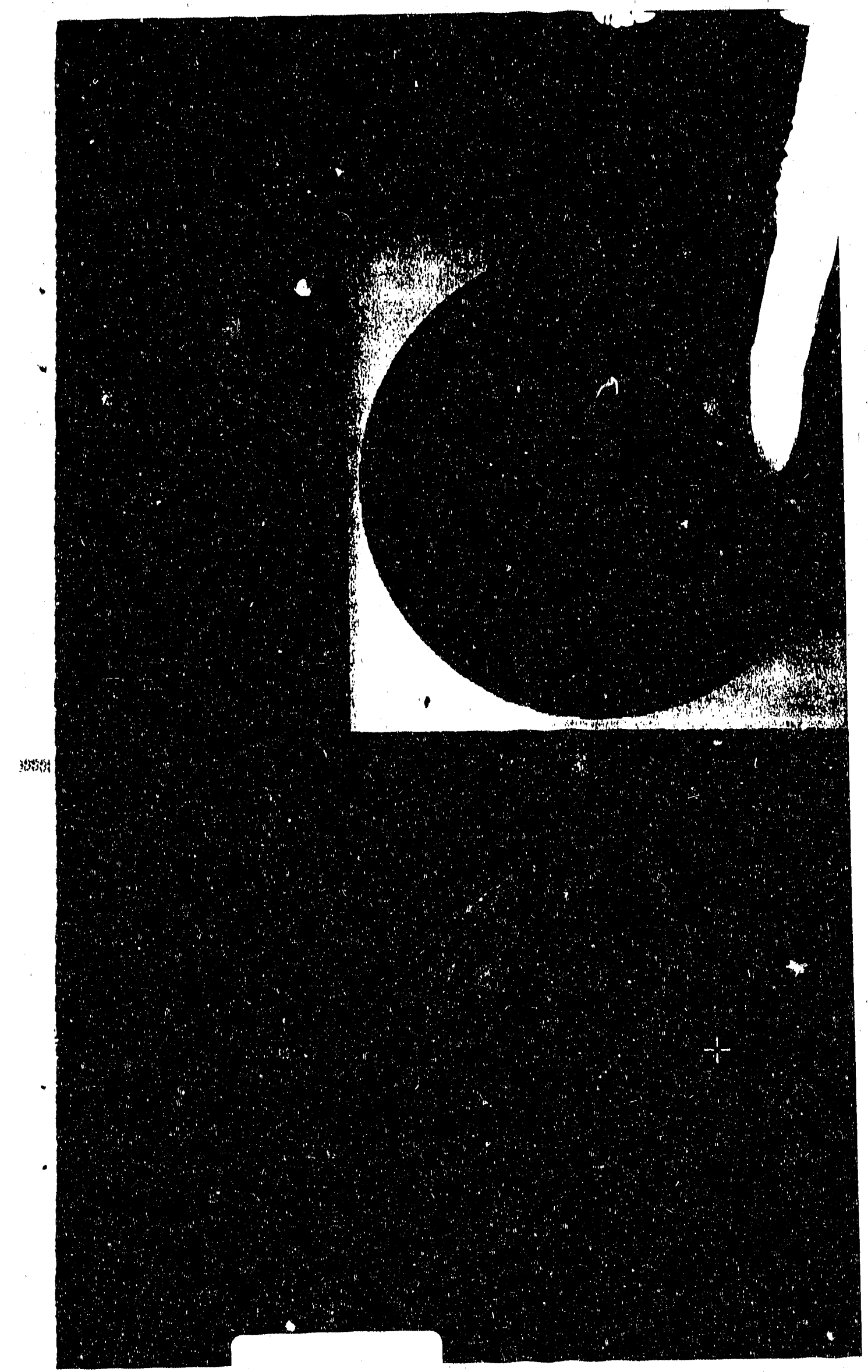

Figure 3 


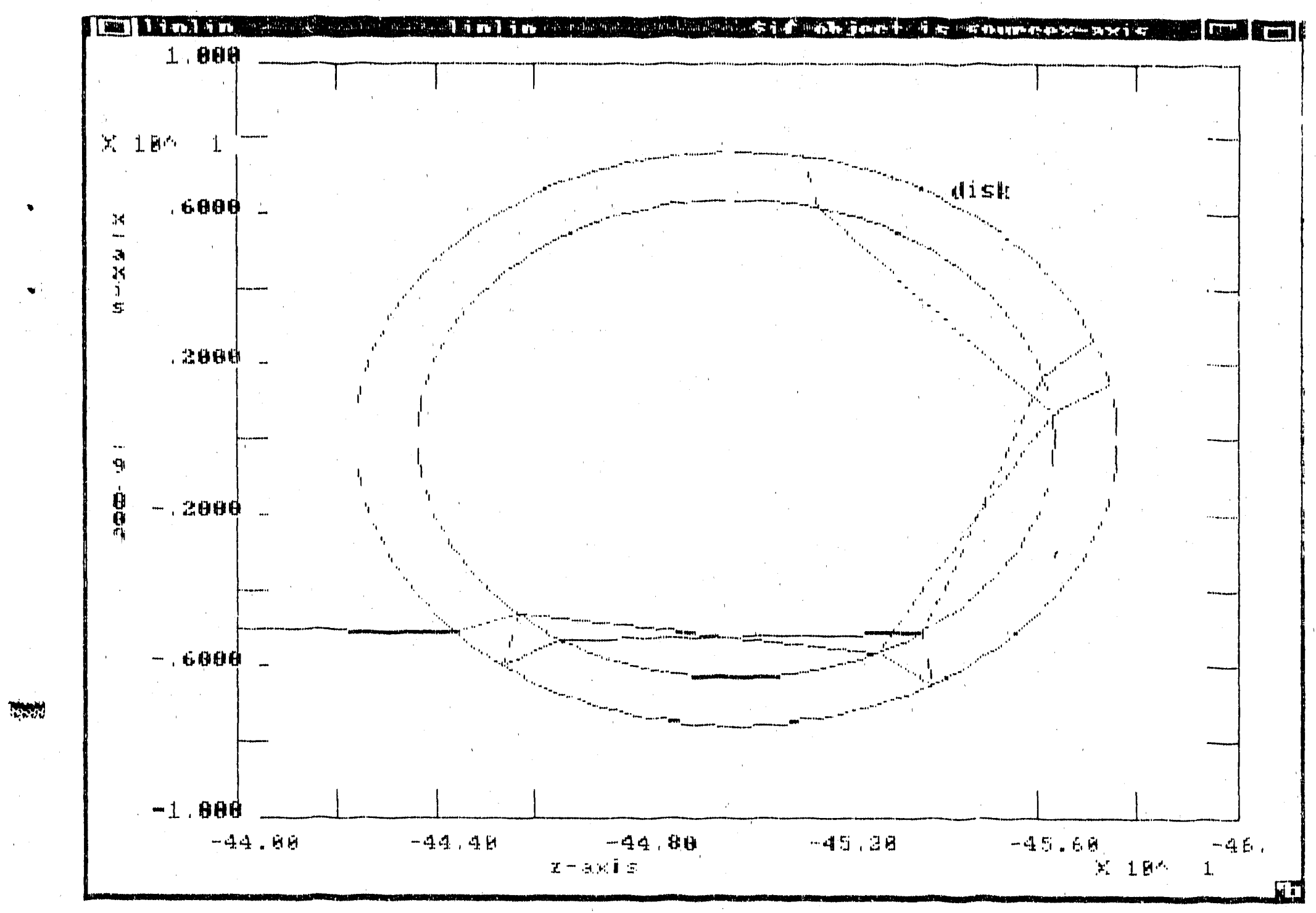

Figure 4 


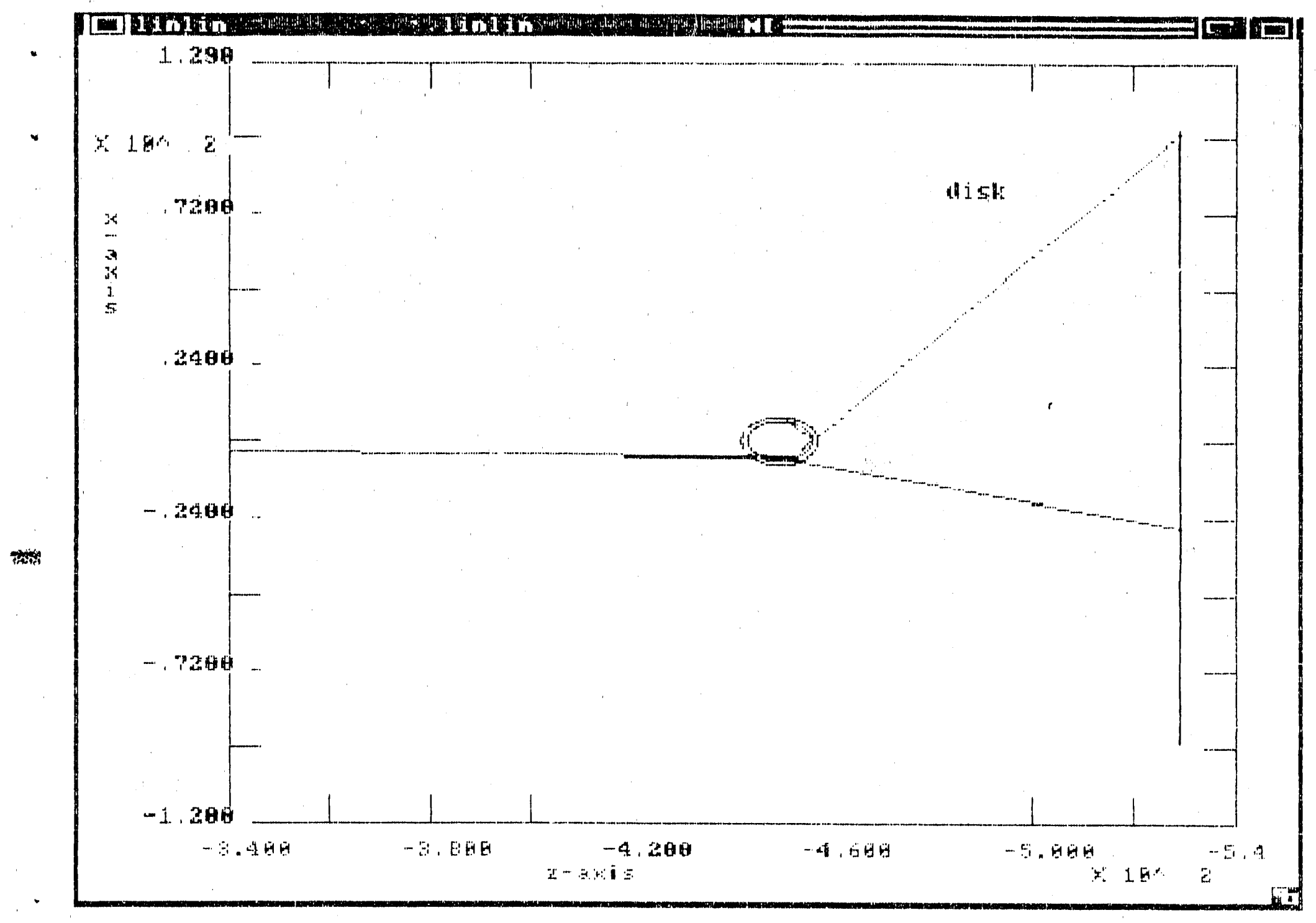

Figure 5a 


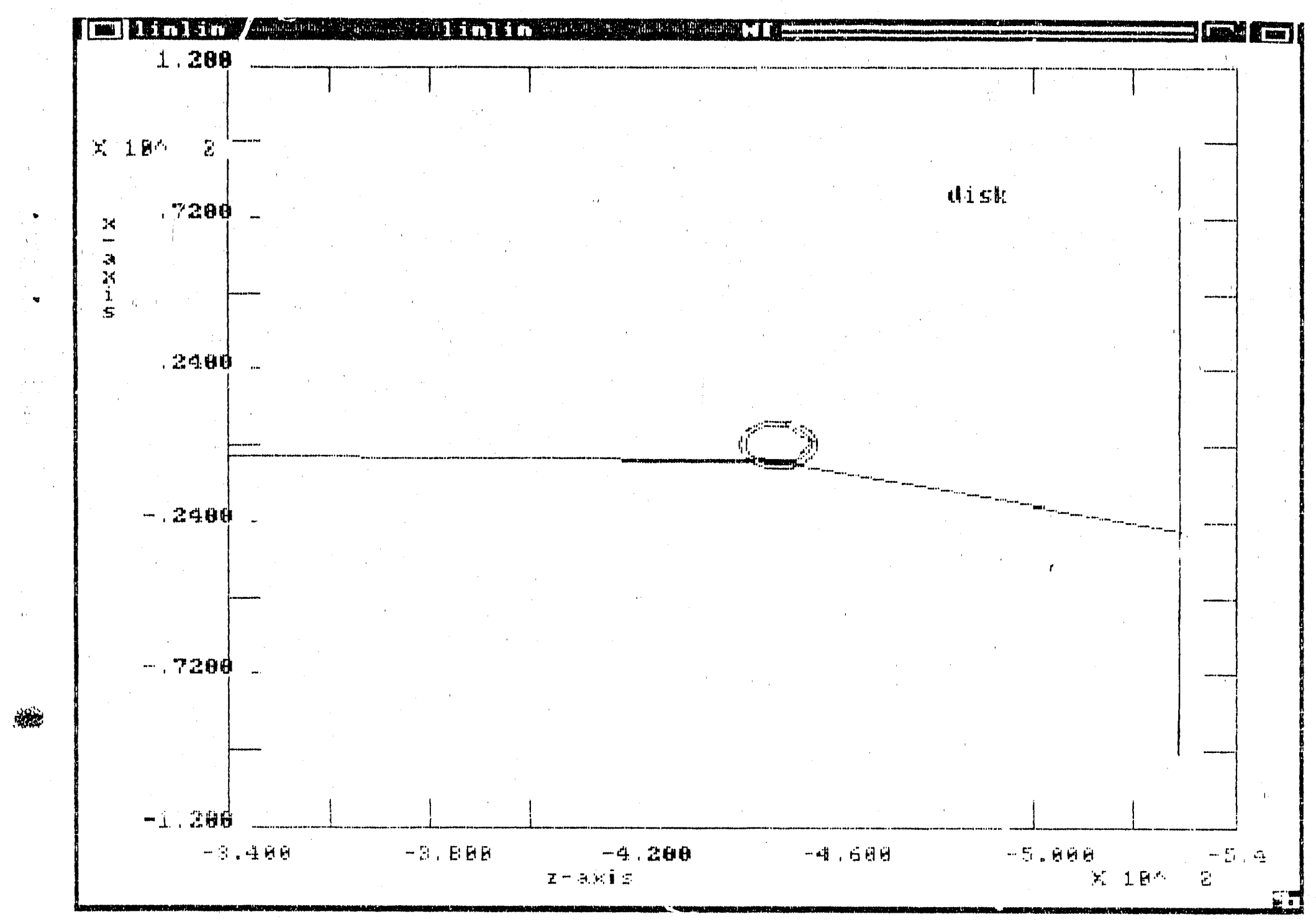

Figure $5 b$ 


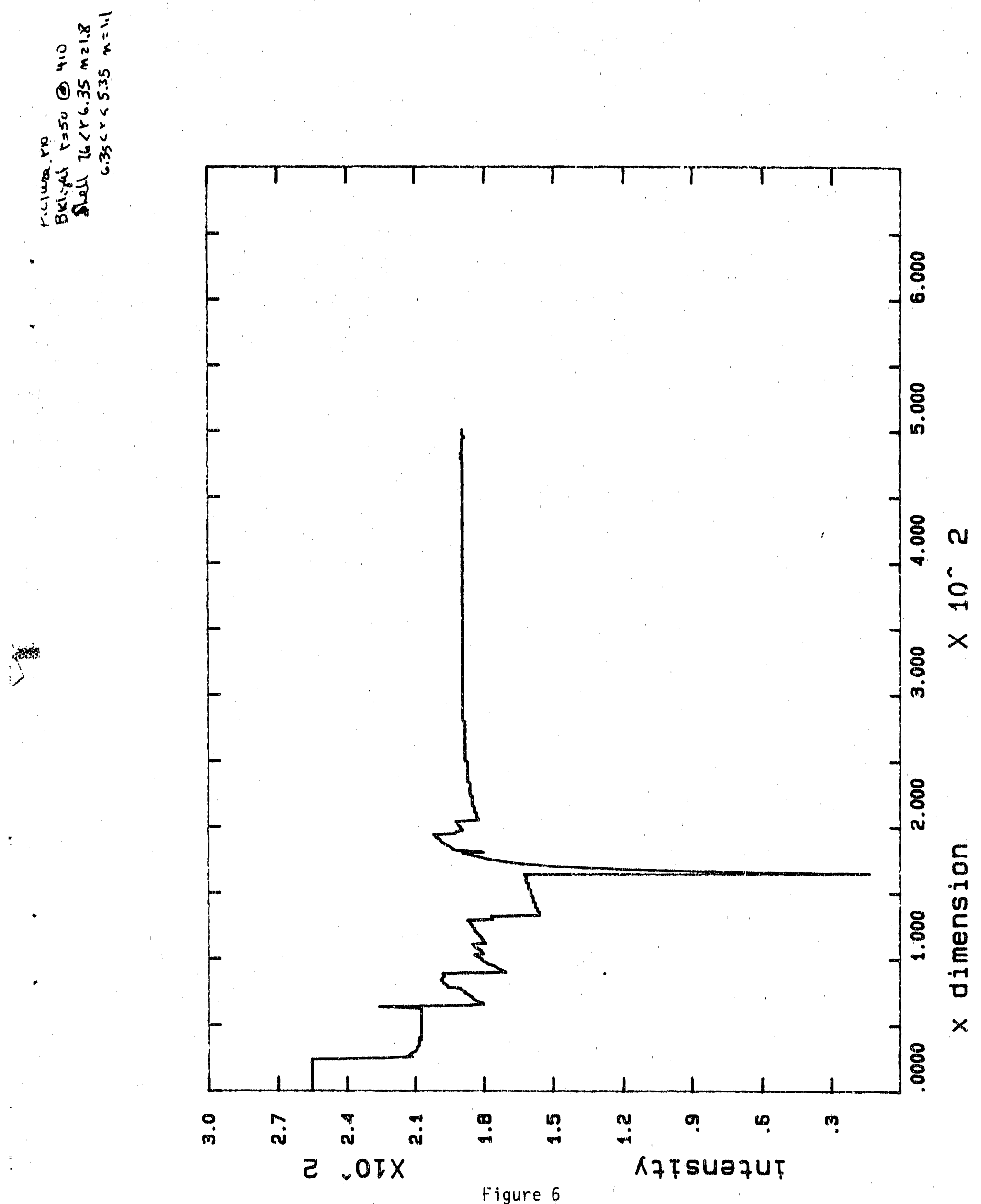




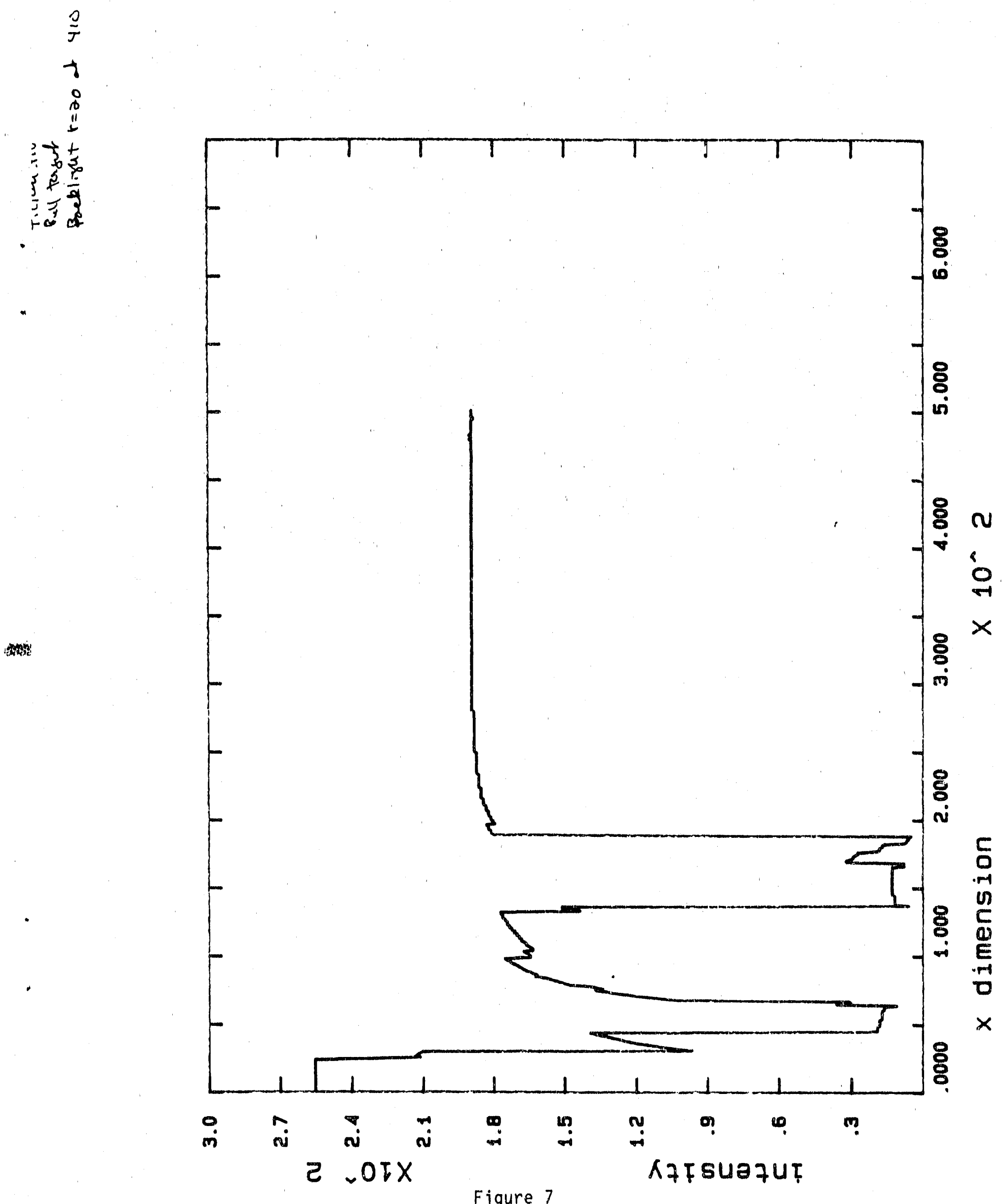

Figure 7 


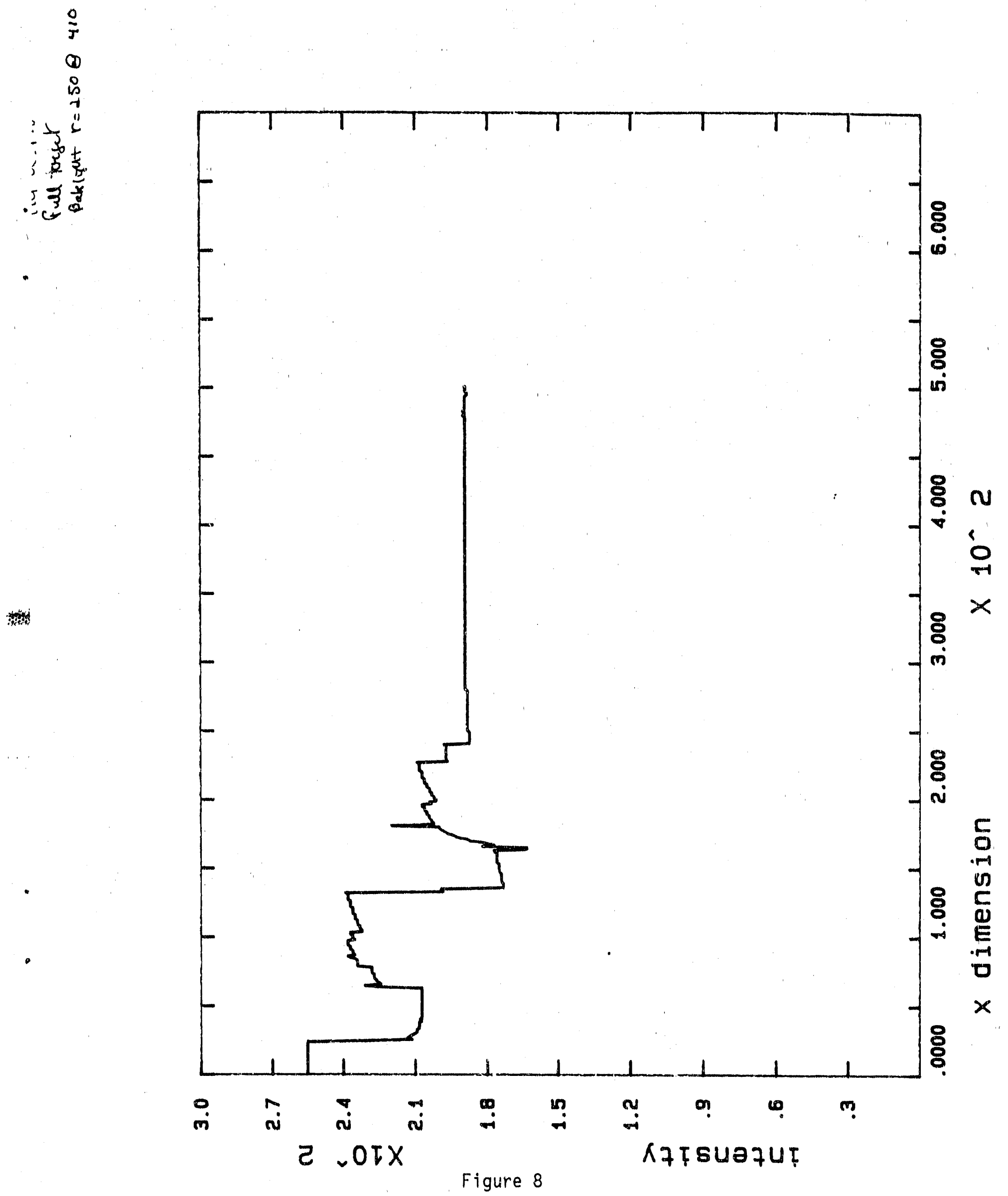


3
0
0

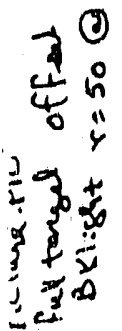

was

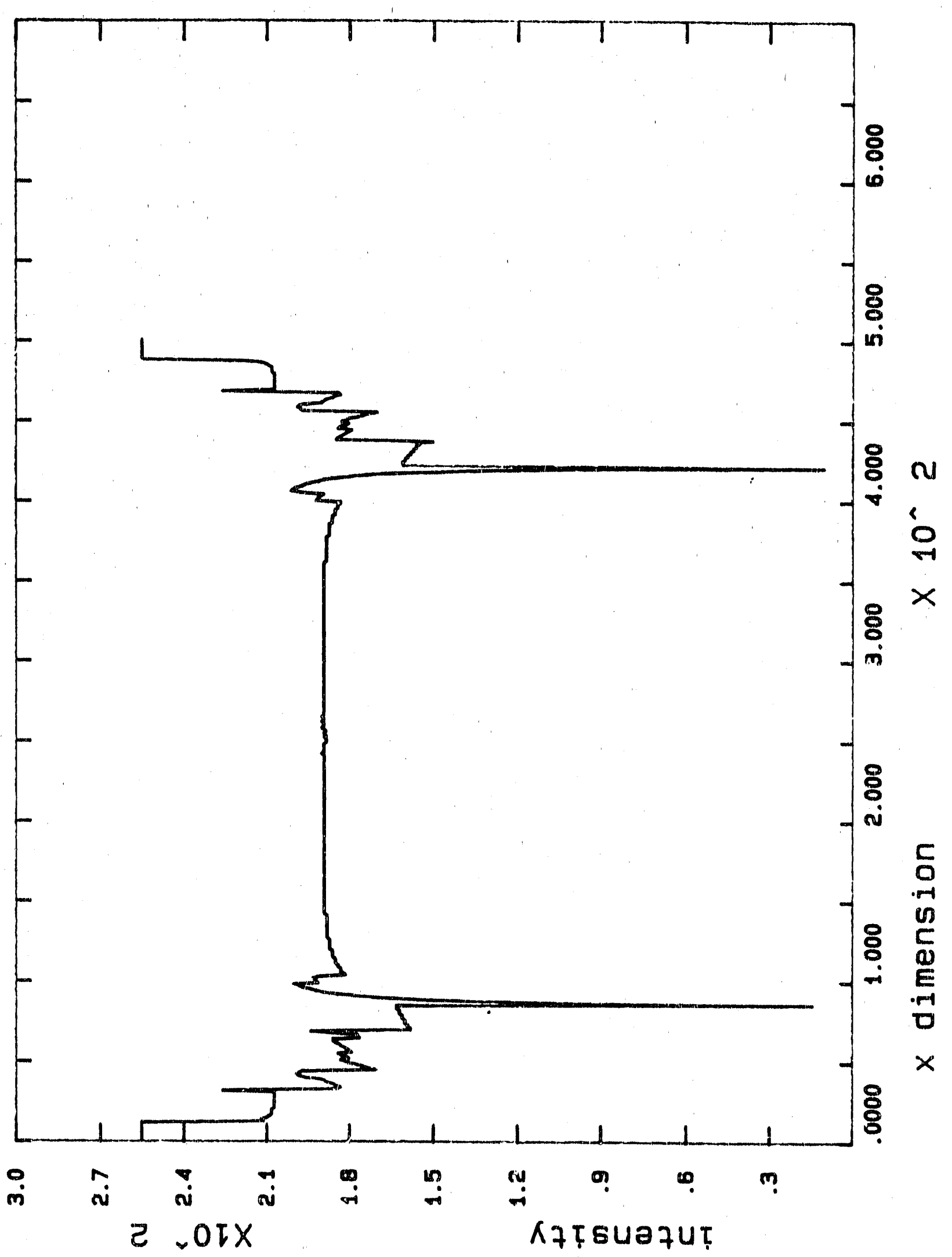

Figure 9 


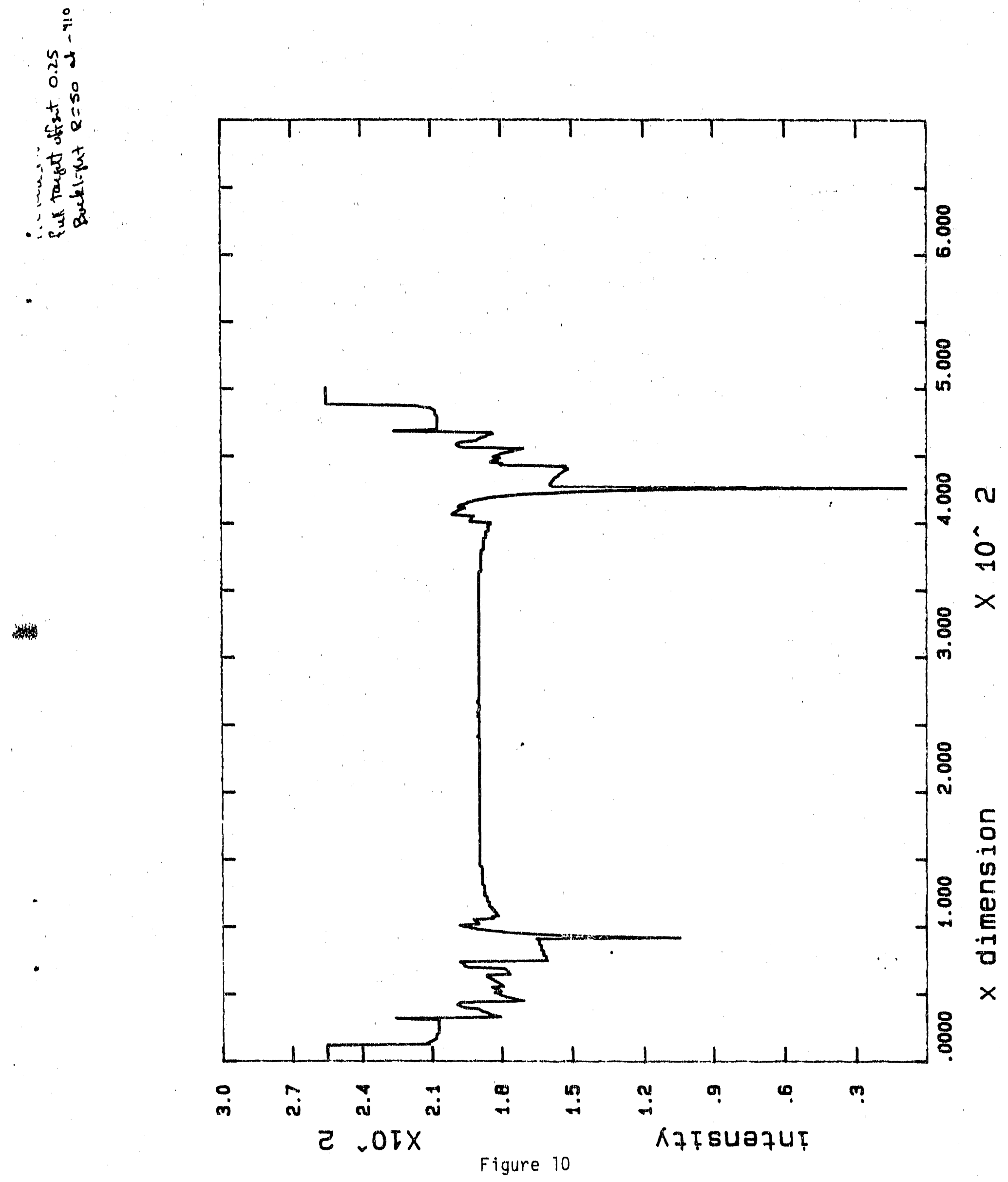




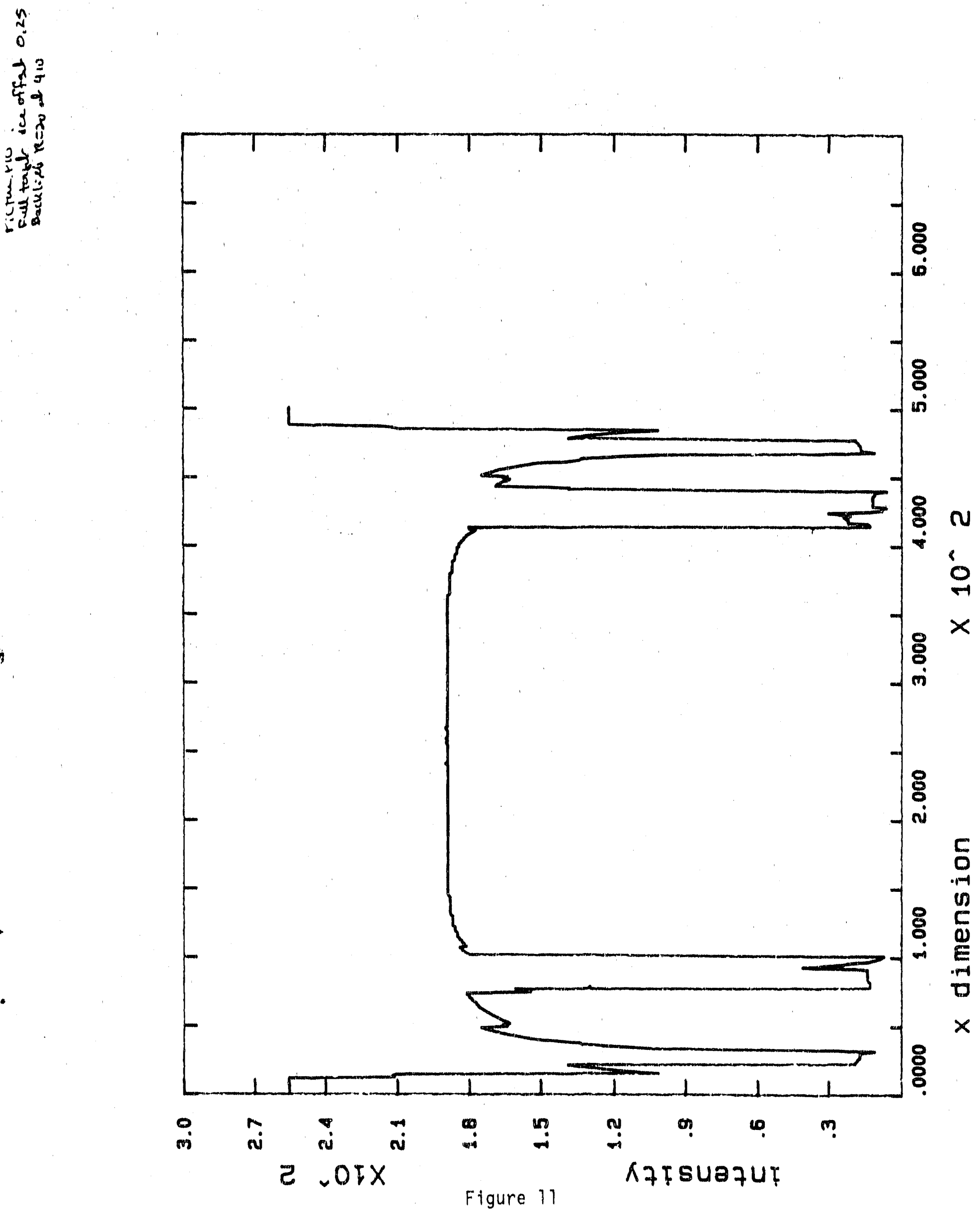




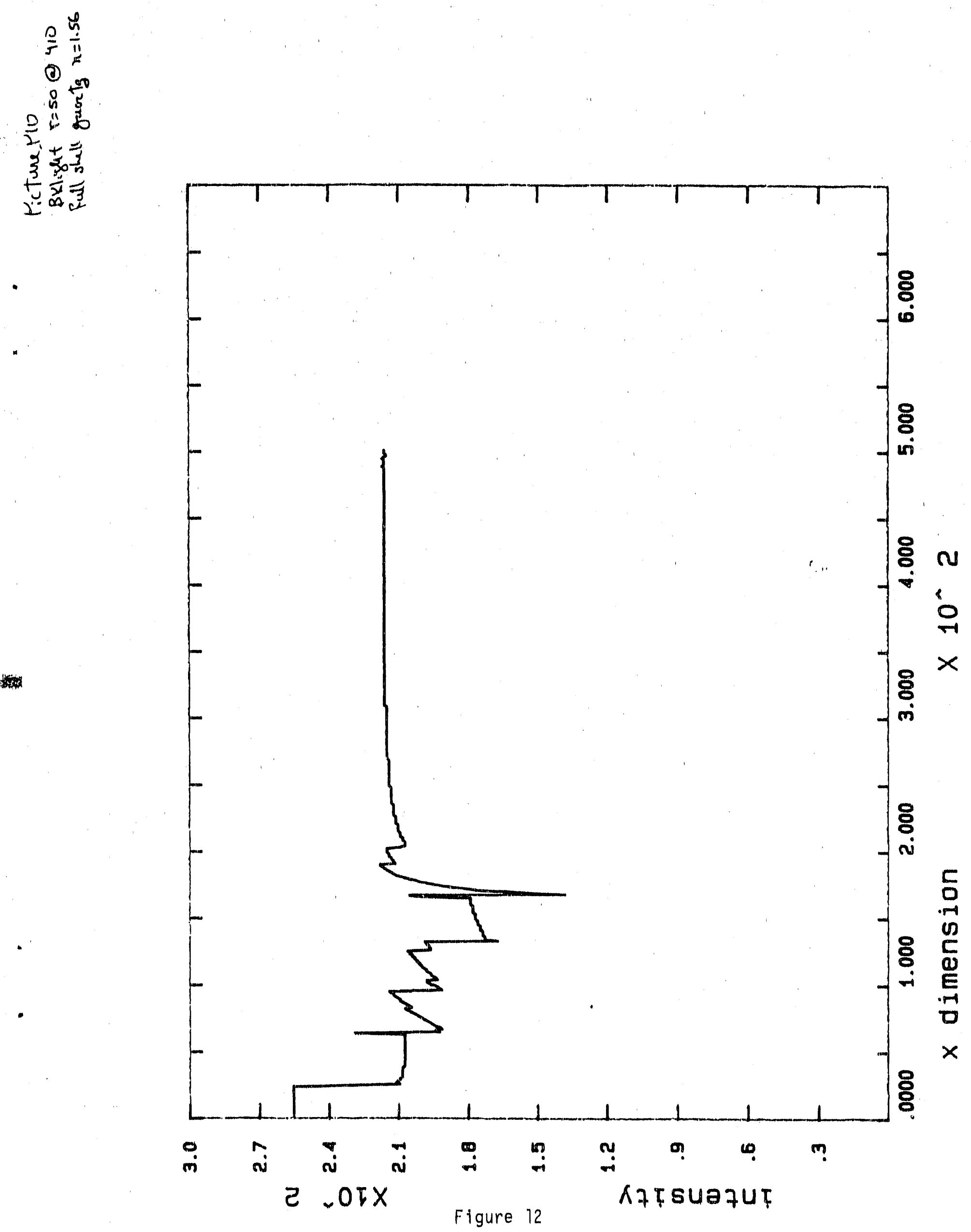




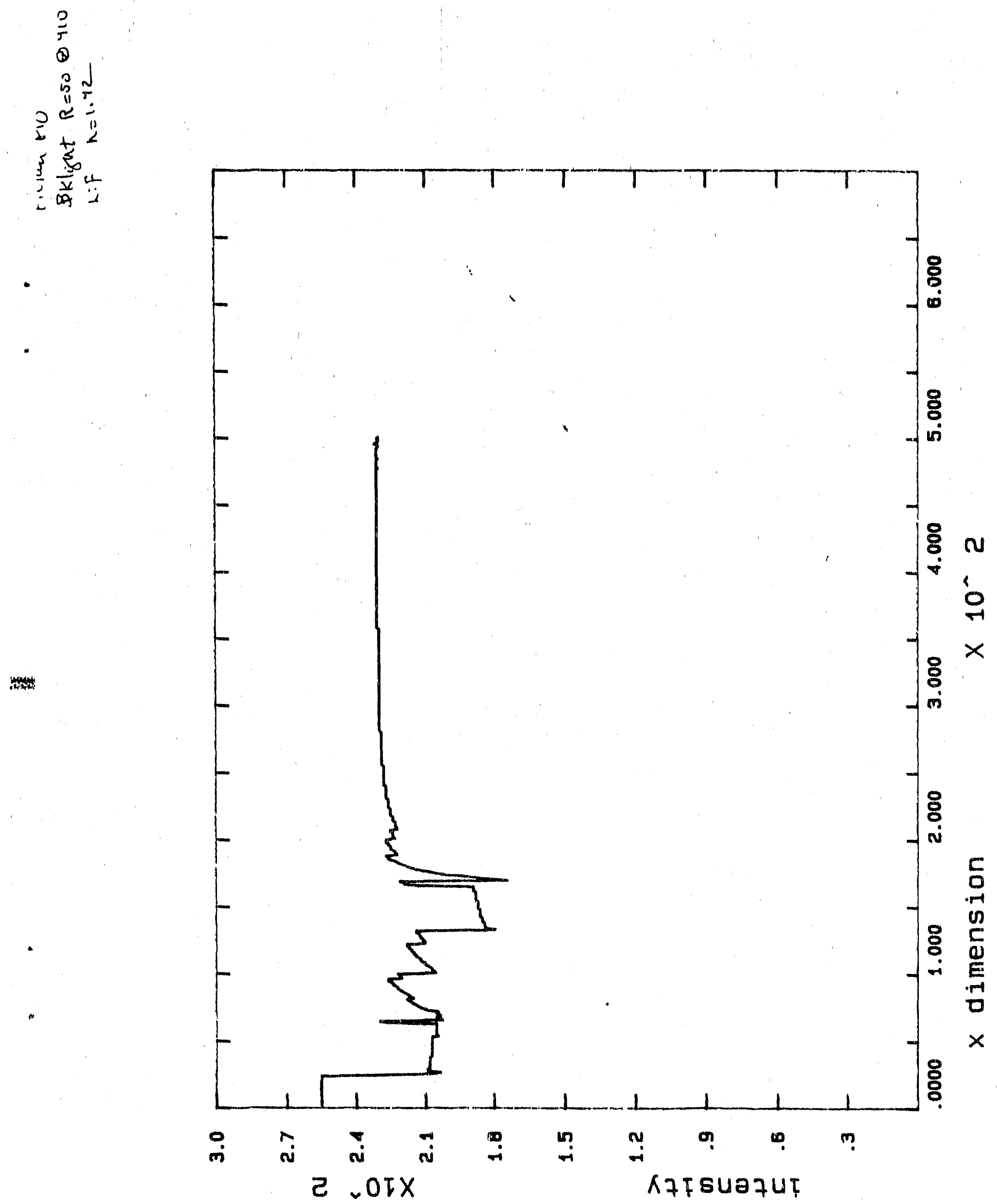

Figure 13 


\section{Appendtx A}

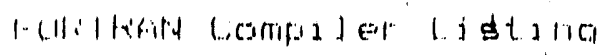

$1.1 .14,4,4,1)$

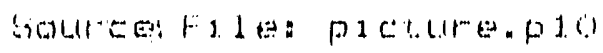

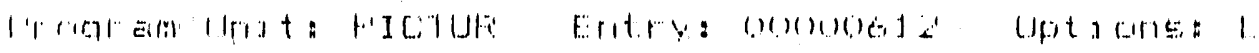

$1111111 \% 1$

$(31) 19.19 .9$

(.11)11.1.19

(11, 11) 14

- 1111111.18

(1).11.1060

(2) $1010 \%$

1) 1000

()oot)

i.

(x) 11.) 1

(4)

(16)1]

$1,(10) 14$

(1)11:

(1)

(m) $1 \%$

(1). (1) 1

(11)

(1) (1) (1)

(i)ist

(1)

(1)

(1) 1124

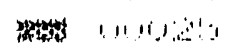

1.)1162

व111)

(11)

$11111,1.1 .1 \%$

11111,41

11111131

(19)10.

1.1010 .5

1 1. 19.91

(.111.) $\therefore 1$.

14,900

$11111, \%$

$+$

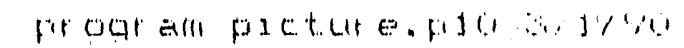

progaram partate

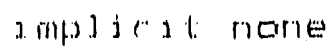

inteqer+l $1(512, \because 00)$

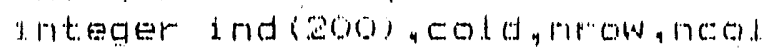

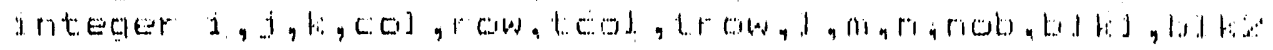

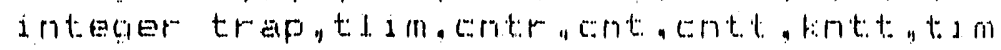

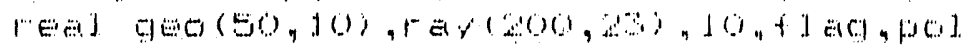

real temp, so

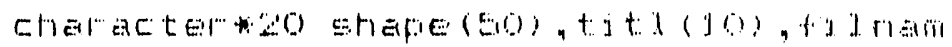

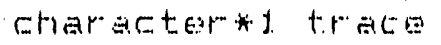

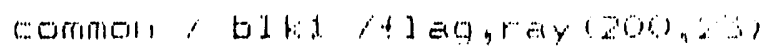

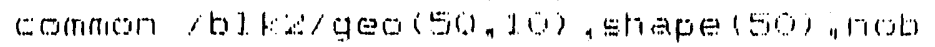

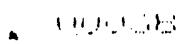

1311,365

in) 1404

iir 11,141

- (11)14:

$1111.21:$

i1. $11 .+4.4$

1114146

$\therefore 11.46$.

(1)i14)

1111.48

1111149

1110.619

(111) 1

(1)

(1)(1)

11111,4

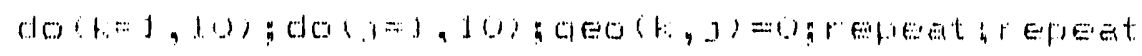

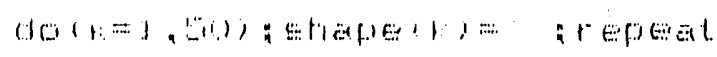

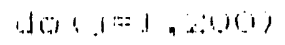

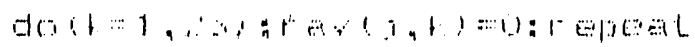

Patrit

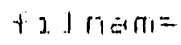

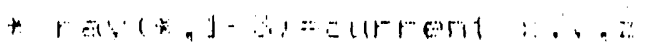

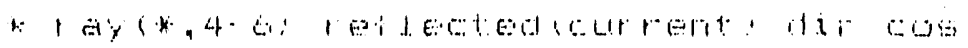

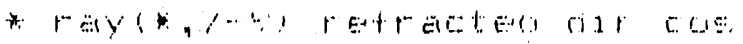

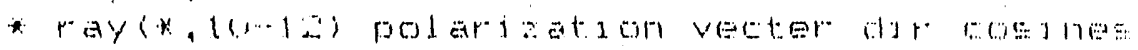

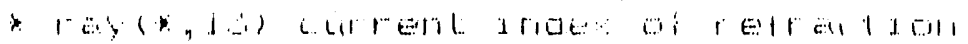

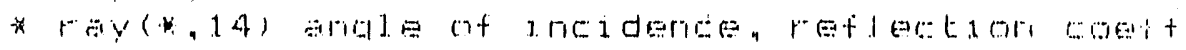

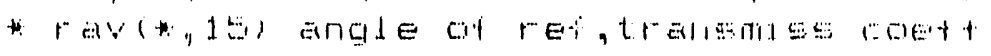

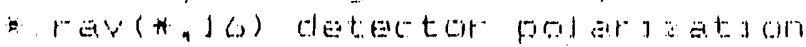

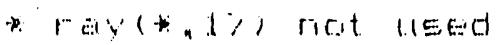

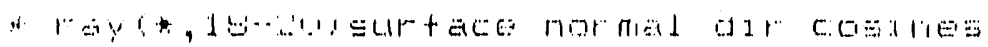

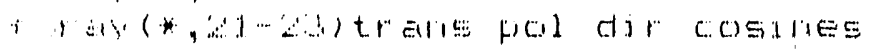

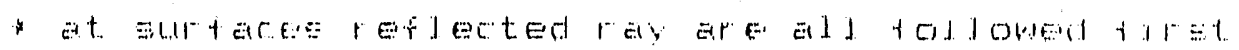

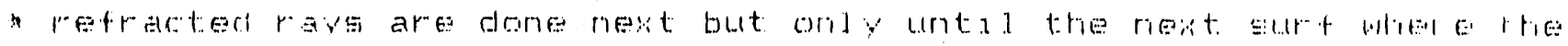

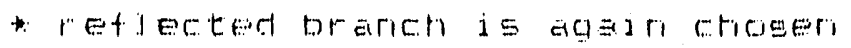

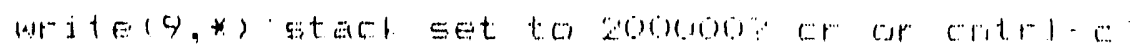

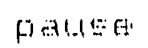

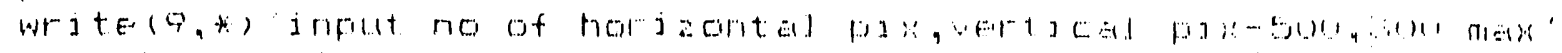

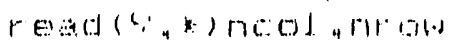




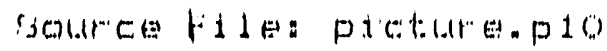

(1) 1.110.19\% (c) $10005 \%$ (ii) 11,100 $(10) 61$

$1(10) 62$ (1) (1) 100004 mosin

$-00006$ oore? (1)1060) 00069 (1) (1) $)$ (i) 101 $(14,1) ;$ (1) 00,14 $010.1 \%$ oon 1.0011 ()ow oor \% nocsol (o)

worde nonoss ocolos 4 ootos: inmed bur. or) ooces (a) (1) inder

ane? 1.110.98

(.)191. 94 1010.....

- 1 (19) nent: (1)

- mory

(1)1 110 (N) 101 (1) $)$ (1)10 (1) 104 - i. 1.06 a) 100 b10: 00108 (14)

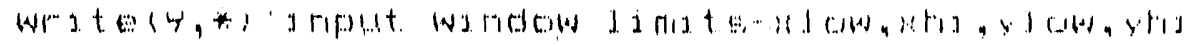
1.

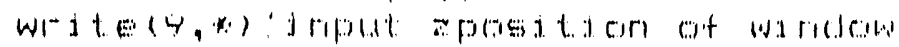
rea

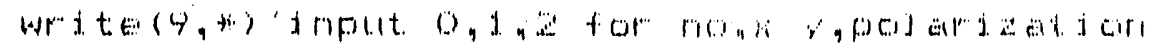
"werd $(i, k)$ pol.

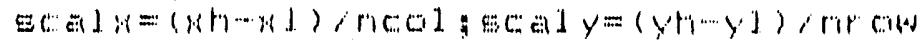

cicale $(16)=0$

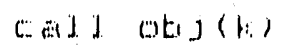

do $(1:=1,1$ not $)$

if (scale(fo). It a abs ( repent:

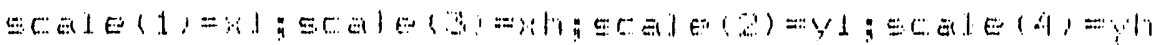

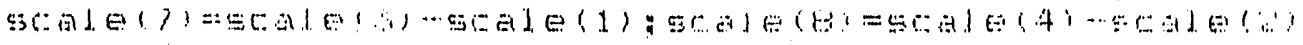

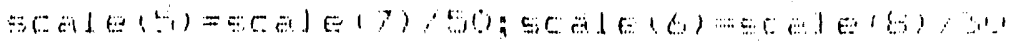

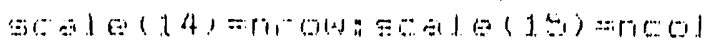

E(c)

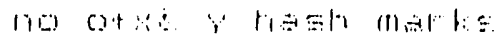

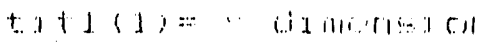

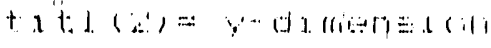

$t+1+1,1=0$

tis.te ot pisetures

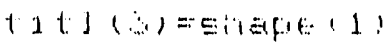

t. i. $1(4)=4.110010$

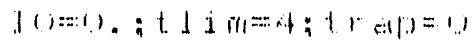

cand tillies (tim)

(a) iter $\operatorname{coc}(\cos \omega)=1$, nrotos:

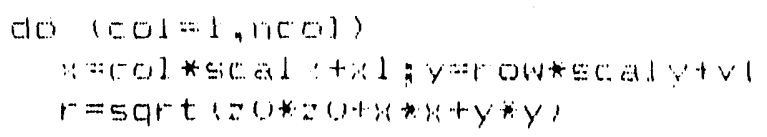


(1). 1910

(.). 111

(1) ] J 2

(1). $11 \div$

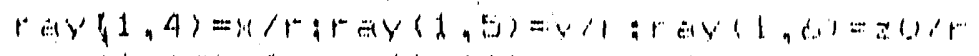

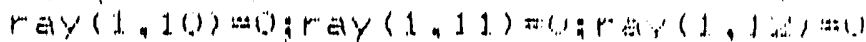

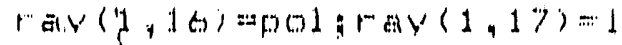

$1+(p())$ : a: 1) thal

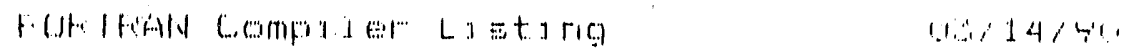

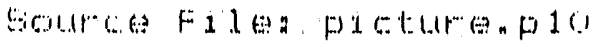

-

(1) 114 (1), 113 () $1 \mathrm{~A}$

- (1) 10

(1) 118

$0011 \%$

(a) 3

(11) $1: 1$

का 11.2

$10] 3$

(1) 1 )

(1) (1) 10.5

(1) 124

(.)10 2

()1120

(0) $12 \%$

$(1,1,1.1,1)$

(1) 101 (i) 3

(ii) $\therefore 4$

1.11

(1) 10

(2) 113

4.11

(16) 45

(1)1) 401

iㅣ सा

1.1.) 4

(1) 14 iा

111 48

$11194 \%$

- 11. 140

1.11 4 ;

$111+124=$

- 1014

$11 ., 191,1$

1)

(1) $13: 3$

$(19,1) \div 3$

$(141) \div 9$

(1). $11.01 \%$

잉a.

(11) 1 ?

14)

(11.)1 91.

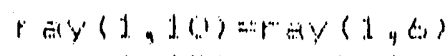

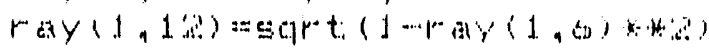

endit

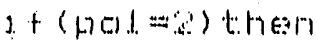

ravil, 1)

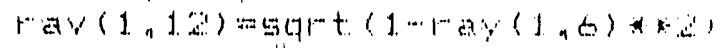

enriclat

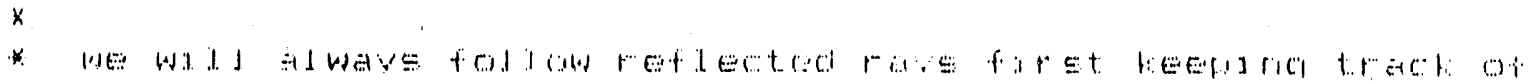

* torarind toed ray

$x$

$*$

$\cot r=1 ; 5 r+1=1 ; \operatorname{lnt} 1=0$

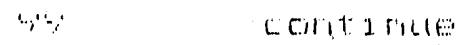

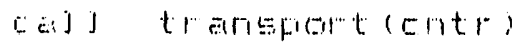

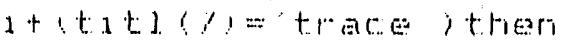

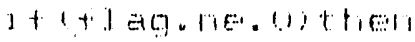

1. to

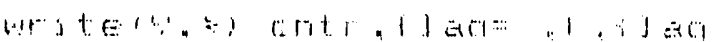

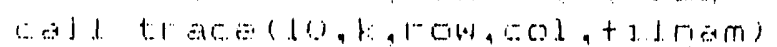

Erida f

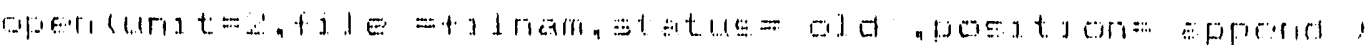

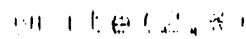

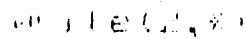

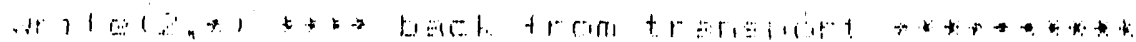

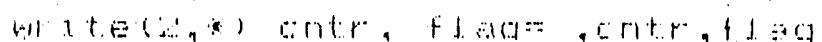

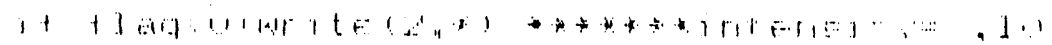

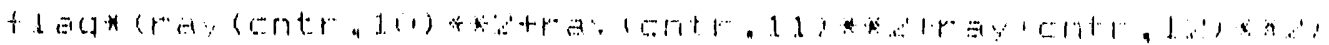

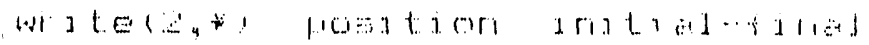

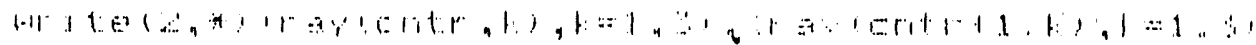

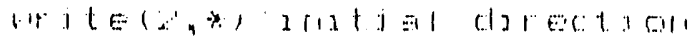

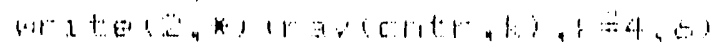

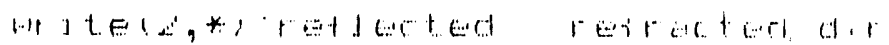

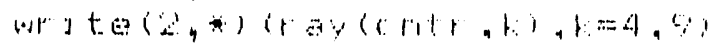

CE1 $1000(20)$

rrad $f$

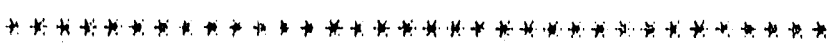

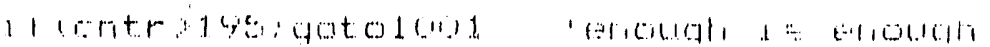




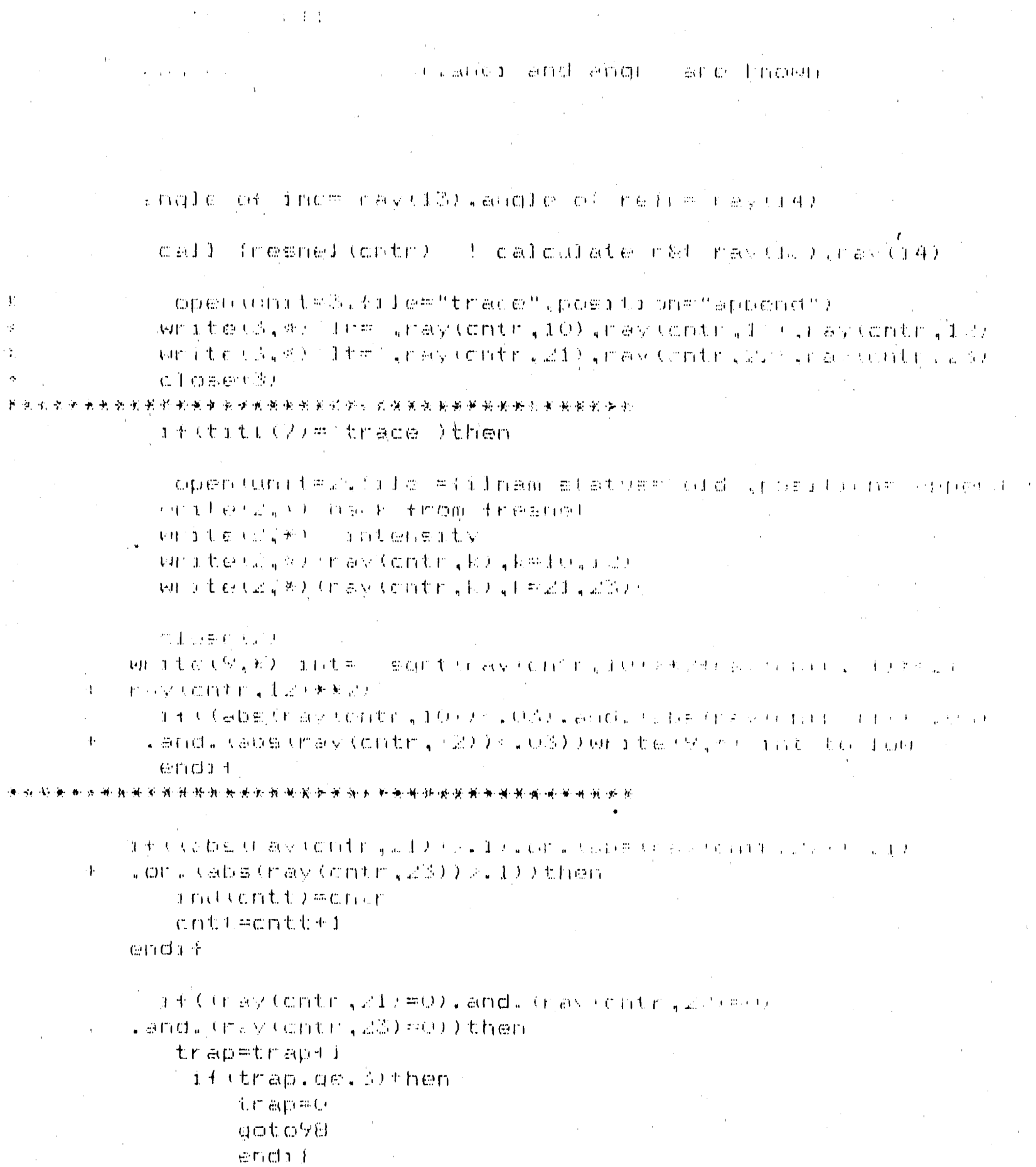


01021

(11)

11026

(19)

(1) 1 (1)

1) 1) ates.

(1)

(1)

(1) 10 s.

\section{else}

trefis

ende f.

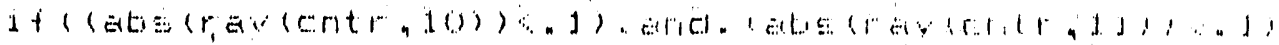

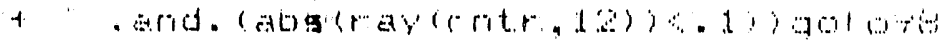

acit 0196

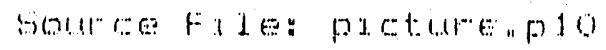

$(1+3)$

- 101

- 1110.00 (102)

1)

101

(1)

(1) 2 का

10 10

(1)

(1)

(1) 19

(i) $)+4$

1)

(1) $2: 44$

(1) 1245

$1044:$

(1) $4 \%$

(1) 24

(1) 44

acteres

(19)

1.11.

(1)

1 10.

1.106.

- 19.14,

$111 \mathrm{~s}: \mathrm{k}$

1. 110

. 1,1019

1901

1) 10101

(1)

1 126

- 21984

111,100

111.

1) 119.

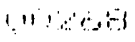

191969

(10): 210

(1) 1

11190

119.5

191294

(1) $2: 5=$

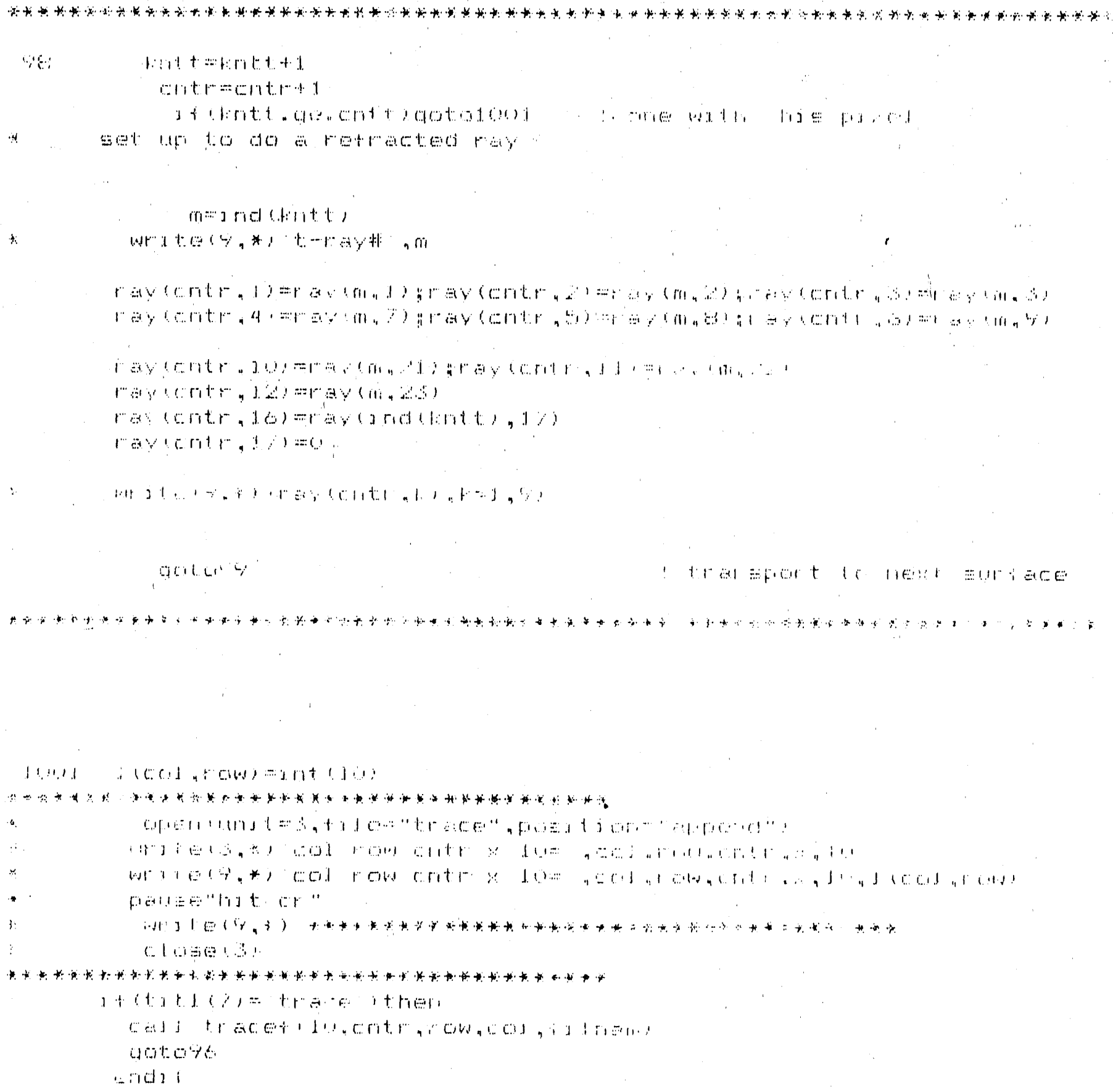


111,1218

(1). 1)

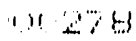

i.11.2\%

. 11020010

110.61

$111928 \%$

11120

i. 10.484

11120

(1) 28 -

- ruteri,
(1) $1.1 \div \cdots 1.1$

t.r. $10=1$

c. rilr $=1$

restat

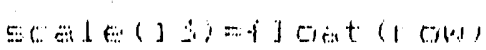

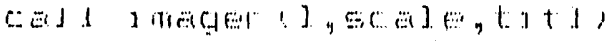

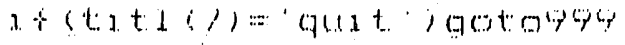

$r e p e$ ent

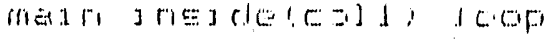

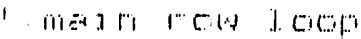

-

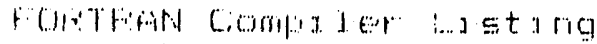

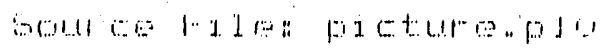

11,48

i 14,8

111,290

110971

ing

1013

(3) 294

ing

तinds

$11119 \%$

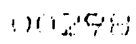

(11) a).11

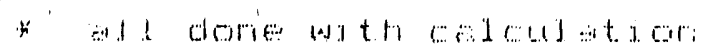

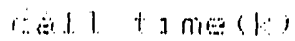

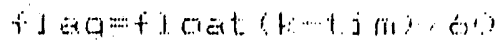

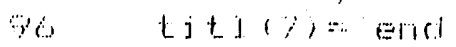

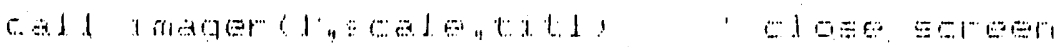

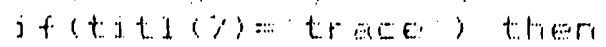

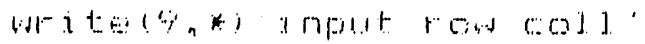

r.

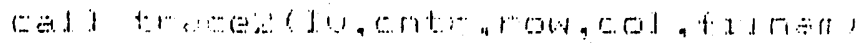

atoto\%?

erratis

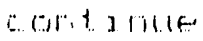

ing 
Source tides pucture.plo

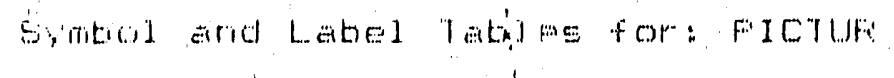

$\sin 11: 11$

$1:$

incl

r.cil al

(i) $0(1)$

- $\quad 10.01$

1

i

$1:$

c. 01

$1+\infty)$

$1[0[1]$

1. r.an

1

III

$1 i$

mots

L.) 1 : 1

b. 1 l:

(t.)

t.1. $1 \mathrm{~m}$

…. rit.

int.

int

ant

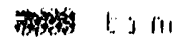

100

1 in

113

$\therefore 1=10$

101

1 Exith

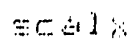

$=r . \pm 1 y$

$\therefore 1$

$t i$

$\therefore 1$

.11

$\therefore 1$

(3) 6.196

।

$\therefore$

MISTHE

(.) 11

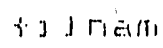

tis
T PF:

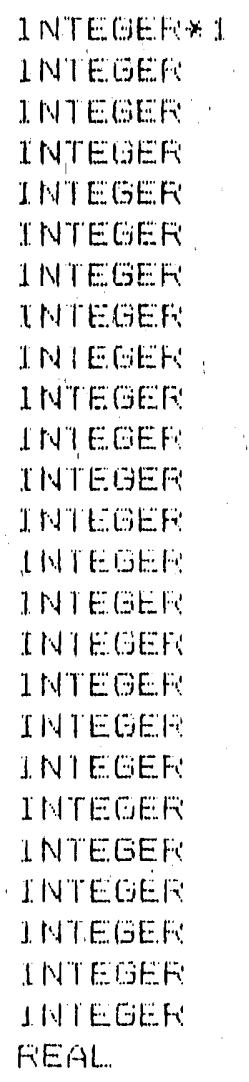

FEAL.

FEEAL.

rit: fill.

FiEfl.

H.: Fit...

FEFAL.

HIEAll

refint.

ril rill.

lition.

liter

ritionl.

refill...

IRE:AL.

lewill.

IEAL

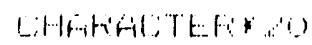

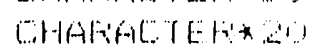

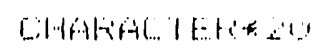

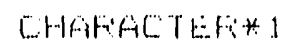

mllnte:6!s

Litutrituld

1.109 .10101900

0.16454101

014060

(1045654

(1) (1) 4 k.

(1) $(1)+56014$

$0(1456040$

00456644

(1) 4013060

(1) 445644

1015

"104560

(10)4ton.

$004665 \%$;

904561401

1)

$0404 \% 9$

$10456 \% 11$

0146014

(1) 456720

(10) 4 to 4

190468064

(101458

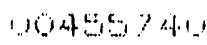

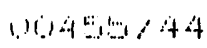

00010014,4

(10)(n)

01045090

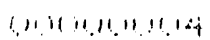

1.1146161019 .94

$110401 \ldots 1001$

61045, 45

(1) 1 (1)

mathes

.114.11.11.1

111.146461114

$11114: 361: 1$

1.11.1 616.1. 1

(11) 450101

111.14601

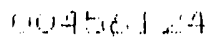

‥ 1406

$111611 \therefore .44$

11144519.101

, 11,1400644

111496010 bit.

1.) L. H. .

b:LLFI

1.1... J

$\mid i 1.1$
?.: I LAFICIE

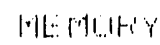
|. Ut,i-1)
15498
bit: I
1906 
bource File: Adoture.plo

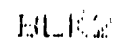
$3(1), 8$

L.Ailit:-1.

$\Delta ;$

83

its
(A1) DHit:s

(1) (1) $10(x)+0464$

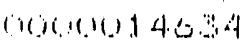
(10000) ; 1040
LAEEEL.

49

] 191.1

995
A Alublute 50

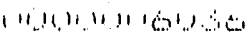
(1)14.12 () (1) (1).11) ] / that 


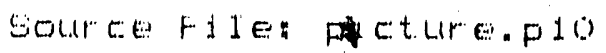

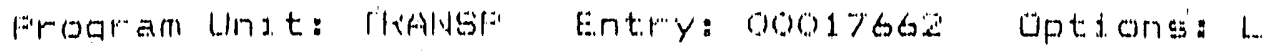

$000 \%$

9010

0004

00305

90.06

$0000 \%$

00.08

0009

$\cos 10$ *

$00.11 \%$

$00312 *$

- $00312 *$

$\cos 14$ *

from here on attach the subs for timang tre inter.

weations of a ray with a surface. note that

(1) $1015, *$

0016

0017

$\cos 18$

(ai) 14

(1) 120

orial

002

0023

00,24

0025

00326

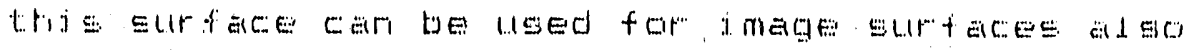

orser

0002

(1)

00

$\cos 20.1$

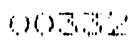

005

10.194

$(16)$

0030

0037

10306

1.1.) 19

10049

10034

10194

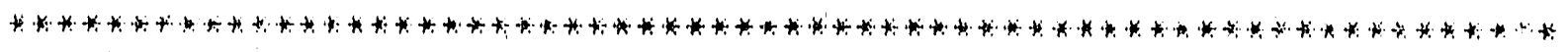

subroutine trangport (cntr)

1 mpd icit ricome

integer" cnt."

real in, rav (200, 23), t 1 ag, geo (50), 10$)$

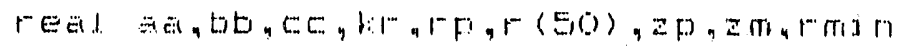

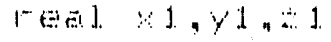

real ar, bo, chn, ap, bp, c.p, temp, tempd

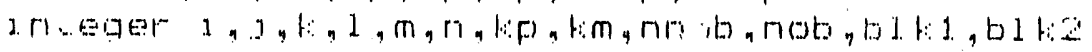

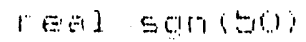

cher ater.

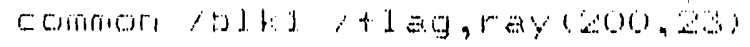

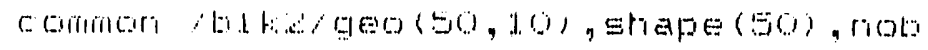

dat 21.5100

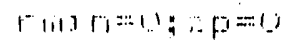

- 10.04

(i) 10.44

00.35

0040

10.54

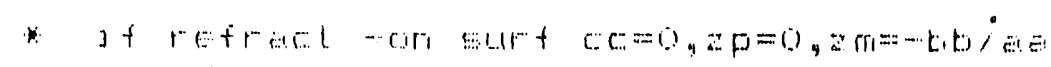

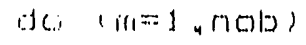

101,48

$0034 \%$

0050

0051

0035

Eelect casem (shape(m))

case ('sphere')

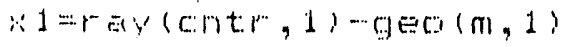

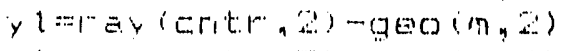

$z 1=r$ a) (cnt:r, -1$)-g e 0(\pi, 2)$

1.00354

0055

0016

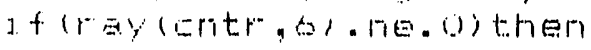




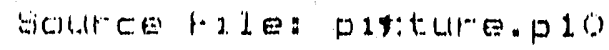

(1)

oo) 156

(1)

(1)

(1)

(1)

11006

(1) 104

(1)

00146

- 00507

0036

0.0305

$00 \% 0$

00371

00372

003

0047

bos?

1.10)

1019\%

00375

00379

00530

(NOEE

0038

0036

00184

0035

00180

$005 \%$

ores

वरा

OOE

(4)

$00 \% 5$

0046

00394

0030

0009

(1) $19 \%$

(10)196

10259

00400

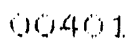

(10)46)

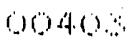

- 00404

00400

$0040 \%$

$0.040 \%$

00108

$040 \%$

(0) $41 \%$

(0) 411

(1). $41 \%$

$0041 \%$

0.1414 al $=1$ ir ay (critr, $\left.b_{1}\right)$ ir ay $\left(c, n t r, t_{1}\right)$

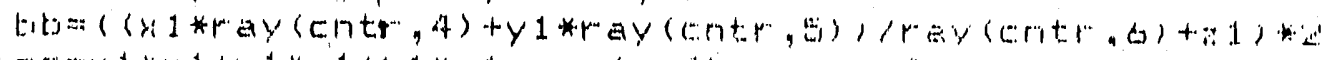

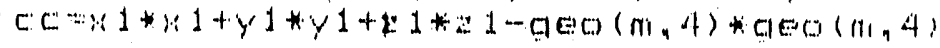

$5 c 111(m)=\pi 19)(1, a, E)$

$1+(0 \ldots .1+.0(0) 1) \quad \Xi g n(m)=-1$

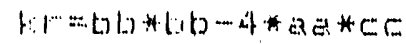

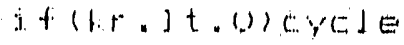

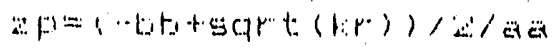

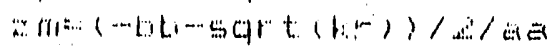

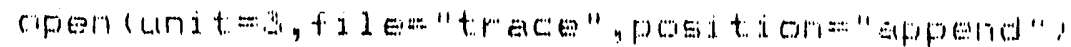

wr.

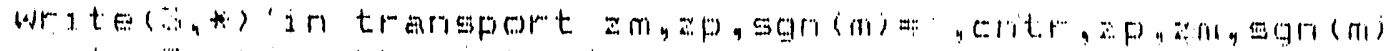

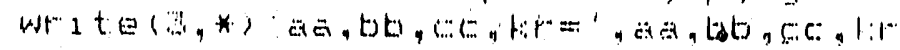

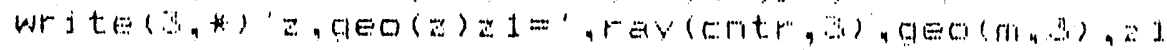

(ot $0 \mathrm{sec}(3)$

$1+(\operatorname{artg}(2 p)(001) 2 p=0$

$x+(a \operatorname{ass}(2 \pi)(10,1) a n=0$

* zр

in

$i+1 z F=0,1)$ then

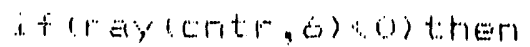

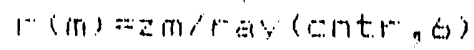

$r m i n=r \pi j] r+r(m)$

encidit

c. $y 010$

E[1

)

$3+(r a y+c n t r, g \cdot a)+r e n$

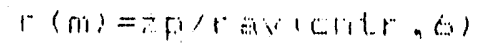

rind r r

crats:

c.i.is

incl 1

$3 \therefore-1,11,011$

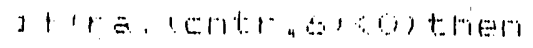

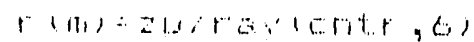

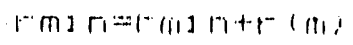

eriol

c) $>010$ ?

Eirlatit

$a+12 m$ min theri

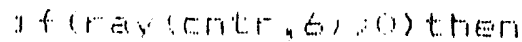

$1(\pi,=\pi m / 1$ a

$r m i n=r(m)+r m i n$

जाता

$\operatorname{coc} 2 \mathrm{c}$

खान

$$
\begin{aligned}
& \text { 1. } \left.+\because \pi \pi_{1}+1\right) \text { trenti }
\end{aligned}
$$

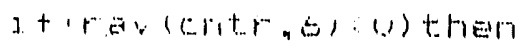

$$
\begin{aligned}
& r(m)=z m_{1} /{ }^{\prime} a r(c r i t r, b)
\end{aligned}
$$




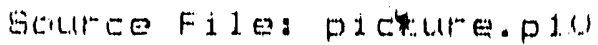

$0(141 \ldots$

0.416

$0041 \%$

()) 41.4

(1). $41 \%$

(.) 140

(1) $4 \% 1$

(1) $4.2 \%$

(10) $42:$

104.4

- (1) 420

(1) 426

(10) $4.2 \%$

- (1) 408

() 409

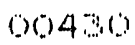

(1).4. 131

(1) 430

904.3

(1) 43 in

(1) 4010

(1) 446

(1) 497

(1) 4.18

10439

(10)4 41

(). 441

(1) 442

(): 443

00444

(3) 445

00446

(1) 447

(10)448

$\therefore 11449$

$01,4 \div 6$

00461

()) $45 \%$

(1) 445

(1) 145

()) 4415

(6) 4 the

(i) $490 \%$

- $\quad 11.14+26$ वान

0.10400

o[146]

- 0,046 (1) 49 .) 1044 0046 (1) 76 ociat? 00468 (1) 4 द 5 1.) 1010 $004 \% 1$ ()) 497

$$
r\left(\pi_{1}\right)=a p / r \text { aily }(c m, t r, \theta)
$$

exidet

eridi if

r. $n \pi) n=r(m)+r+m i n$

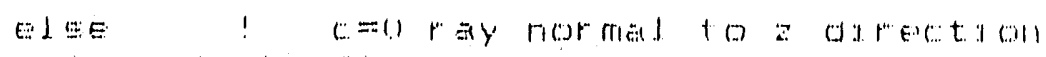

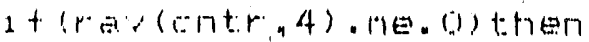

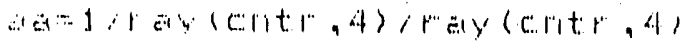

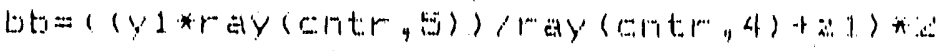

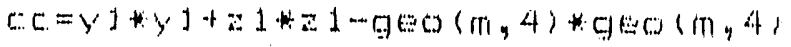

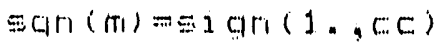

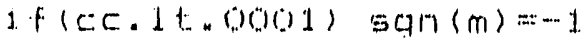

$t \therefore=t \omega * t, 0-4 *$ a

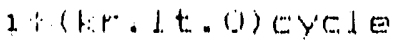

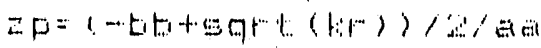

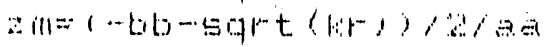

$i+(a t=(a p))+001) z p=0$

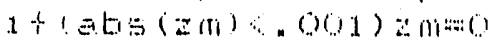

女 20 -

$1\left(2 F^{\prime}=i 1\right)+\cdots \in$

$j+(r+y(0) t r .4)(0)+b r a$

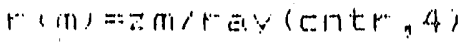

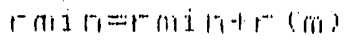

Endit

C...

alius +

$1+\left(\because m_{1}=(i)+r\right)$ trin

$1+(r a y(c) n t r, 4)$, (1) then

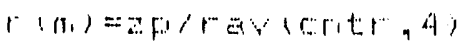

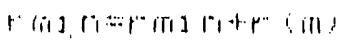

enat

C. $E \perp E$

aind

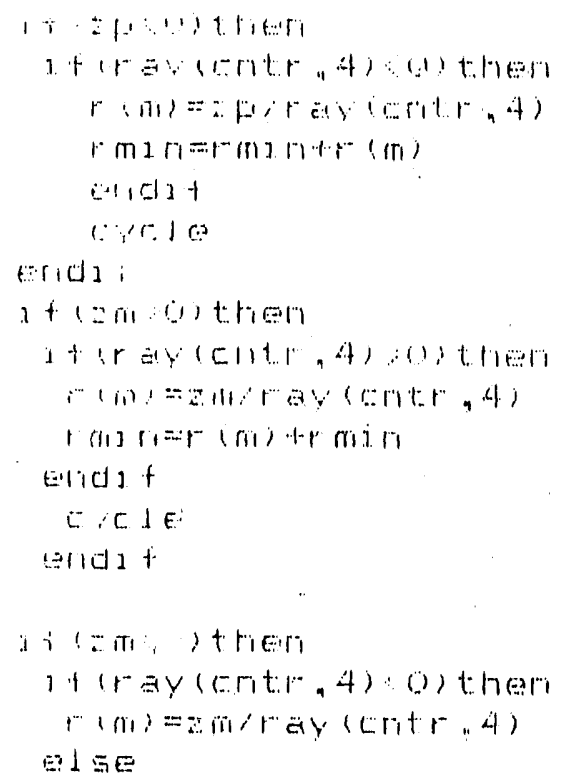




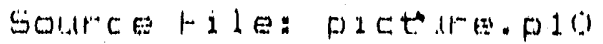

(1). 473

(1) 4,4

111.415

111,476

$\therefore 1197$

$111,4,16$

(1). $47 \%$

(11) 1401

(i) 1481

(i) $48 \%$

- (1) 4 E (0) 484 i.1. 48 !:

(1) 486

(i) $48 \%$

(i) 4 Hध

1) $481 \%$

(1). 490

(10. 491

(10.) 492

(11) 49

(1) 494

(.) 495

(0) 496

() $49 \%$

(1) 498

01049

(.115.11)?

(1)

(1). $1.1 \%$

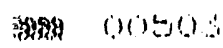

(1) $1(2) 5(1) 4$

(i)

(1).5060

(1).

(1)

(1)

(1) 510

(1) 151

$\because 110.1$.

(1) 141,

(1i)is 1

(1) 515

(1) 19116

(i) 11 ?

(11) 11.14

()i) 19

- (1) 10

(1) (1)

$(11,1502$

(1) (1) 1,25

(x) 1024

(1)

(1) 520

- $11062 \%$

00.68

(1) 61,19

(1) (1)

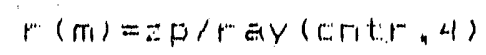

eincil 1

oriclat

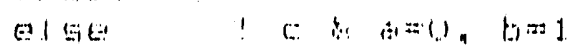

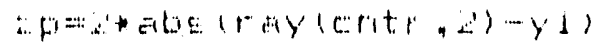

$1+(2, p+1)$ a

$1(t)=\vec{r})=0$

(a) $11.21+$

eridjt

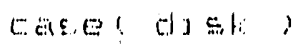

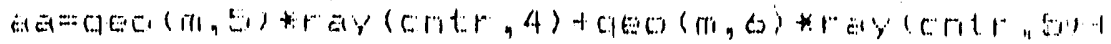

$+$

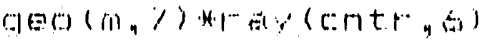

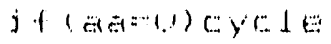

$\therefore$ r

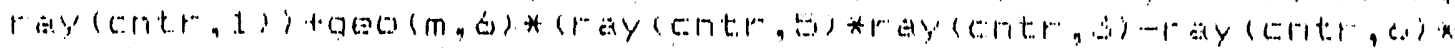

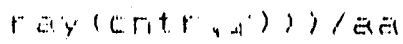

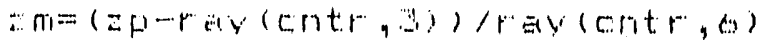

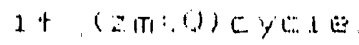

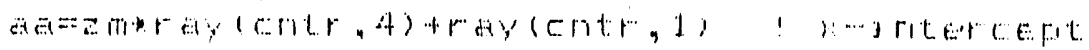

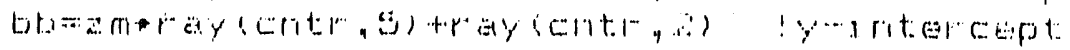

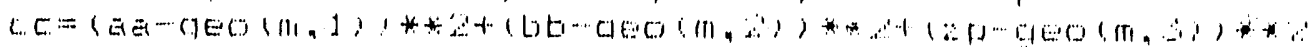
$-(100)(11,4) * * 2$

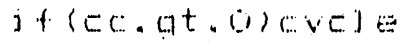

$r($ iा $)= \pm \pi$

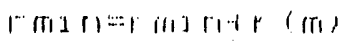

C. $650+\therefore-100.1$,

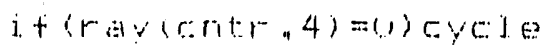

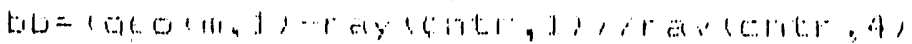

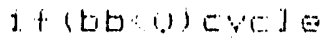

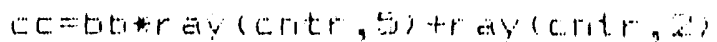

$1+c c)$

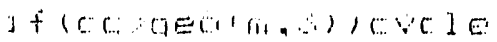

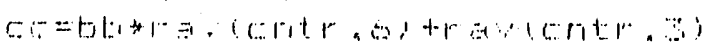

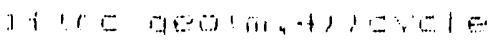

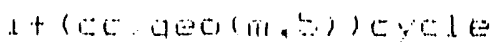

$r(m !=1, t)$

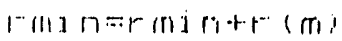

$6-15$ e $1,-1,0.1$

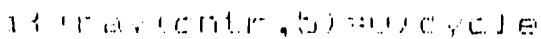

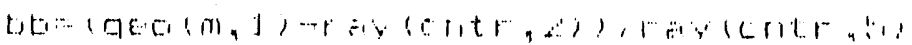

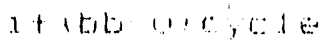

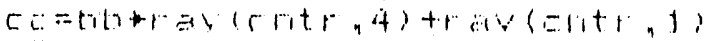

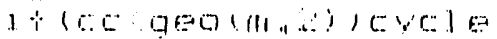

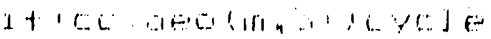

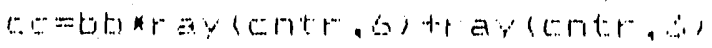

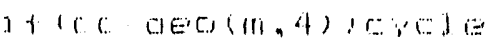

$1+100$ o

$r(\mathrm{nl})=\mathrm{mb}$

$r \cdot m i n+m a r+r(n)$

C.

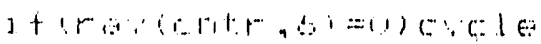

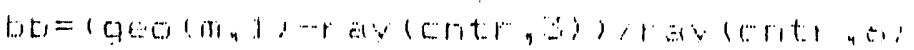

$1+(b 0,1) E y c]$

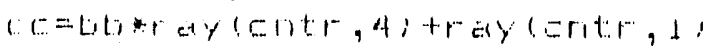




\section{Sulle lides pacturedpdo}

(1) 14,1

or.

(ac)

(1) 10.14

(1) 105050

(1) onster

$005 \%$

60548

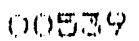

106401

- $\log 41$

(1) 1542

(10) 143

(.) 10544

- 00545

010040

$0054 \%$

0.15489

10.1544

00651

ouses!

()

colos

00544

0065

00585

$0056 ;$

0,058

00659

bosec?

0.01

(1) 502

01063

0064

1.06oly

1.10156

(10)?

$\cos 568$

orits

(1) 1) ?

(1) a $19: 1$

(1) $1,1,8$

(1)

$1016 \% 4$

" ocos

1)6

(11, sisi,

- labe

(ii)

(1)

01501

ogtiets

arses:

1). 184

19)

(1)

(1) 1096

ariget

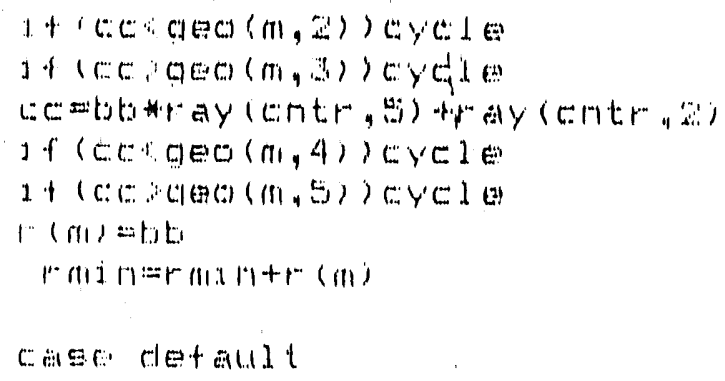




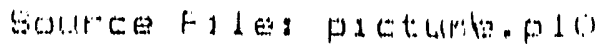

(1).

(1) 1090

(1) $10,5,1,1$

(1) $105 \%$

(11) 5,5

(.1).

$1,110,145$

1.11.50 50

11065 ?

011546

- 1.11 .594

(1) (1)

(1) 10001

(1) 60 (1)

(1). ह) 60.5

(1). 62104

1). (1) (1)

010606

$00 \%$

مा.

(1). 6010

0010

(1) 611

ois 1

\%(1)

()) 1 1A

0016.15

orotc

(1)061\%

(1) 14

(1) $10.1 \%$

(1).

(1) 61

110

(1)

1100.4

(1) 620

(1)

1062\%

$100 \mathrm{cos}:$

$1106 y^{1}$

(1) 1104

$101 \%$. [1

काष

- 0069

(i)

(1) $11,1,1,4$

- (10)ento

(1)

c)

01060

006401

(i) 1,41

(1) 62

0064

(i) 64.1

$(1,16464$

1.1064

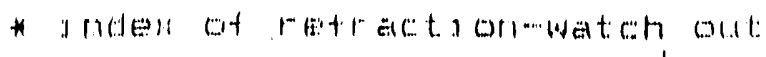

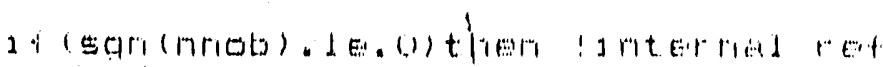

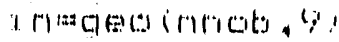

(t) 5

eridit

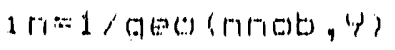

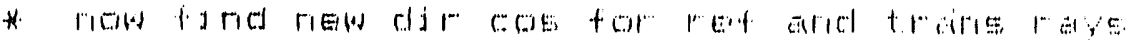

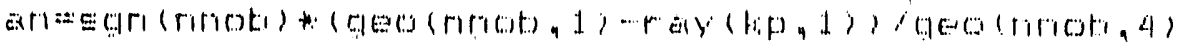

br $=5$ a

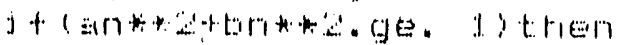

(a)

$1 \mathrm{~s} \in$

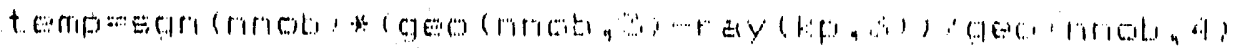

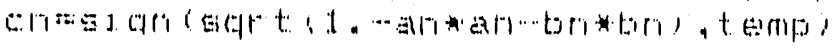

Evide

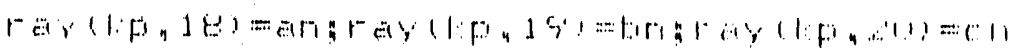

(1) a) (f,

$1:=0$.

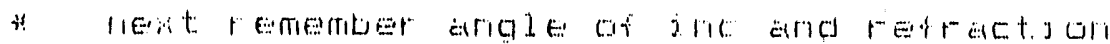

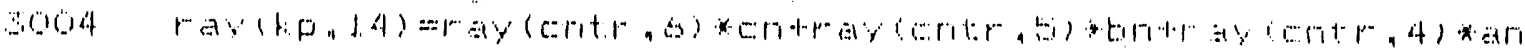

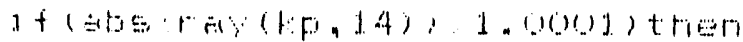

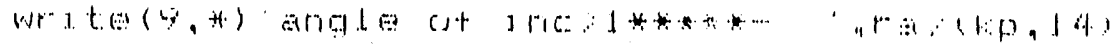
EMdit

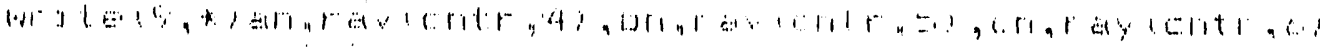

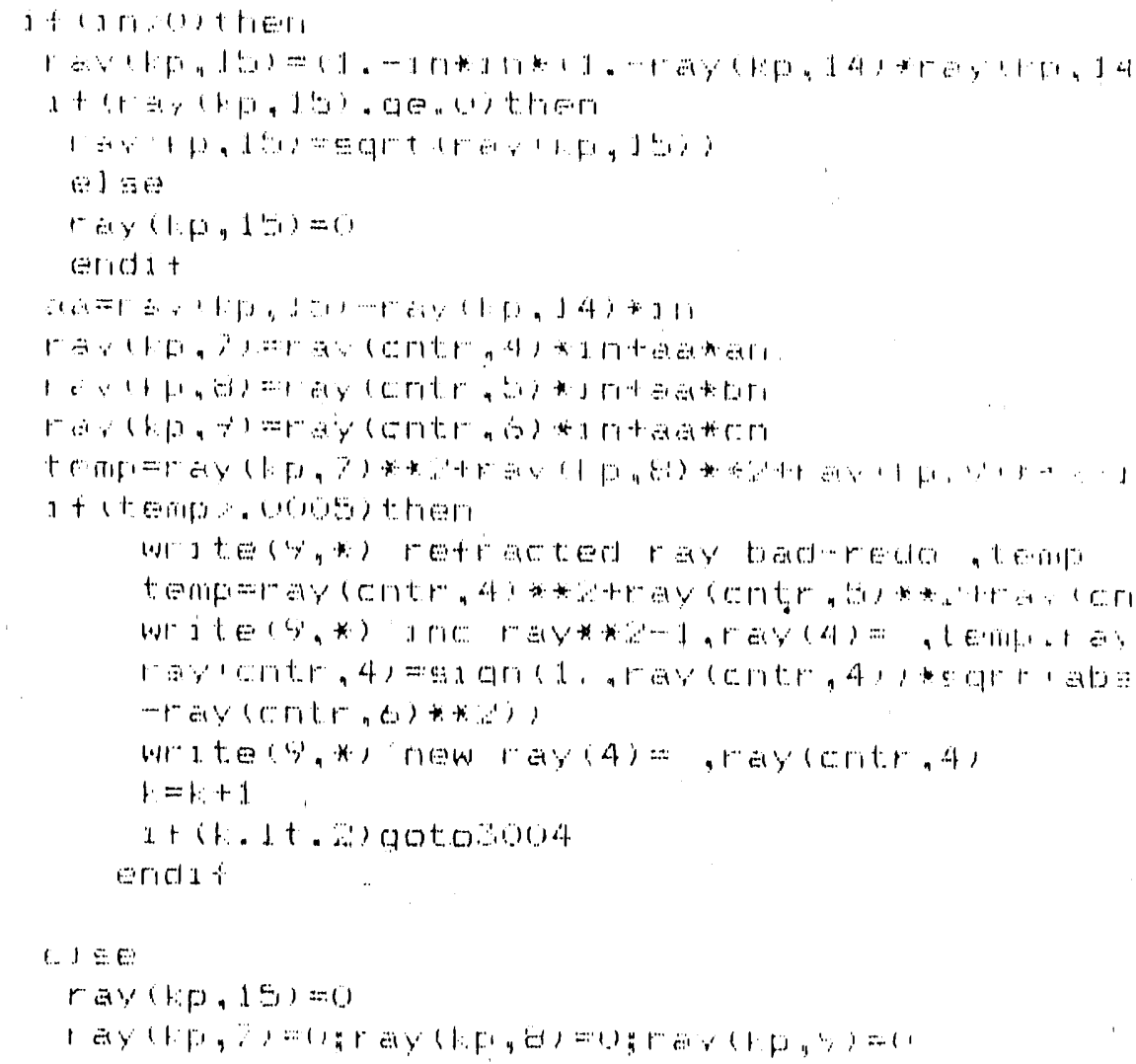




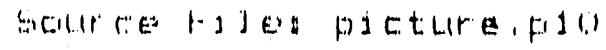

(1)i) $44 \%$

(.). 1., 46

(1) 649

(1) (1)

(1)651

(1) (1)

(1) 1066

(1) 1054

0005

onstic

0004

00648

0064

00196

- ocotol

$0006 \%$

0006

00604

Endat

10106:

(1) 1060

Dinter

oobes

$000 \mathrm{~s}$

$00 \%$

006\% 1

$006 \%$

(1) $107 \%$

(1) $16 \%$

(1) 167 .

010676

at 00077

(W)

bowe

(1)

(1) test

6060

(1) abes

a)

$006 \theta^{2}$

111608

DORE.

M)

(x)

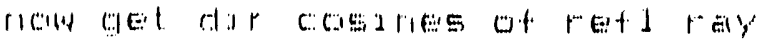

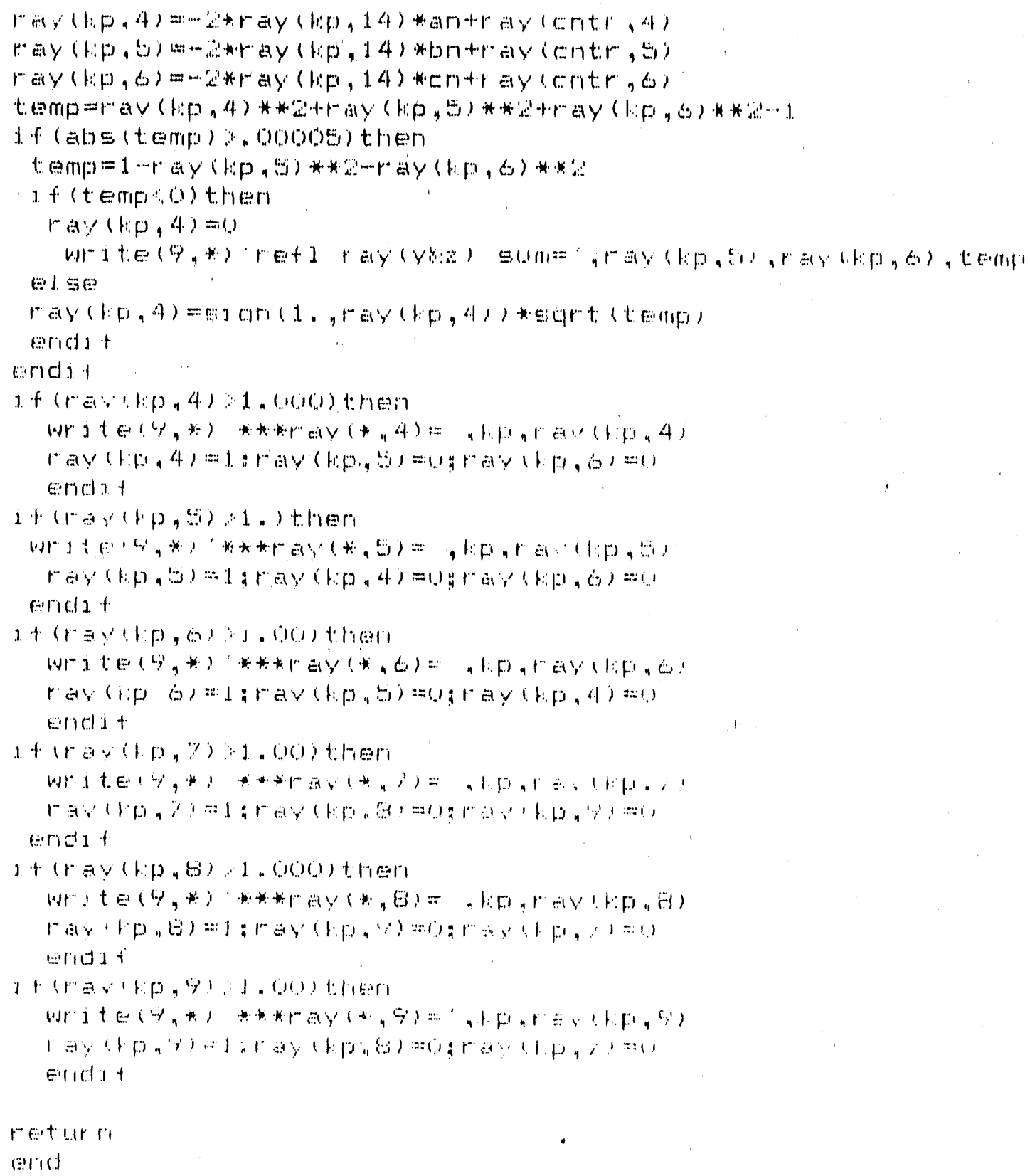




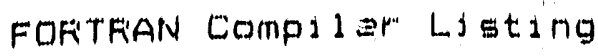

6)

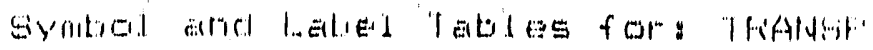

S MULILI.

c.ritir

$1 \mathrm{rl}$

rav

+1 a

- ger.

an

bu

cc:

$\mathrm{lir}$

pros

$r$

$\Sigma p$

EIII

rnis

$x 1$

y'

z. 1

ari

bri

cil

Exp

bp

cP

t.

sin temind

1

I

1

I

"11

11

$1: p$

tail

ririol

rict:

bil 1

b) $1 \ldots$

5.7.711

* Elater
ソYFE

INIEGEH

|AE:Al..

FE: FIL

Fit:A Al.

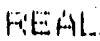

FiEAL.

FEEAl...

Fitrifl.

Fitefl.

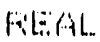

Fitest.

HEFIl.

rite

riteril.

rit: fill...

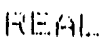

ritefl.

Fl:fil.

ritent.

lif::Al.

fitefl.

FiE:Gl.

价fl.

fitzinl.

fit.ibl.

IMIEER:

IN EGات

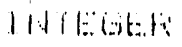

111 E G

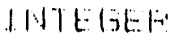

INT E L F F

[N] EGER

IINIFloteri

JITEERE

I d I E G t t

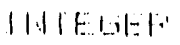

IN E LE:

l.t.tel.

c. Hethe If:
ADDith

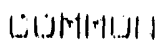

1)LII:191;

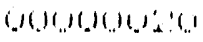

c)

$(10,10)(21) 10) 14$

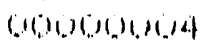

(1)(0)(5)! 4

0600001010

ocos)

$(20) 000 \% 40)$

$(1021010) 1044$

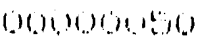

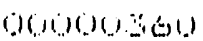

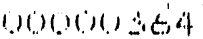

U11011),

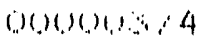

0000040.10

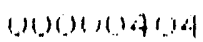

(0)11104410

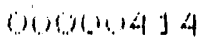

110001420

(1010)(10.14.

600.10 .48

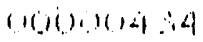

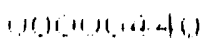

(1)11111444 at

(16).11).

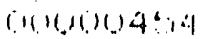

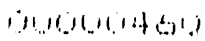

1.111111.12tat

$1019.1011+4: 11$

$111,1010110+4$ a

1010.106.

(10)101, 2.)

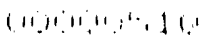

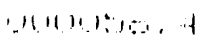

1101010514

$111119.11 .8+29$

11011010.94

111114.8 4

tith $1: 1$

BL. H: 1

IIL I I
$1: 11 \ldots$

t:il $1 .:$

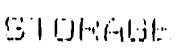

1. Lill.pil

bil. H.

til. 1

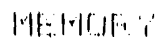

!... it?

andies

18406

LAFIE:L.

HDPH 65

L.ABIEL.

H1.101. 45 
Sotorsta

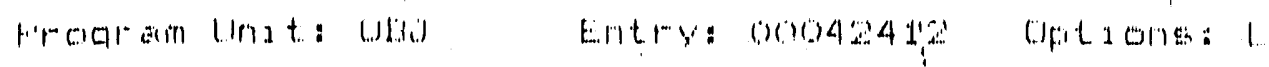

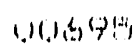

(1) (1)

(1) (1) (1)

(1).

(1) $140 \%$

$10 \%, 101)$

(1) (1) 101

(0)

$0.1 \%)$

00764

(10)

- 0070 os

(1) 190

(10)

610\%)

C.

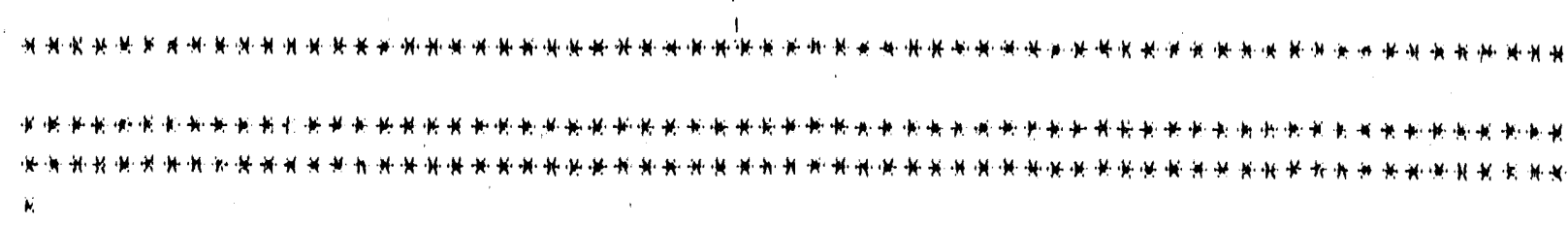

(i) 10

0011

$0 \% 14$

(1) 1919

(.) 140

(). 19

(1) 16

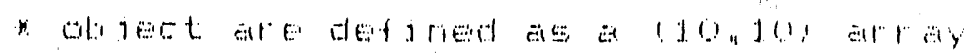

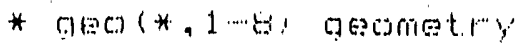

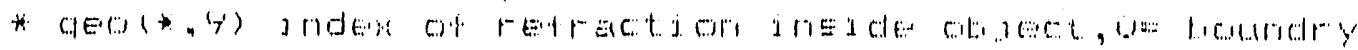

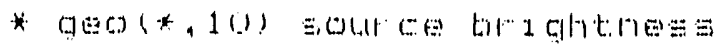

(1) $\%$

$00 \% 18$

(6i); $; 19$

कo,

(i) 1

(1) 1012

(1) 10.4

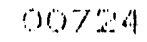

(11, 11,0

$11,1,16$

(1) $117 \%$

(6) 1970

(i) 729

0070

(1) 197

$110 \%$

(1) 110

(1) 14

(1) 19,

- 19878

(1),

111,98

(111\% $\%$

119.

(1), $; 41$

$110 \%$

(1) 14

(1) 144

(1), 4

(1), $: 48$

111.4 ?

(1). 48

111. 49

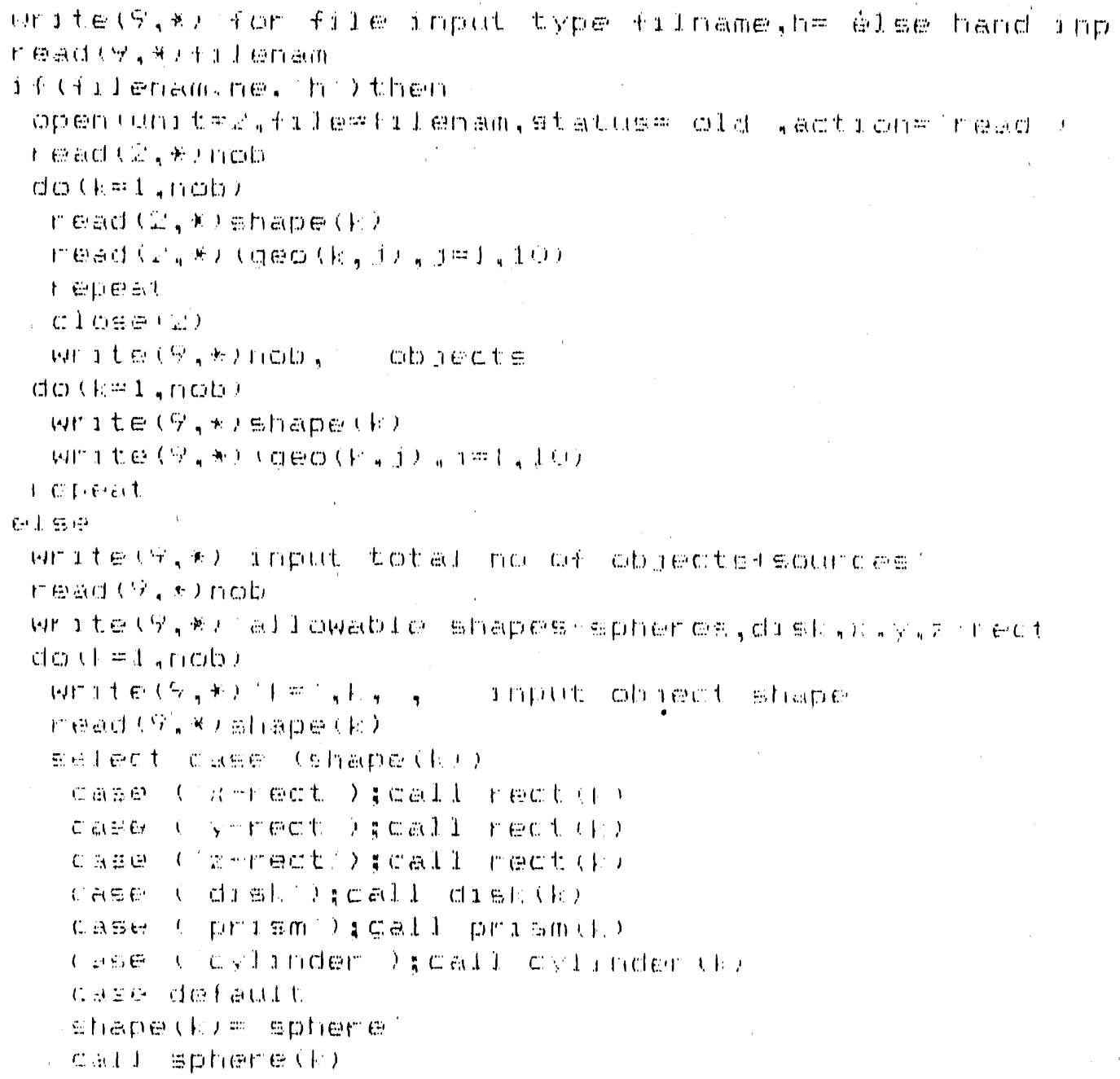


औ11) (1) 11750.1 (1) 192 (.11) 110 (1) 14 (110750 (1). 75

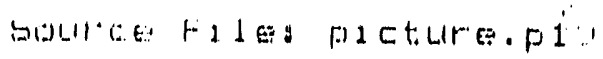

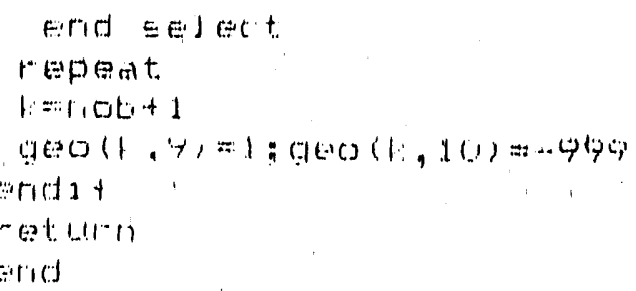


source Faden pleture.pla

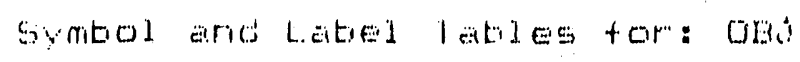

5YMlitil.

t:

q

rioto

j

- 1

in

n

bl:

- griepe

ti j. eriam

\section{STOFAEE}

1.. OCFFl..

I:At...

\section{TYFE}

INTECEEF

FEEA

INTECHE

INTEGEF?

INTEGFE:

INTECEF'

INTEOEFI

JNTEGE:

CHAFALEFE

CHATAACTEF
ADDOEE

(1) $(11+119$

60101014

(x)

orocolosen

(6) (6)

ocoonowen

ocenored

010)(16)

()

(6) $161 \%$
GuNATH

LiL...t:

bLI...t.

$131 \ldots$
ME:MTIJU'

60

Wo

I..AEEI

ADIDFESS

L.FLELL.

AUMtEEG， : 
Sultre F̈i le: pleture.plo

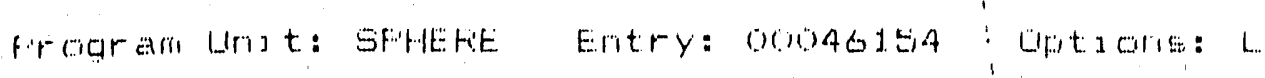

$0075 \%$

00758

010759

00760

00761

00762

(). 0767

- 00764

0076

00760

\%)

$0 \% 06$

00769

00770

00\%

$007 \%$

(w) 13

(1) $17 \% 4$

(10\%)

$00 \%$

oor 7

() 1778

00799

$007810^{\circ}$

() 7 है 1

o)

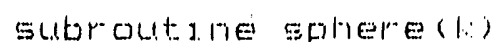

3. Mipl jejt mone

red

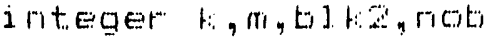

char acter*20 shape

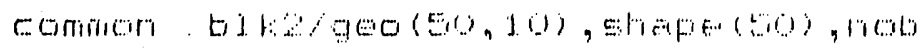

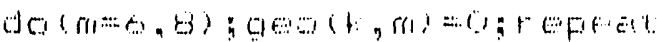

4.1.3. te (

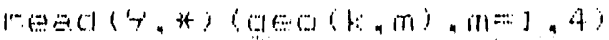

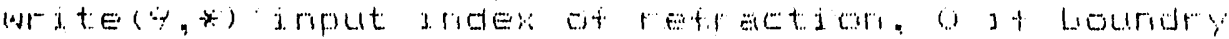

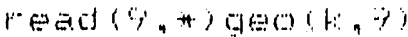

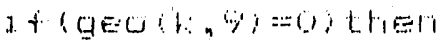

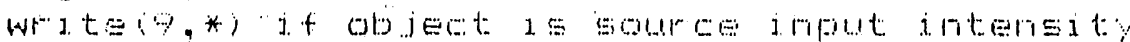

l. Eed $(\%, *)$ G

๑) $\Xi$

Ge: $0(1:, 10)=\ldots 1$

endit

$r$ Etitir

काल 
Source File: pisture "Fior

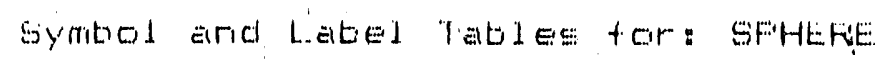

GMUELL

$1:$

aed

ai

b. 1 t:

- riob

stiapez

STOFALE:

L. UCOABL

ELL

L.ABEEL
TYFE

INTEEEF

fitifif.

J. NIE EEFI

INTEGEF:

1. NTE GEFF

C.HAFACTEH
ADDFFES:

$[\mu 11+11 \times$

01000004 001010000 0000004

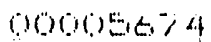
00103724
Cold

El.

BLL.. F. St:

E1...
mHertoris

24

$\because 0(0) 6$

ADDFESG

L.tibiteL.

AUDFES 
Soldrce File: p'seture.plo

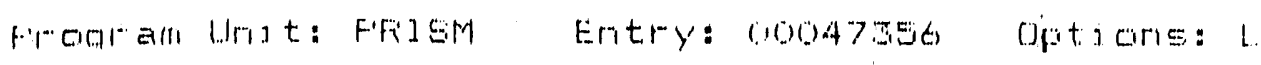

01084

(1) 796

(1) 786

(1) 197

(.) 1988

() $78 \%$

(1) 1990

- (1) 791

(1) 179

0074

(2) $7 \% 4$

00795

$00 \% 50$

(c) $19 \%$

(10) 798

(10) 790

()080\%

()

10802

$0080 \%$

()) 6004

00005

()

(10)60\%

0000)

\%)

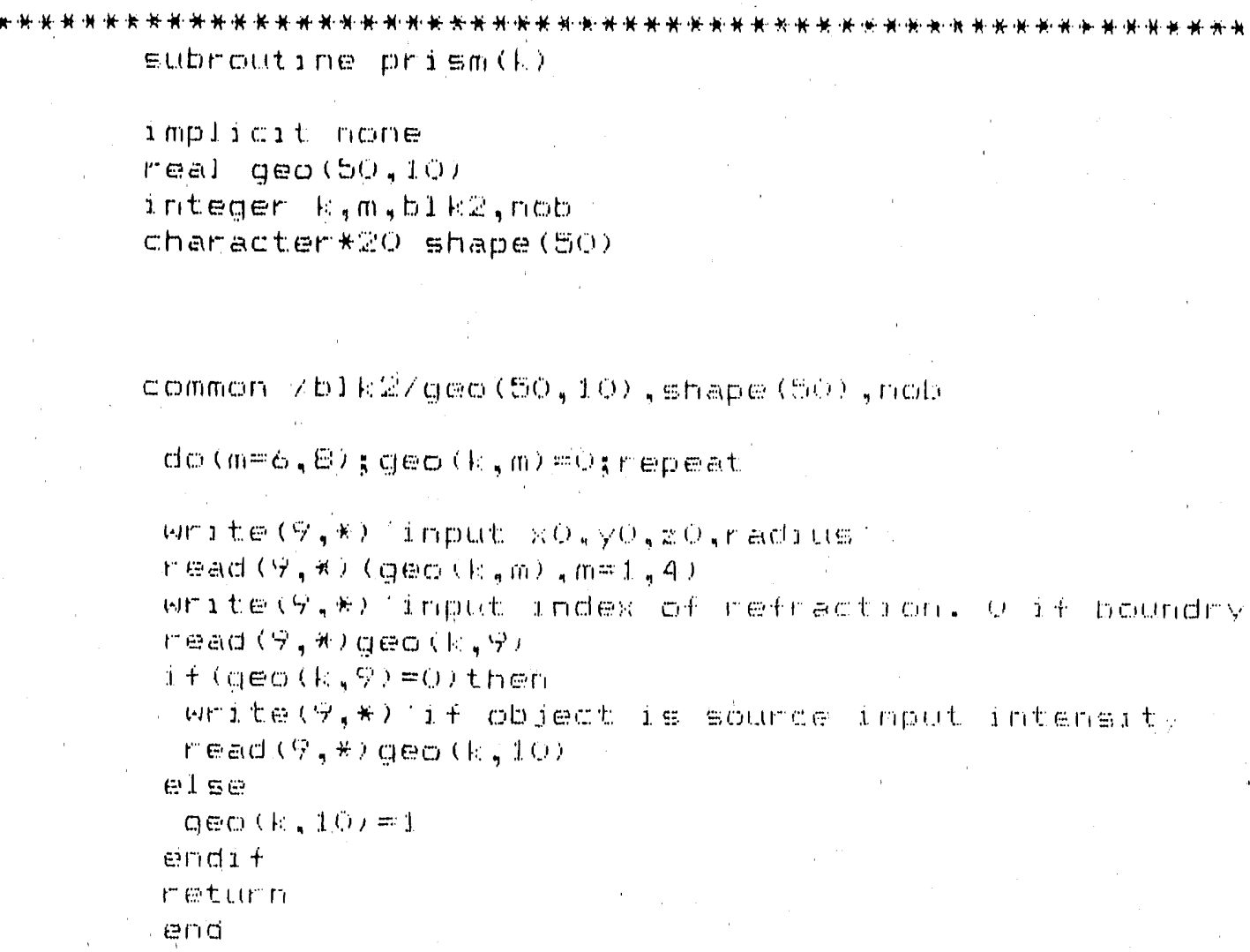




\section{soluce rislen picture.plo}

Symtod ardo hated latedes for: Fridsm

SYMEIUL

$1:$

$\square \leqslant 0$

iil

b) 1 ti:

nots

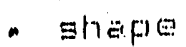

$\left|Y^{\prime}\right|^{\prime} k:$

1WTEGEF"

FEAL

WWEGEF:

INTEC:E

INTEEEF!

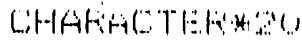

HLilatiog

buा

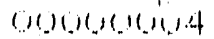

(1) $1010(10) 0$

(1) (1) (1) (1)

(1) $10(0), 24$

and 10104

MEEMINYY

G) LIFAGE:

L.CLAHL

IiL 1

$$
24
$$

solot

L_AEE:.

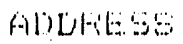

L. Filut:

आ以山1 50 


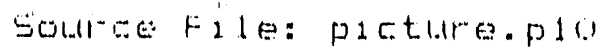

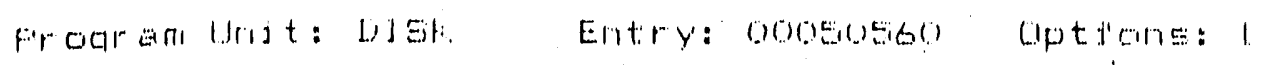

1001010 (1) (1) 1 (.)181: (1) $81 \%$ ()) 814 0015 0010

- 01017 \%) 18 (). 819

(.) 820 0021 01622 (i) 8 . (1) 124 (11) () 100.6 (1) 60 " ? () ()) 0) (1) 61 (1) 100 006 ()) 834 (c) (1) $814 \mathrm{c}$

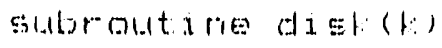

1 mplacit norien

racal cate $(50,10)$

integer $i, j, b, m, b 1$ b 2, not

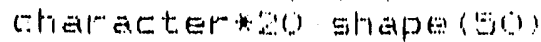

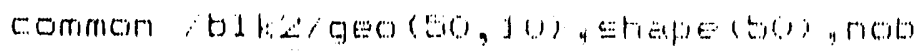

$d 0(m=6, \theta): 000(1:, m)=0) ; r \Leftrightarrow \in t$

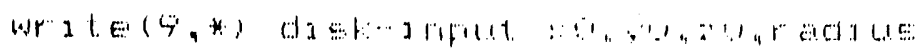
r

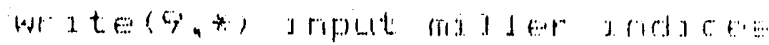

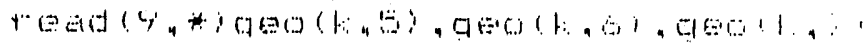

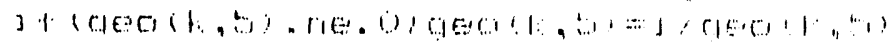

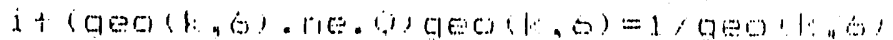

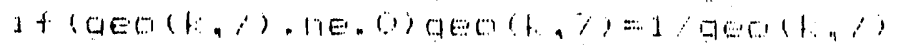
(a) $0(1, \%)=01$

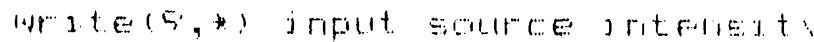

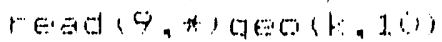

retirn

Enrd 


\section{Sontres Fid le: ploturenplo}

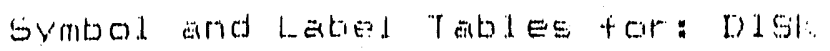

SYM1:01.

1

$a+0$

1 .

if

- iri

b) 1 r: 2

not

sticise
$T \cdot F$

INTEBE:

ritint.

IN" EEEF

INTEGEI

] N'I EEEF:

INTEOEF:

IN EEGl: tr

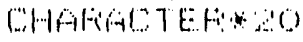

Aldititision

burlmy

(10)1010.114.

()olom

o(o)

or)

(1) $10(0)(1)(1)$

0000 bo 14

o(ocomat
I.) (17)

111..1:..:

I:il.. 1:

[:11, 10:

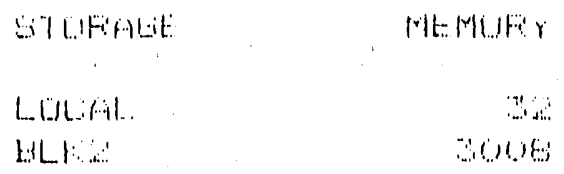

L.ALEE.:.

Ablates:
L.. HIELL

molotess 


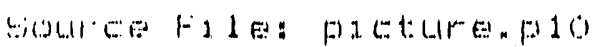

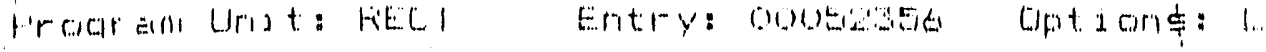

1013411

(i) $6: 41$

$\cos 142$

1.101943

(.). 844

00145

0) $34 t$

- $\cos 44$

00848

(0) 149

00850

o.

(1) 85

085

0064

OQBE

(1) 106

(108:-

010808

10854

$\arg 800$

(a)

noistis

ooges

Gitor out: 1 re rect (b)

inpid ja. at none:

rest aeo $(50,10)$

1 riteger k, m, bit:2, not

thar acter-*at shape (s)

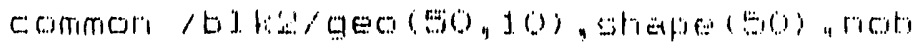

Gen (1, , i) $=0$ a $\quad$ geo $(1 ;, 7)=0$

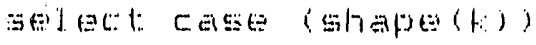

ti.

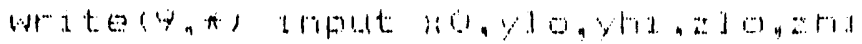

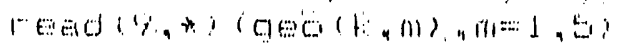

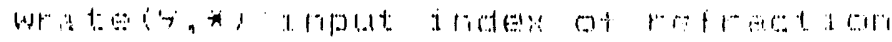

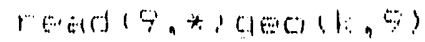

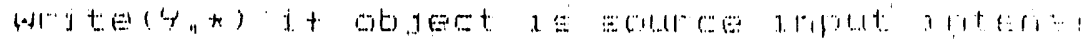

$10 \cos (\%, *), 000(1,10)$

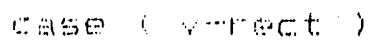

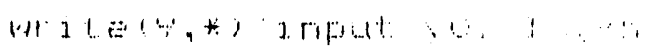

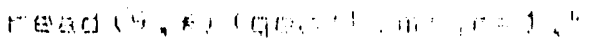

1.4: i

1,101004

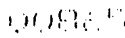

: 


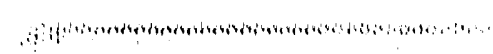

| IIII

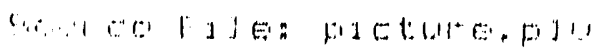

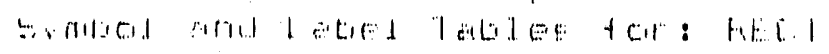

\section{$6 \times|4|: 1)$}

1

nenton

$\pi$

b] $1: 2$

vicity str $=00$ :
Trlet:

1 U1 E II.

|tint

J lill. blet:

INTEGL:

J I I FE: LEt:

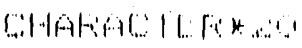

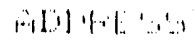

$\mid: 1111\} ;$

1.11011.10.110.19

$11+11+1,1, \ldots$

$0119411111, \ldots 1$

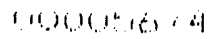

(1)11010?
1. 1.11111011

1i1. 1:. :

1.91 .1

$1: 1.1$
L. inlitist.

ml. $1.1+2: \cdots$
1.1F MIIIFy

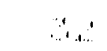

$\because 11161$

$1+1.1+1.1 \cdots$ 


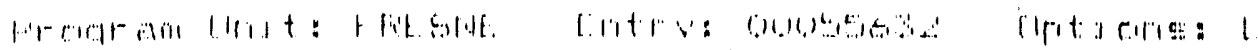

$1.16197 \%$ 111)

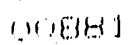
iा) $110 \mathrm{~B} \%$ (1) 1884

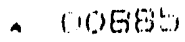
(1) 840 (), EEs? (1) 863 (1) $856 \%$ ()०8\% (i) $8 \% 1$ 111.18) 00959 (1) 8994 (i1) i)ers (1) $10 \%$ (1) 158 11189 1119.90119 $8.10701]$ 1090 1) 109414 00965 $1+4 \times$ लिखा (1) (1)

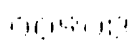
$(11, \cdots, 11$. 1119. 11.

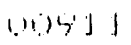
(10) $1:$ 11013

$(11 \%)+1$ 1111. 1 $1: 1,16$

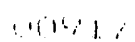
1961 ind ]

- 1118. 111 $\cdots, 1$

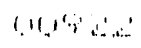
$111,19,25$ 010924 11981 11196 (11) 10,4 ब1, ब. $6145 \%$ 4.11' 119.41 $110, \therefore$ $111, \because \div$

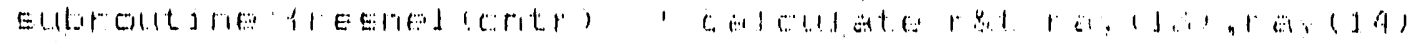

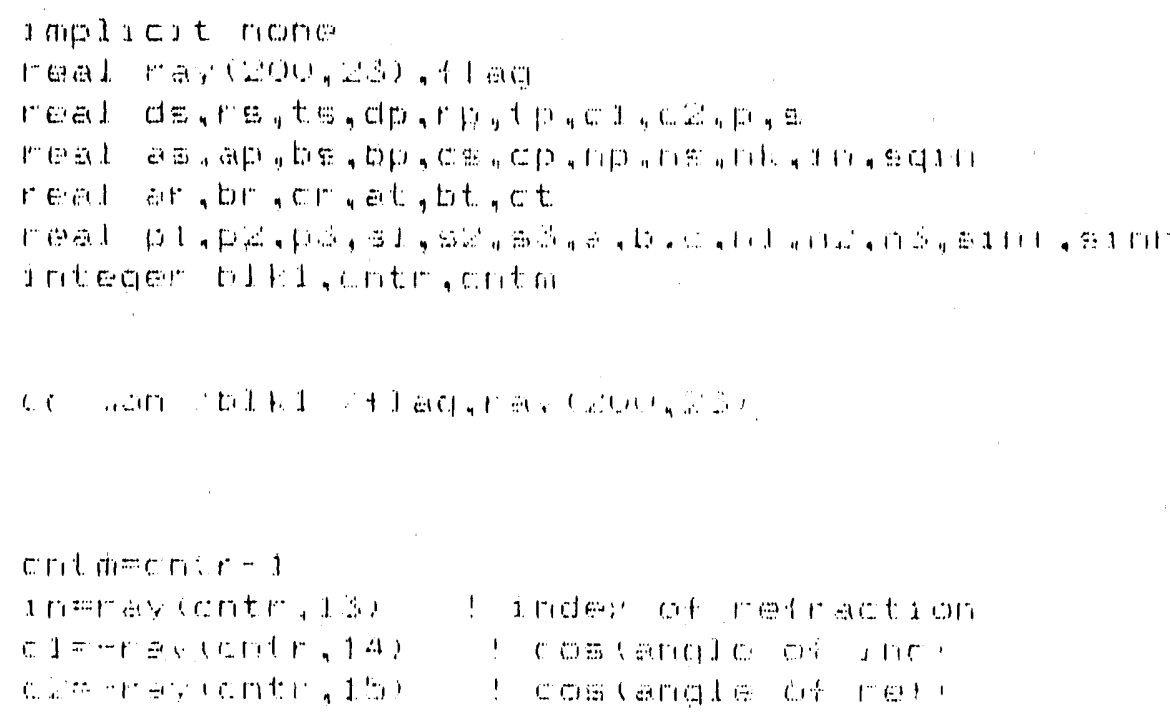

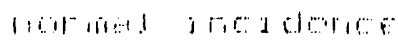

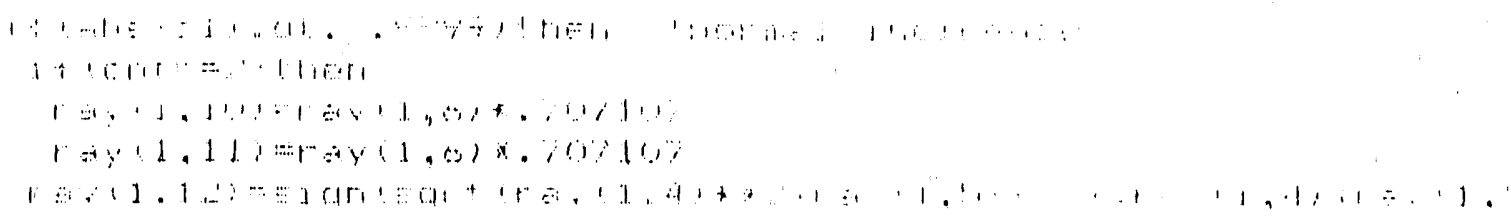




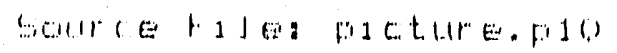

1.1914 .94

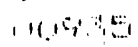
(1) 110.26 (1) $119.19 \%$ 1048

\section{$+\pi * *$}

$1019,01 \%$

(i. 190940

- io.

(i) 942 (1). 194 (1) 194

- 101045 ता) 446 ?46\% 1116) 1119 19 1119641 1 140101 111960 41958 . $10 \% 4$

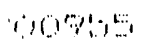
$149 \mathrm{cos}$ $\therefore 1,5,5 \%$ 164 1.21959 (19) 619 1996. 10196

$\therefore 119803$ .19t 1. 461

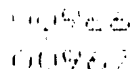
(1)'60 ares 1 1 1 11$$
\text { 11 } 11
$$

a $1, \cdots$ $.1,19$ 1. 1...' 1 - 1.. 1

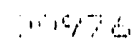

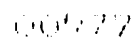

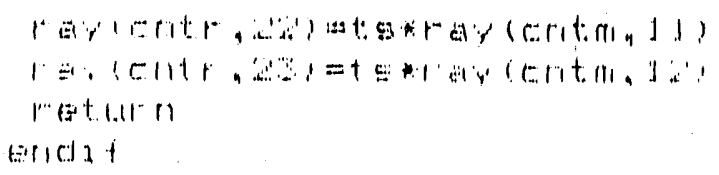


$1111 \% 1 \%$

$1,1,14,6011$

1110,4011

110968

111,9696

- 11, 1018 10101,964 1) ) (1) हैe

- $19.198 \mathrm{~d}$ ?

11) 4 \&

(i)(

111,1419

$1.11,4+2.1$

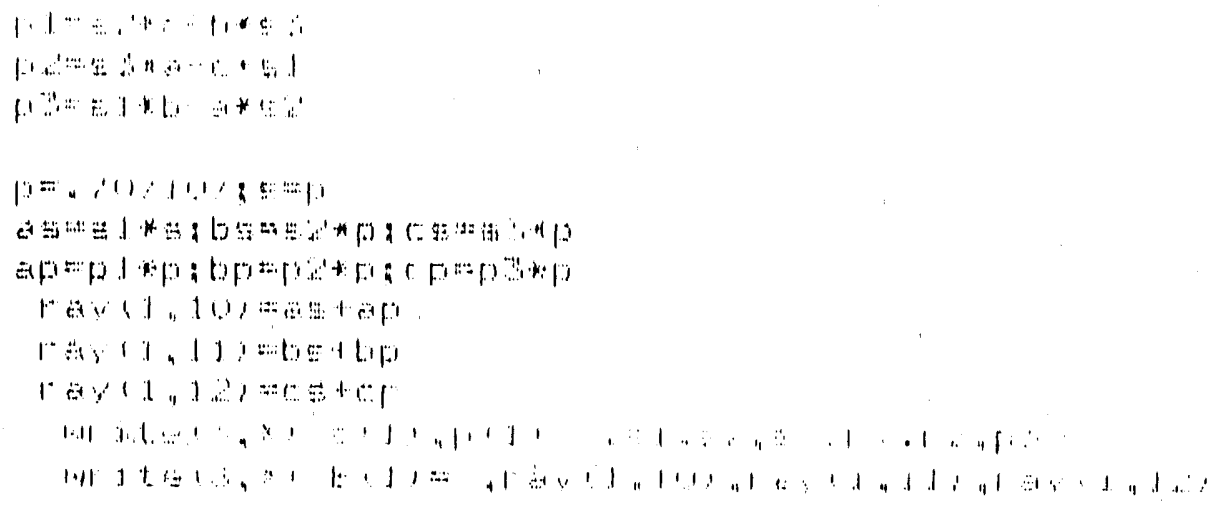




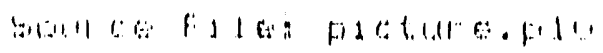

$111.91118: 3$

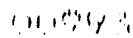

$111,1,1,1,1,4)$

E. 1.1.

$118,1,4,4,9$

$1,11,16,16+1,19$

i $11,11,19$;

1.11 .169763

$1.647^{4}$

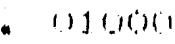

(1.1611.1.

(.116)

a) $1610 \%$

111014

$111+1 \cdots$

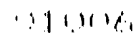

$\therefore 110 \%$

0111.19

$111,11.1$

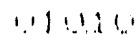

11911

114.1.

1) 1.1 .19

- 11.114

1) 1i)

11010

$\therefore 111:$

.11119

(1) 11) $\mathrm{c}$

$\therefore 11 . \therefore$

111.21

.1192

1 J is

$\because 1+1,4$

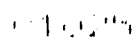

11.20

1) $11.12 \%$

11] int

$111,1, \cdots$

ग) :

i $11 \div 1$

$11, \because{ }^{\circ}$.

11. .

$11119 t$

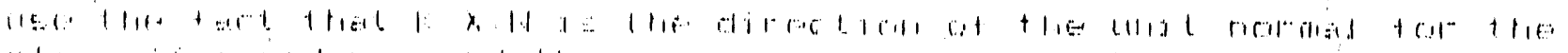
bl

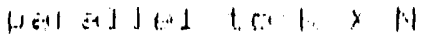

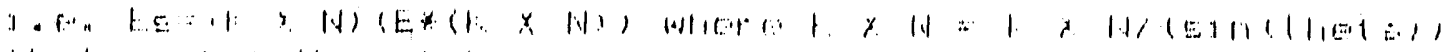

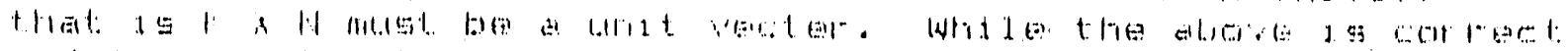

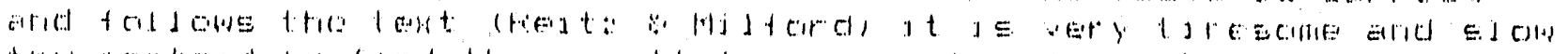

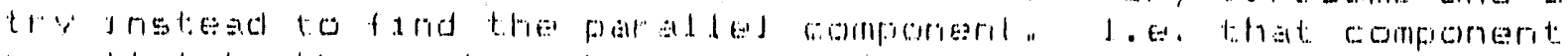

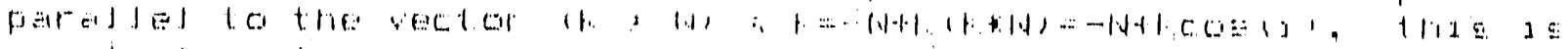

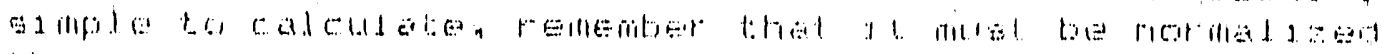

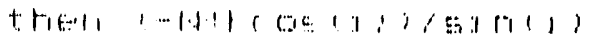

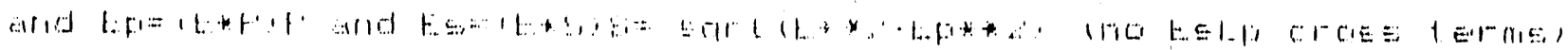

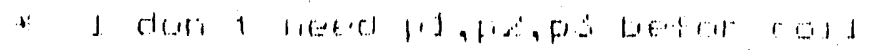

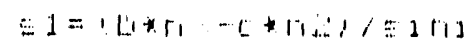

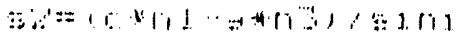

(6)

"

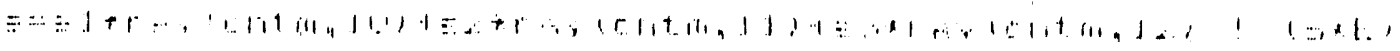

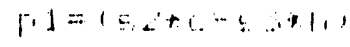

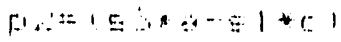

$11 \therefore=(\mathrm{c} j \mathrm{j}+1, \ldots+\ldots)$

$1 \quad \because \quad 5 \quad x \quad 1$

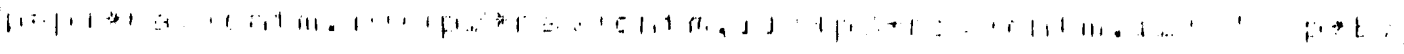

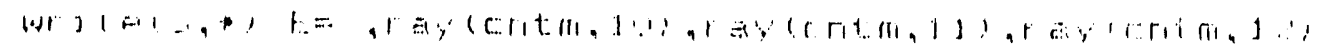

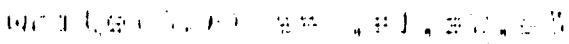

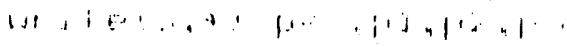

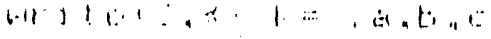

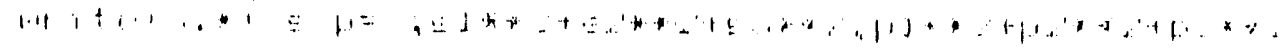

$(-1,0) 1$

11..."

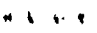

$+11, \cdot$

11,1

$111, \ldots$

$1,1, \cdots$

11111411

111.44

111.4 .1

- $+1,1.1$

111,164

$1 \because-4 ! 1$

$\because 21+1,1,1$

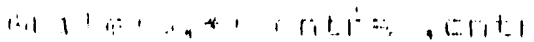

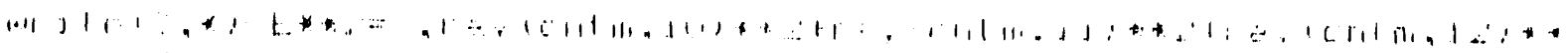

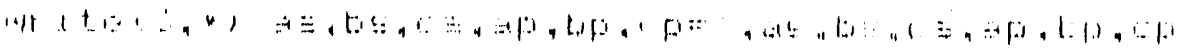

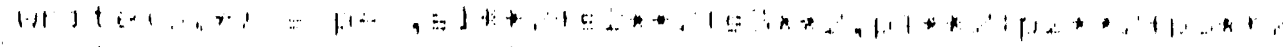

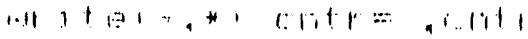

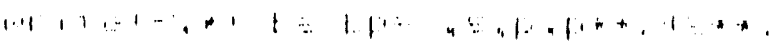

an 
1.11446 () $104 \%$ 1.1.1.1483

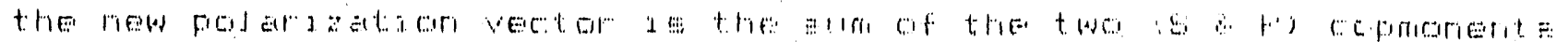

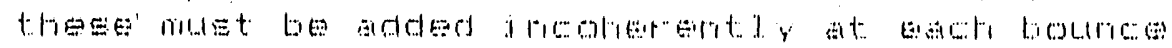

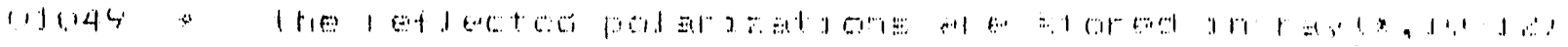




\section{Goureeflde: Ficture.filo}

111164

प11

․ 1110

i1111

111 19

$\therefore 1 \mid 1 \therefore$

$\because 1114$

$\because 111 \%$

1) 116

$1111 \%$

- a11 16 1) j. $1 \%$

i112.

- ग5 1

$\because 112$

$\therefore 112 \ldots$

1) 114

i) 110.

11120

- $153 \%$,

1126 +

i) 112 का

$\because 11, \because 1$

41133

$\therefore 1.3$

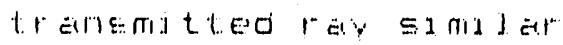

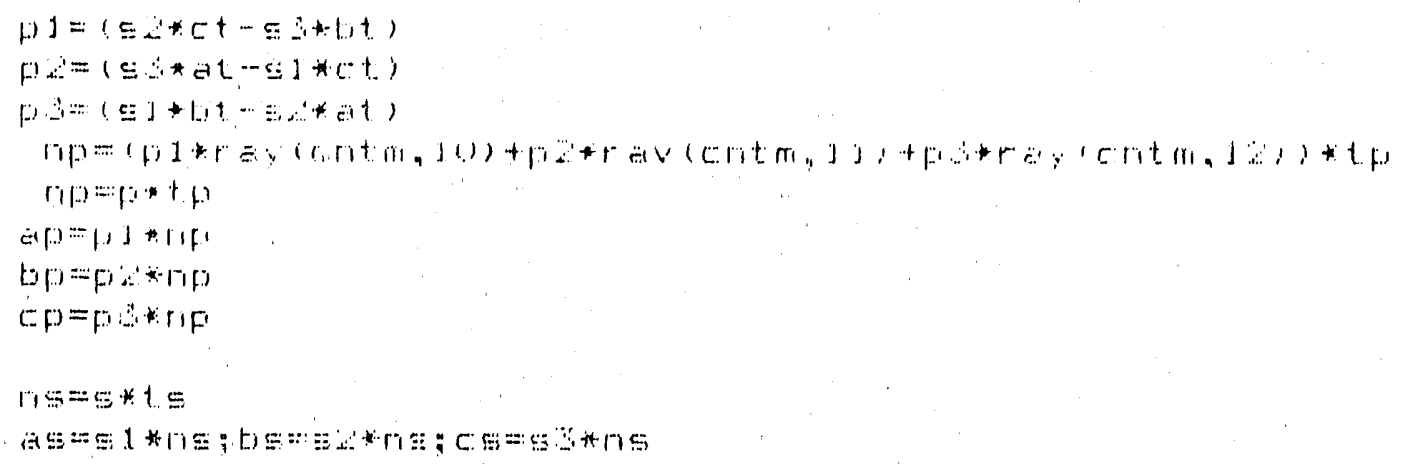




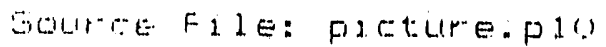

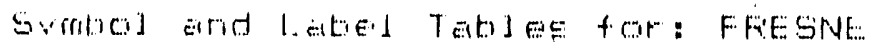

$\therefore$ intill

$\because 1,1.1$

$1+i$

a j 214

(1)

- $11=$

15

तn

$r \omega$

- $\quad F$

$\therefore 1$

(a)

i)

$\because$

$4=$

ㄷ.

a

lif

(i...:

…

iif

ri:

i1 t:

311

=․․

故

$\div 1$

Nii

19

it

i.t

t

i.

5

$1 \therefore$

$\therefore 1$

$\therefore$

$\therefore$

$\therefore$

- 1

$i \cdot 1$

$i$

- i.

$\therefore 1,1$

3111

1111

$1,1,11$
Y YFE

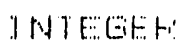

ritel.

Fit:Al

FVEAL.

FIEAIL...

ritefiL

NEAL

HEAlt.

Fit.:All.

1.EAI.

litent.

kit.int.

r.t.... Al...

rifint.

lifint.

Fil:.:int

FEE ril.

Fif

refint

PEAL.

FitE的.

bile fil..

HERL

1.

VIE FL.

leitil.

FEEHL

FiEGL

I. E. I L

fifist

rit. in.

irt.fil.

FiEfl.

ICE

1.1.1.

1. E. III...

Fit.int.

1.1.1

fit. rit.

lik...mil..

| if in

rit.int...

1.1.1.1.

|rial.

小

d 1 t
向LDFtes

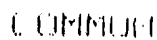

1.111. 117$)$

(4)

(1) (1) (1)

(1)0)1000)

borondend

0.1609060 .100

100)(0) 14

()

6111016) 4

प000100

(1)610610

0010001940

(1)

0.000101010

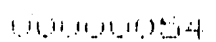

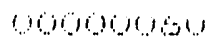

11000004

अ)(110)\%

(11)060) 4

1.101000100

acong d.4

1 10011!110

(4010101 1

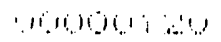

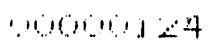

$11010101]$

다. 11010104

61100) 40

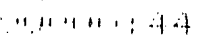

101101101501

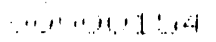

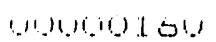

111111.1104

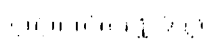

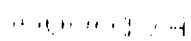

. 11.1111.1.11

‥11111. 614

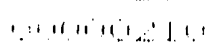

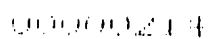

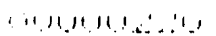

11111111). 4

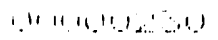

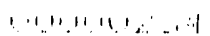

111111119.19491

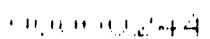

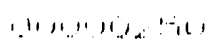

siltilit:

L. $1.11 .+1.1$

l.it. 1

FIt: PILIF:

$j i$

$1 ; 4.4:$ 


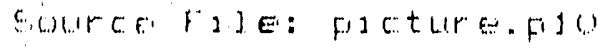

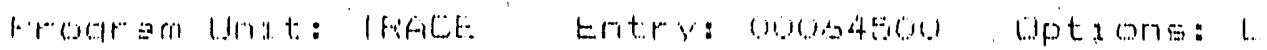

11] $1: 2:$

21194

(1) 1.35

11110

i) 1 . '?

111018

(1)

- 41.10

(1) 1141

() 1142

i) 49

- ⒈144

i) 1145

(1) 146

1..114\%

41149

$\because 114 \%$

11150

() 1191

1) 115

ㄱ. 115

(1) 154

(1) 115

) 11 10

\%11\%

) 115

त11

1.1 $1 \dot{0}^{11}$

1)1 131

(1) $16 \%$

1110

$\cdot 1164$

.1 18

$\because$ L i.......

$1196 \%$

11165

$\therefore 11 \div$

…

$\because: 1: 1$

11,2

$\because 11 !$ :

$111: 4$

$\because 11,6$

$111 \%$

- $\because 11 !$

.1 112

$\therefore 11 \%$

$.11 \mathrm{1} 80$

․ 181

$116 \ldots$

$\therefore 119$

1) 160

$\because 118$

$\because 116$

. 119

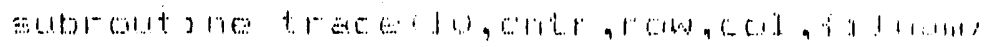

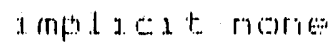

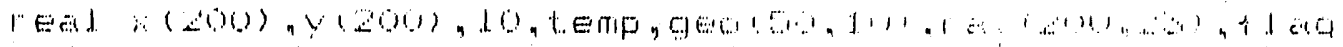

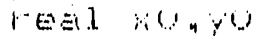

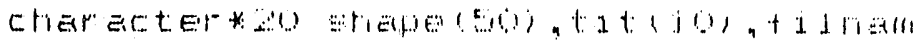

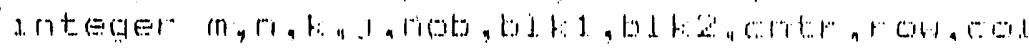

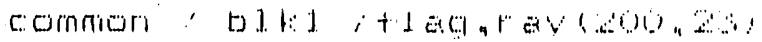

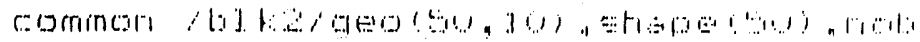

돌 $\operatorname{tat}$

$f: c n t r+1 ; n=2:-$

u(j) $=r$ a

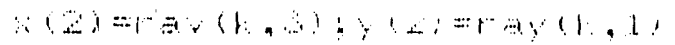

tit $(7)=[y] \in$

tit: $(t)=$, o17

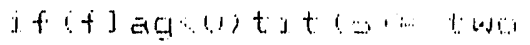

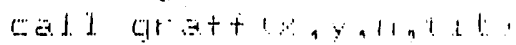

$r \in t a n$

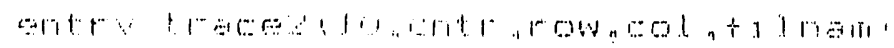

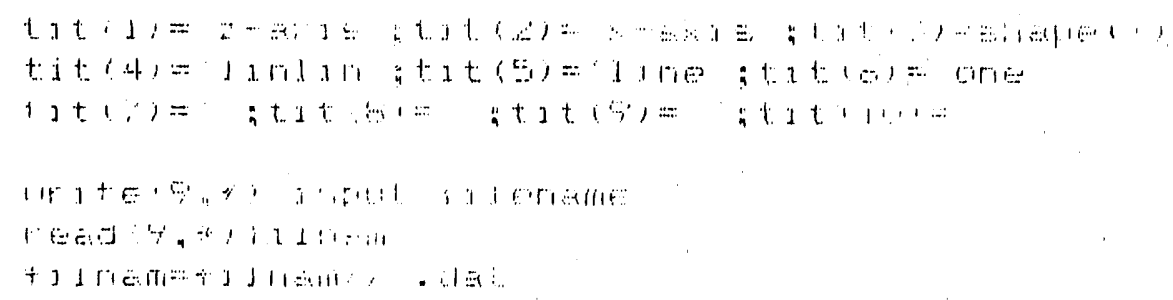




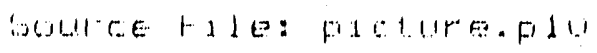

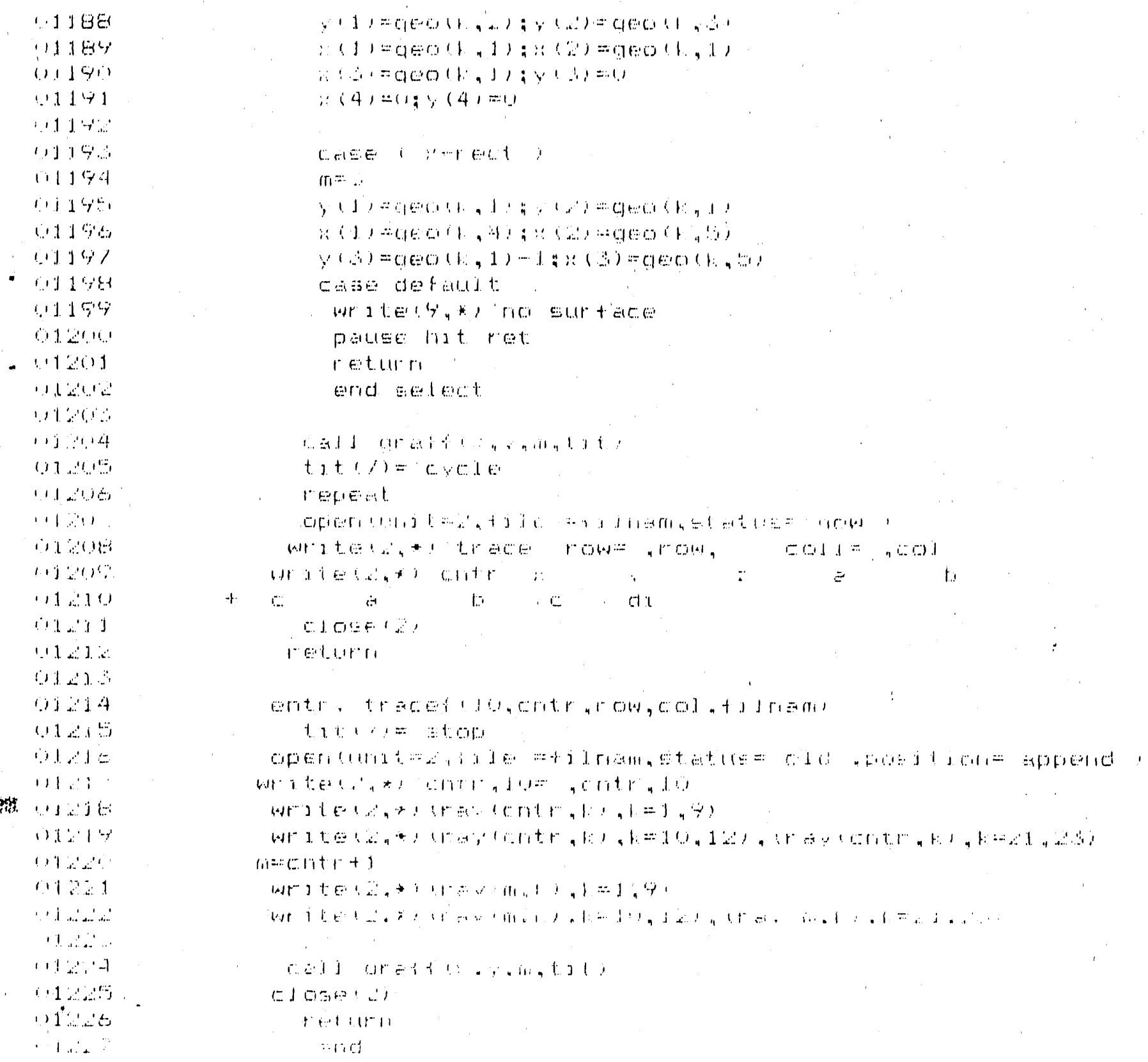




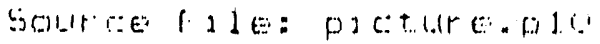

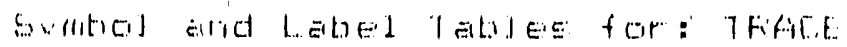

\section{$6 \times 11: 411$}

11.

0.1711

1.3 .1

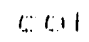

- $\quad$ t 1 1

$\therefore$

i

1.

$-400$

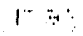

$\because 1=1$

$\therefore 4$

11

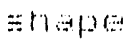

1 t.

in

11

1

1

1100

(i) $1: 1$

t. $1.1: .$.
TYF

lit: $+1 \mid$

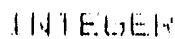

11. I E.BEtr

INI lizifiti

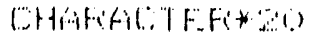

He: Fal.

ritil

Filion...

Pitzil.

liteit

feten

$|+| \cdot|-1|$

1.t. fill.

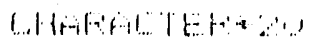

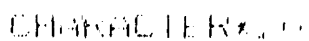

JATE TEF

J 11 tots

1WIELFH

I I I L C C HE

INTEGE:

1.NIE Cof

J)

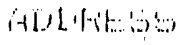

(.. $.1111111 . .114$

1.114. 19:

1.11.11117i

Uell lla;

[MJlM

$11.1111 \%$

(11.11(10) 16

(19)11.) ] ]

(11).11, 1..1)

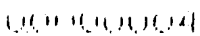

6016 1161010

1119.1419 .1104

$(11,114) 2.14$

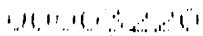

1010.19\%, 4

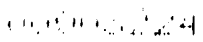

111111.1001

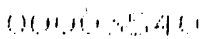

$410101040+4$

10101)

600064

(10)

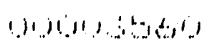

$1: 11$

1.i. 1. 1

L. I I I

1:11. f..

[1] 12
a.1 Thlibil.
In: $1181 \%$
1. $|| 4 .+1 \mid$.
L.LI I
liti:
100100
i. .

1. Heit:1

+1.

,

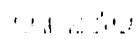

$.1+\ldots 1$

$11 \ldots$

- $1 . \therefore$

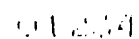

$111 . \cdots$,

- 11 . il,

$11 \therefore \therefore$

$111 \ldots$

$1119: \therefore$

1.1) $\therefore+11$

12.11

11.4 .2
1. filtit.

1.1911.1. 


\section{Appendix B}

trace row $=1 \quad \operatorname{col} 1=1014$
entr $x \quad y$
a
b
c:
$a$
b c
$\theta i$
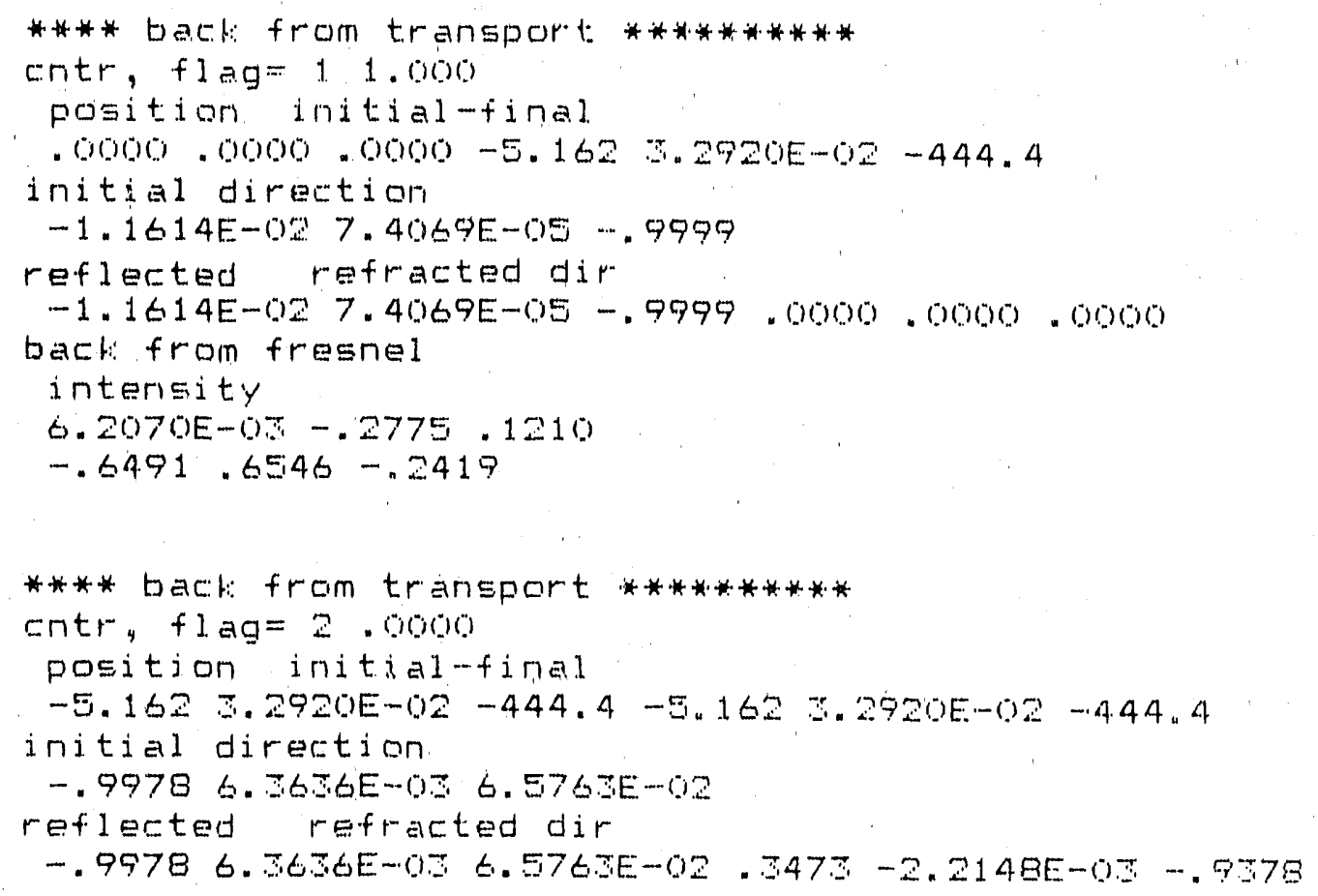

*** back from transport ****************

critr, $f 1 a g=\Xi 1,000$

positjon initial-fin̈l

-5. $162 \quad 3.2920 E-02-444.4$ …4.684 2.9872E-62 - 445.7

initial direction

. $3472-2.2148 E-0.5-.938$

reflected refracted dir

$.9475-2.2148 E-05-.9579$. 0000 . 0000 . 0000

back: from fresned

intensit' $r$

$-1.0779 E-02.5098-4.6755 E-02$

$-.6947 .57659 .50 .56-02$

\footnotetext{
** bacl: from transport

(:nter, fl. $a g=41.00(0)$

Fosition iritial-firal

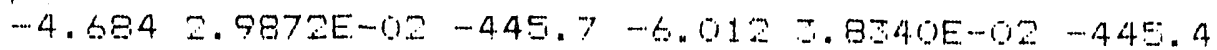

initial direction

$\cdots .9697$ t. $156 E-0.26 .22$

reflected refracted dir

-.9647 t. $1526 E-0 . .2632-.136 \theta 8.7214 E-04-.9906$

bacl: from fresnel

iriterisity

$-6.4571 E-0.1216-4.1399 E-0.5$

$6.5024 E-0.5$. $2849-4.7507 E-02$
} 
** bacli from transpart

cntr. +1 a $g=51.000$

position initial-final

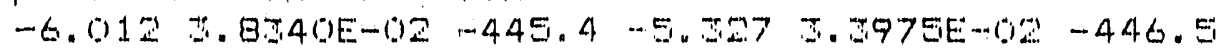

initial direction

$.497-\square .1714 E-1.2-.9676$

reflected refracted dir.

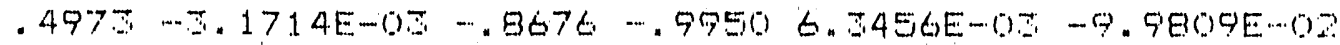

back: from frester.

intensity

I. $1103 E-0445.7871 E-02-5.7544 E-(.44$

$-7.6077 E-0.0 .1069-2.5725 E \cdots 04$
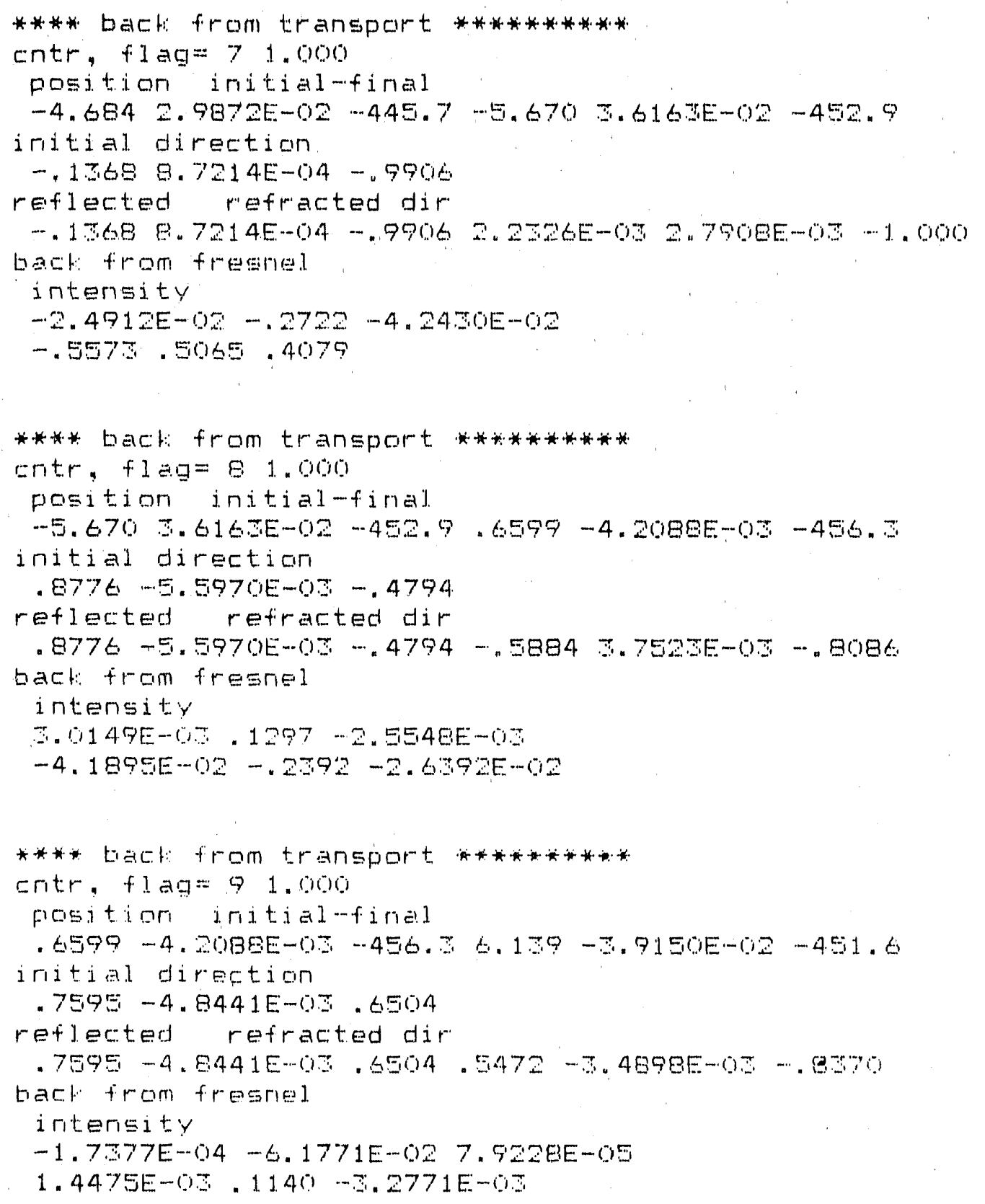
*** bactr from transport

cnt:r, $f 1 a g=11,0000$

POSition initial-final

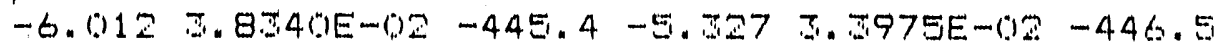

initial direction

$-.995060 .546 E-(0)-9.9809 E-02$

reflected refracted dir

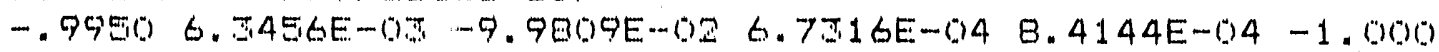
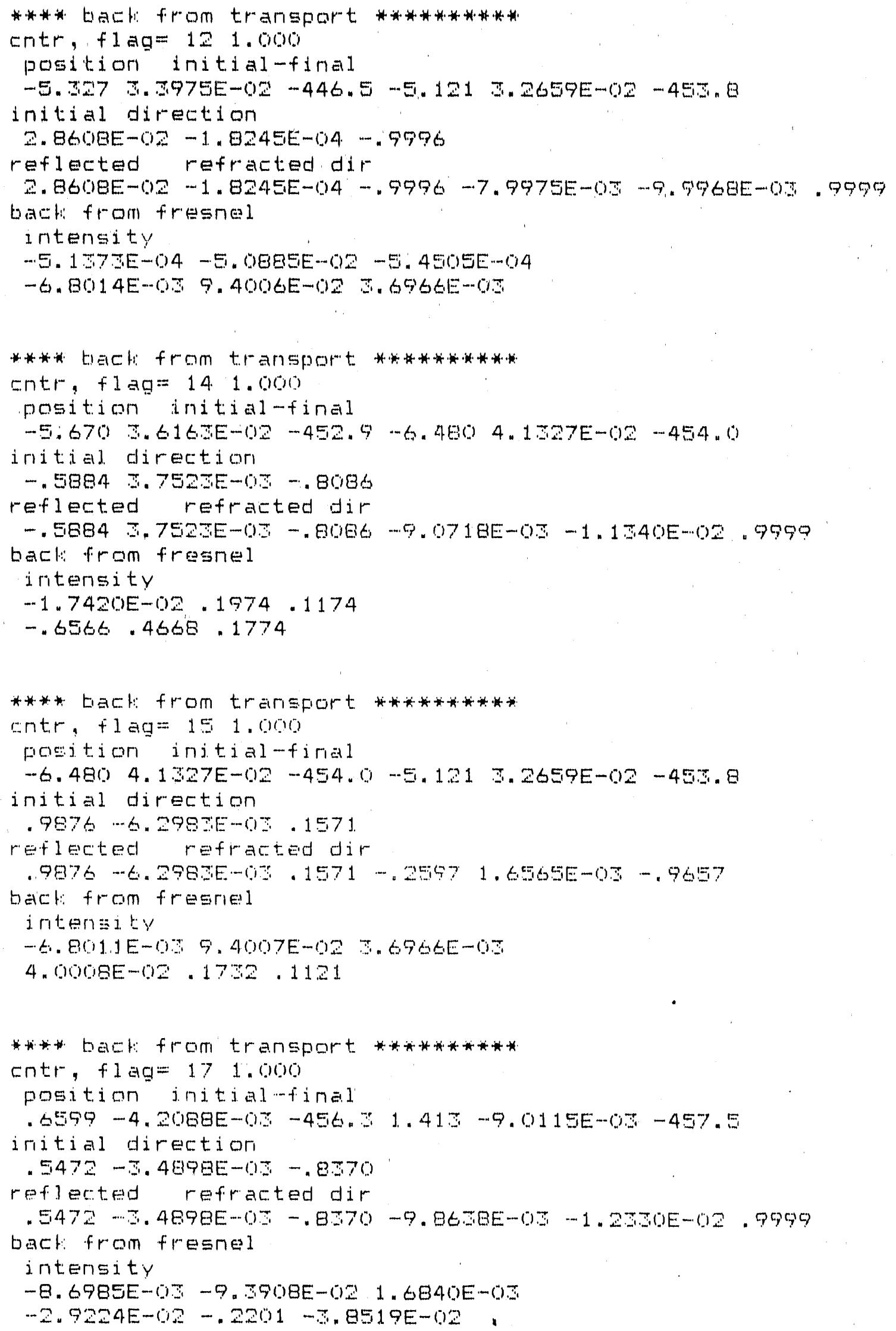
*** bacti from tramsport

contr, flag= 191 . $110 \%$

POEition initial-final

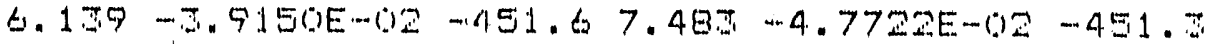

initial dimetion

$4767-6.220 日 E-1.2 .2 .46$

reflected refracted dir

$.9767-6.228950 .02 .2146 .9052-1.9355 E-02-.4207$

toack from fresmel

intensity

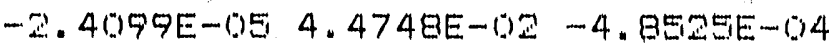

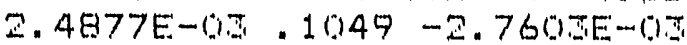

**** back from transport ***********

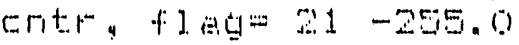

*******intensity=173.5

position initial-final

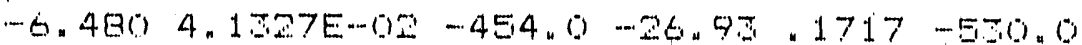

i ritial direction

-.2597 1.6565E-0.5. - . 9657

$r e f l e c t e d$ refracted of

-.2597 1.6565E-0.3 -.9657 .9958 .. $.1712 E-02-8.8292 E-02$

*** back from transport

critr, flag= 22 1.000

position initial-final

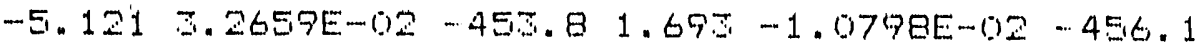

initial direction

$.9447-6.02476-05-.3279$

reflected refracted dir

$.9447-6.0247 E-0.5-.9275 .9915-2.1242 E-02-.1296$

back from fresnel

interis $v^{\prime}$

$-6.8548 E-0 Z-8.25 B 1 E-025.2962 E-07$

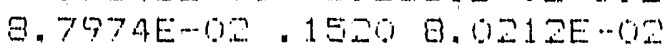

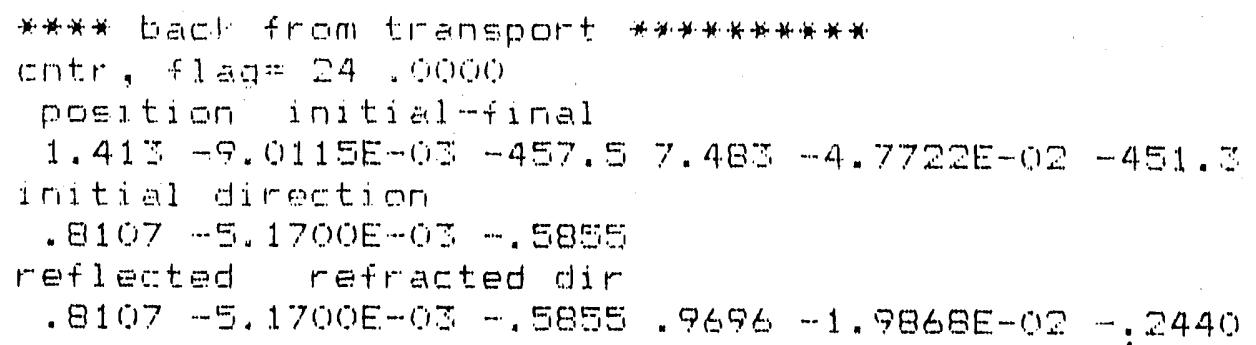

*** baci: from transport:

entr, flag= $25,(0)(0)$

fositian initial-firal

$7.48 .-4.772 .2 E-02-451.31 .693-1.0798 E-02-456.1$

initial direction

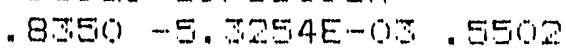

reflected refracted dir

$.8350-5.3254 E-0.5502-.1970 .3 .4050 E-0.4 \ldots .904$ 


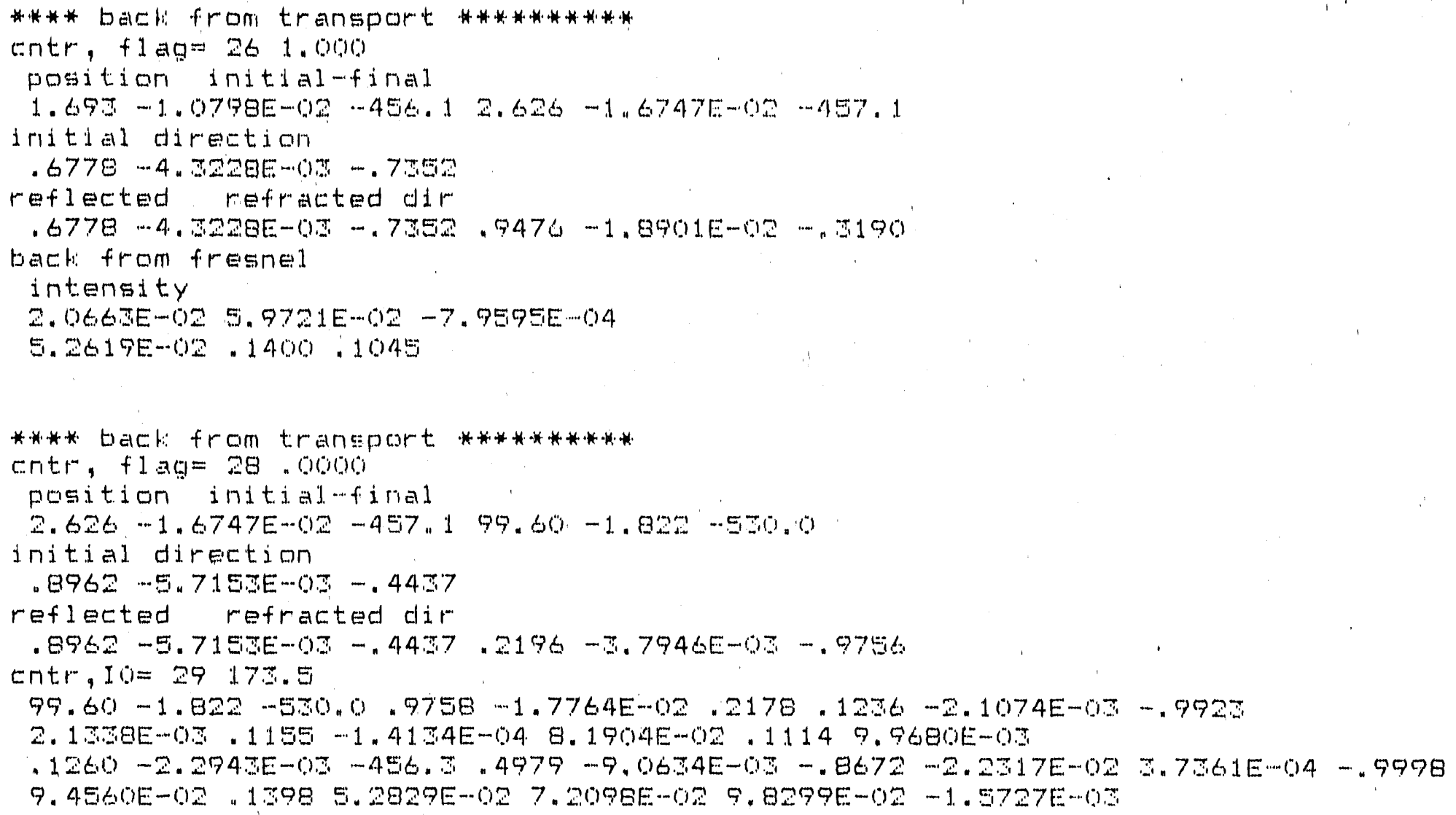




\section{Append IX B}

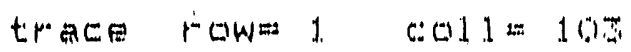

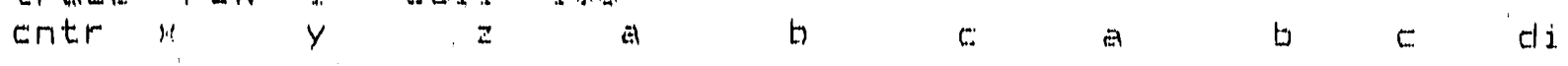

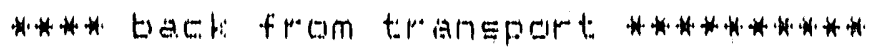

cotr, flatg= 1 , 1 , (ic)

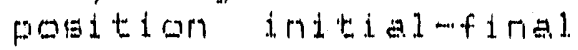

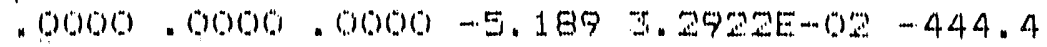

initial direction

$-1.1673 E-027.4069 E-05-.9599$

- reflected refreated dir.

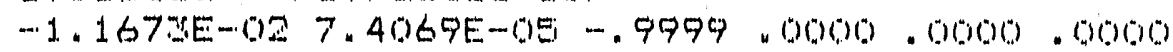

back: from fresner.

intensity

$4.97 \pm 1 E-63-.2766$. 1190

$-.6487 .6541-.2437$

**** backi from transport ***********

entr, $f .1 \mathrm{ag}=2 \cdot 0000$

position initial-final

-5.189 5.2922E-02 -444.4 -5.189 .

initial direction

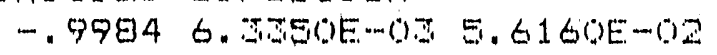

reflected refracted dir

-.9984 6. $9050 E-035.6160 E-02.349-2.2194 E-05-.956$

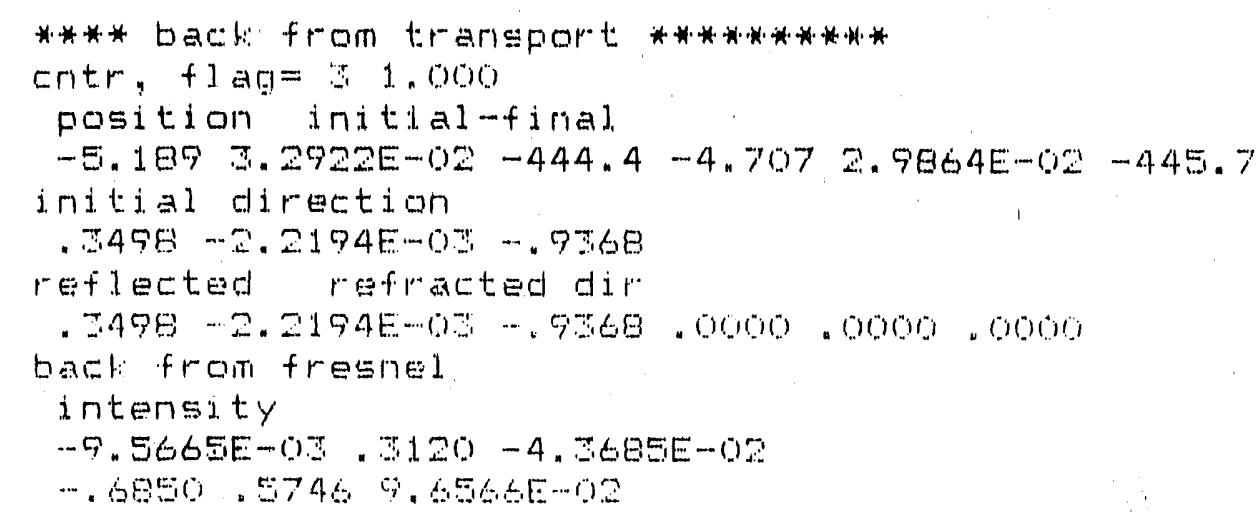

*** ta ack: from transpart

cintr, flag= 41 , 100

position initial-final

$-4.7072 .9864 E-62-445.7-6.059 \quad 5.8516 E-02 \quad .445 .4$

initial direction

-. . 9668 b. $1344 E-03$. 255

reflected refreated dir

-.9668 6.1344E-0. .2555. - . 389 6.8112E-04 - .9903

back: from fresnel

j. 11 tensity

-. $.8592 E-0.1229-3.9379 E-0.3$

6.7.5E-0. . $2866-4.4257 E-02$ 
* * ba back from trameport

entr, flagm $51.0 \%$

POE1tion indtial-intiol

-6.039 .

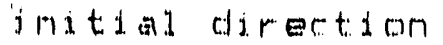

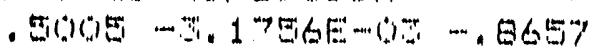

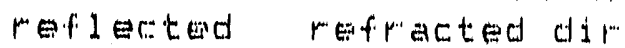

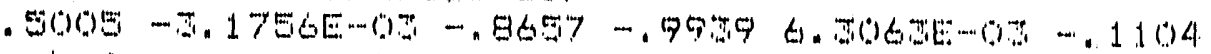

batel from fremal

1 1 tems 1 tir

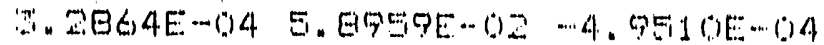

$-6.9655 E-(0), 1078-2.101040-104$

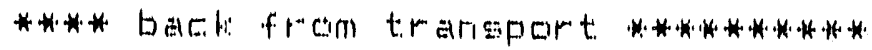

cnter, flact 71,000

pDEition indtial-final

$-4.7072 .9664 E-02-445.7-5.697 \quad 3.61518-0.2-472.6$

indtial direction

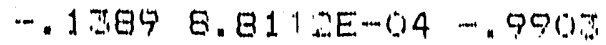

reflected refracted dip

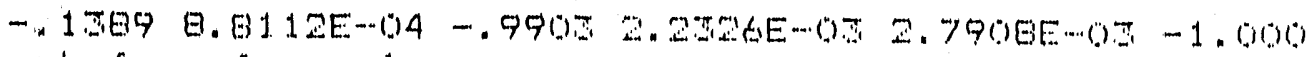

back from fresnel

lotemgity

-1. 4019E以O2 - . 27. -

$-.5551 .0010 .4122$
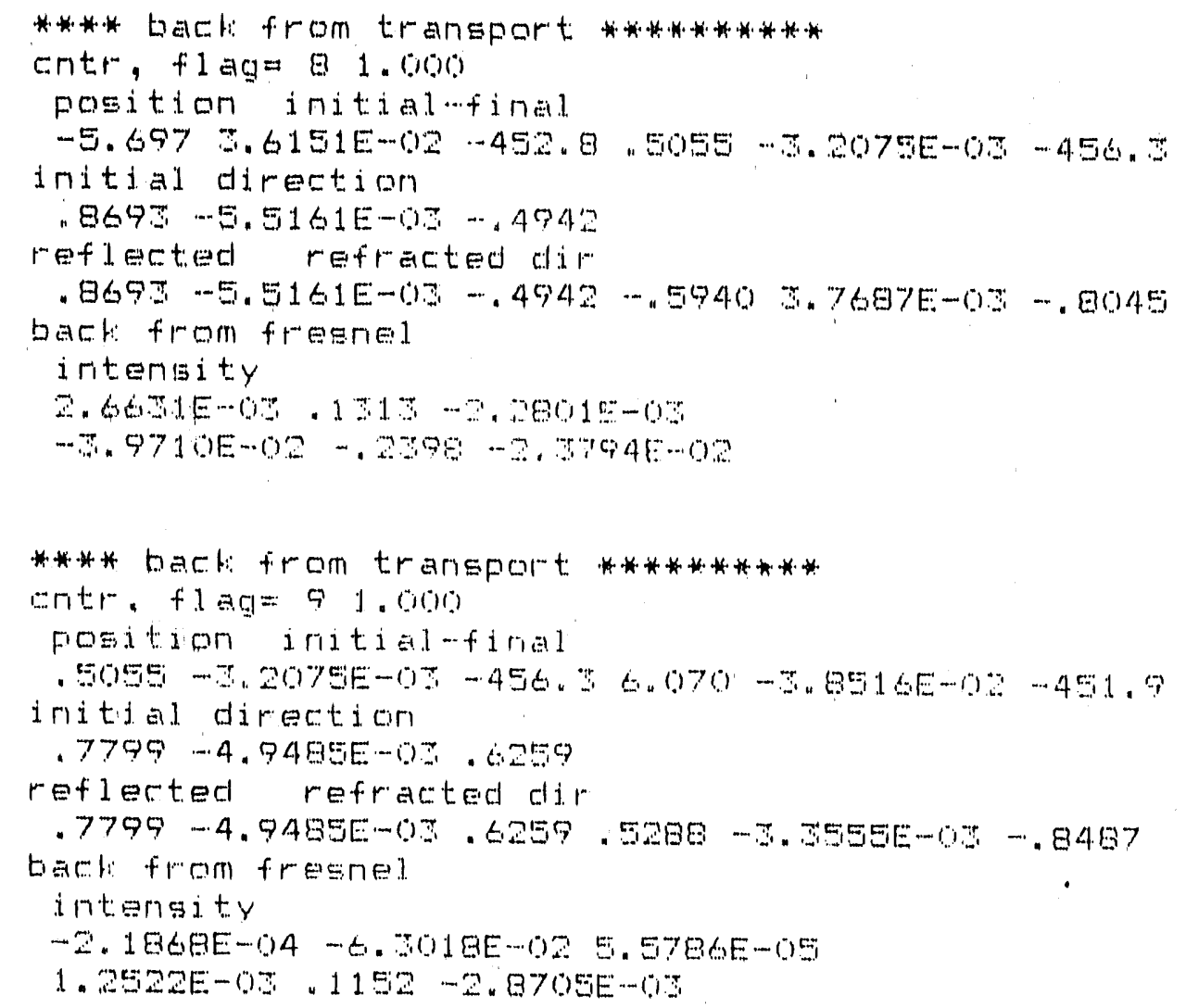
*** bac brom trmasport

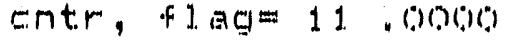

pogition 1mitiallatial

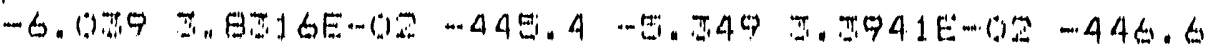

1 intial distion

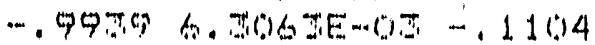

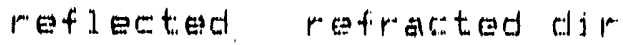

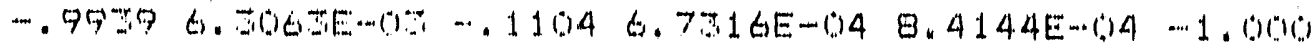

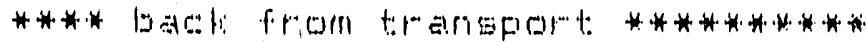

critr, f. $1 \mathrm{ag}=121.000$

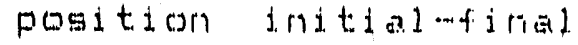

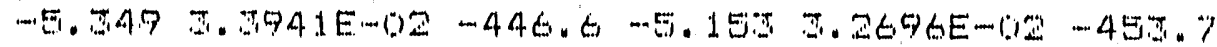

ind tial directidor

2. 7467E-(1) $-1,7441 E-(, 14 \quad \cdots .7996$

reflected refreated dir

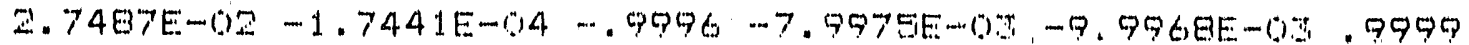

bati: trom frescies].

intensit'y'

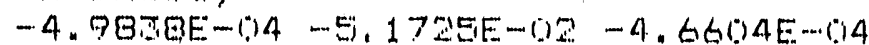

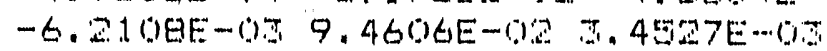

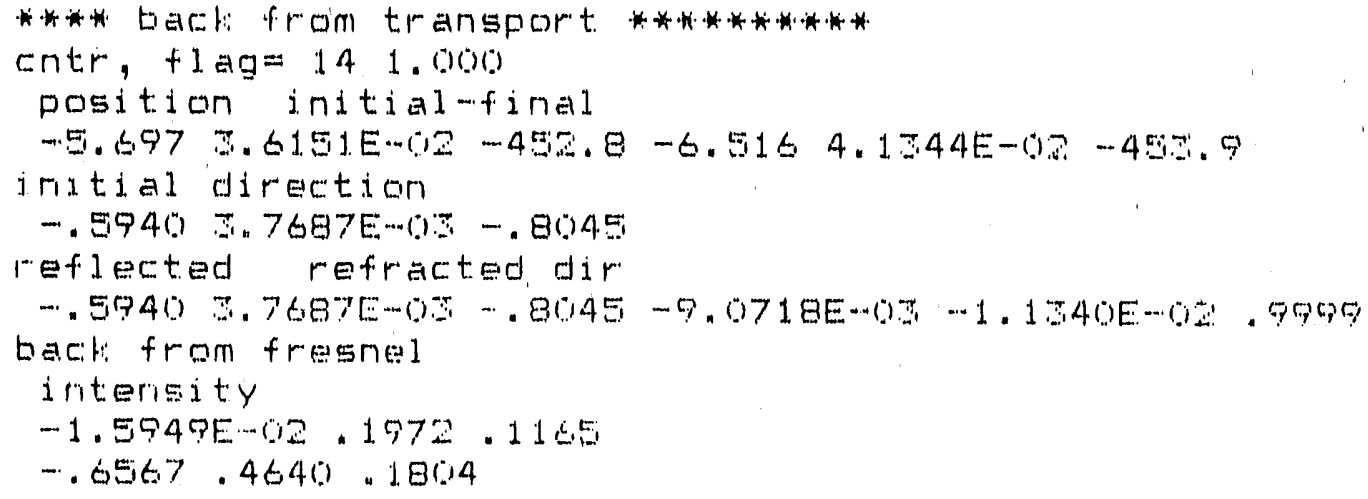

*** Le Let: from transprint

entr, $+100=15,1.0 \%$

position initial-ifiral

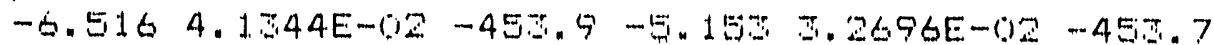

initial direction

$.9893-6.2770 \mathrm{a}-0.1460$

reflected refracted dir

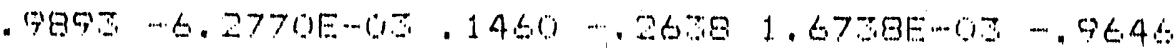

bach: from fresne]

intersity

-6.2112E-0I 9.4606E-02 I. 4528E-OB

$4.140 .10-1726 \cdot 1104$

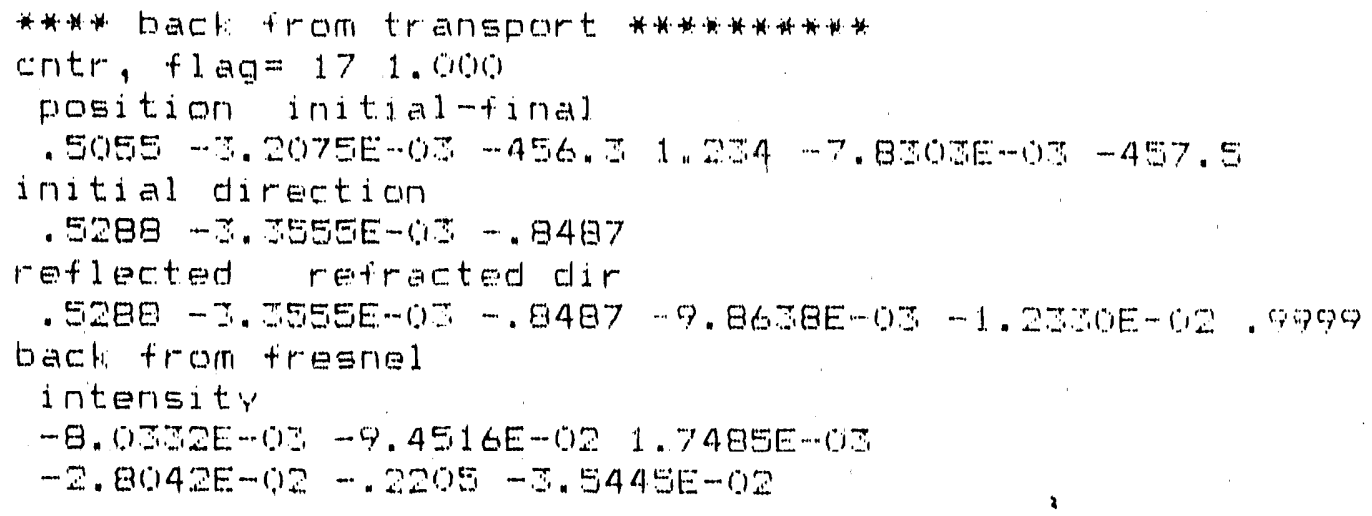




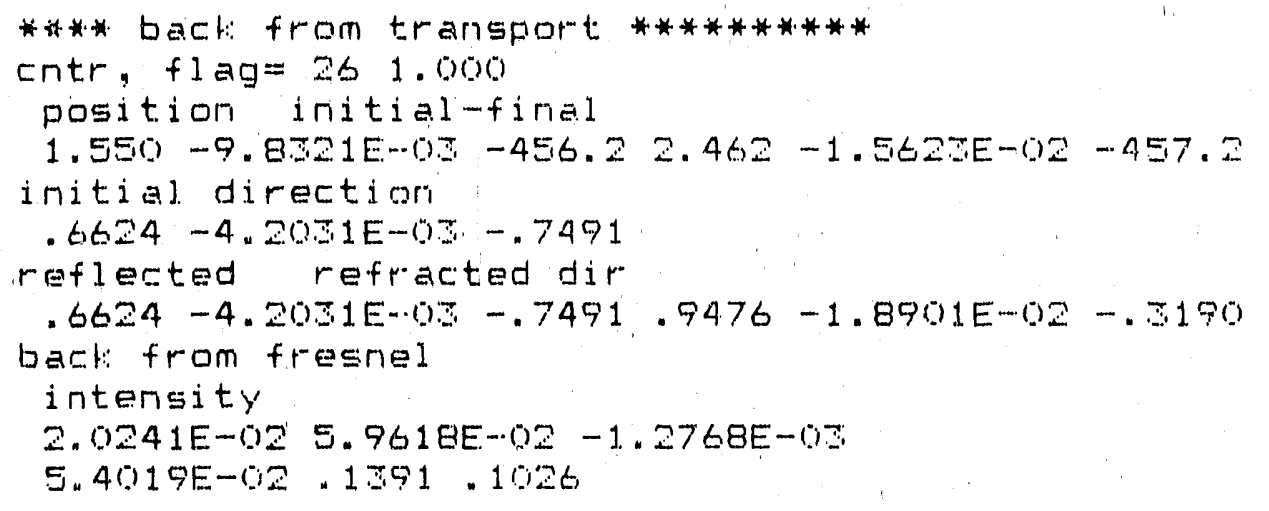

**** bact: from transport ***********

entr, flag= 29.0000

PQEition injtial-final

$2.462-1.5625 E-62-457.299 .60-1.822-5.0 .0$

initial direction

$.8880-5.6578-0.3-.4598$

reflected refracted dir

$.8880-5.6547-0.5-.4598 .2196-5.7946 E-10 .-9756$

Entr, IO $=29$ 18t. 1

$99.60-1.822-530.0 .9758-1.7764 E-02.2178 .1256-2.1074 E-03 \quad-9923$

2.1.38E-0.5.1155-1.41.4E-04 8.190,4E-02.1114 9.9680E-0.5

$.1260-2.294 .5 E-0.5-456.3 .4979-9.0654 E-0.5-.6672-2.2517 E-025.7261 E-04-.9998$

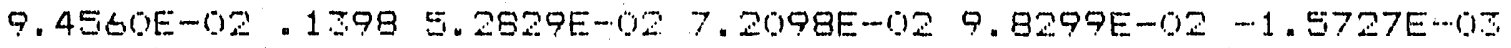



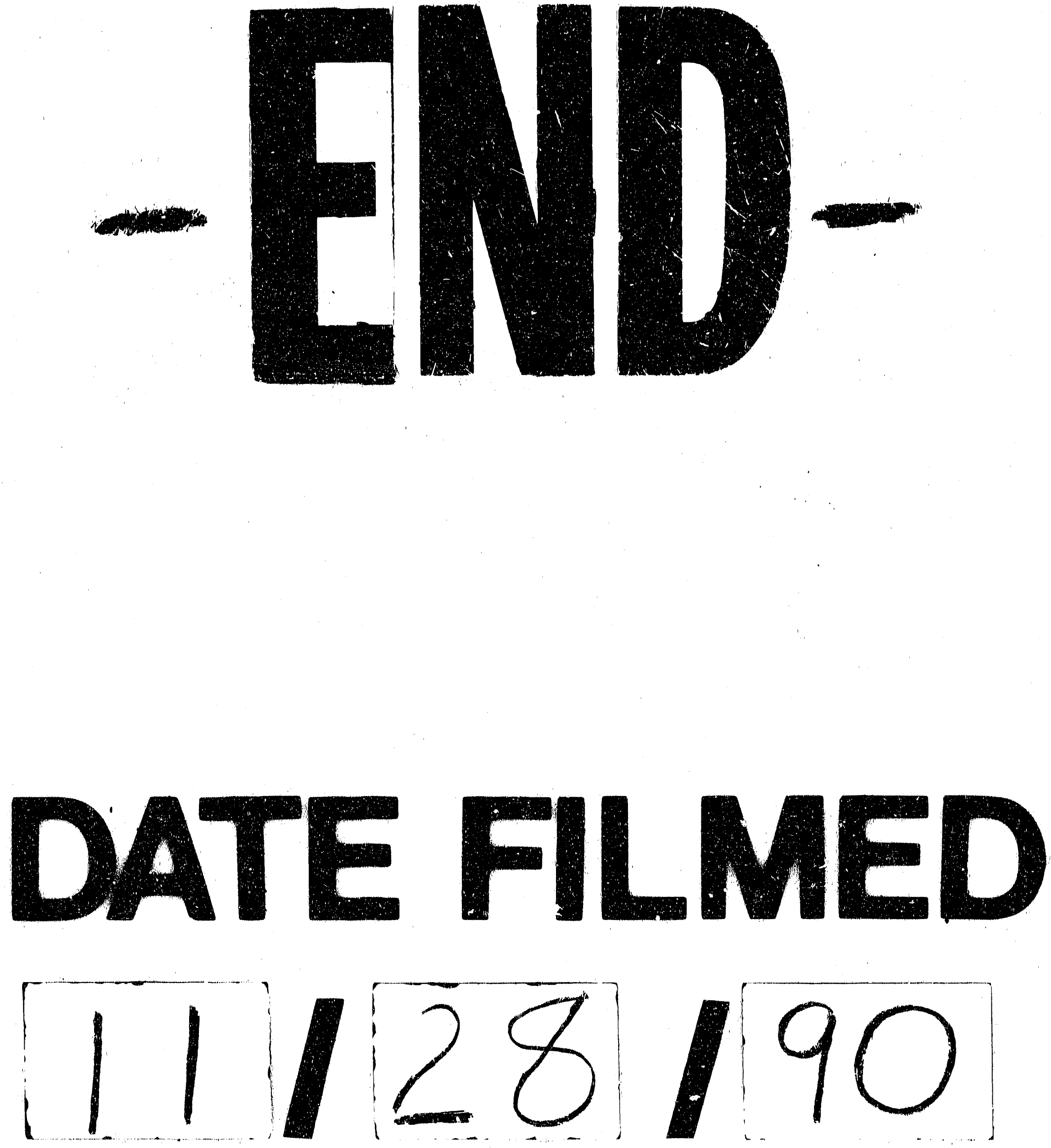
